Instituto DE FísicA DA Universidade DE SÃO PAULO

DissertaÇÃo DE MESTRADO

\title{
Aniquilação ressonante de pósitrons em gases moleculares
}

Autor:

César Augusto Nieto Acuña
Orientador:

Prof. Dr. Márcio Teixeira do

Nascimento Varella

Dissertação apresentada ao Instituto de Física da Universidade de São Paulo para a obtenção do título de Mestre em Física

IFUSP

São Paulo, SP, Brasil. Julho de 2014 
"As a boy Kepler had been captured by a vision of cosmic splendor, a harmony of the worlds which he thought so tirelessly all his life. Harmony in this world eluded him. His three laws of planetary motion represent, we now know, a real harmony of the worlds, but to Kepler they were only incidental to his quest for a cosmic system based on the Perfect Solids, a system which, it turns out, existed only in his mind. Yet from his work, we have found that scientific laws pervade all of nature, that the same rules apply on Earth as in the skies, that we can find a resonance, a harmony, between the way we think and the way the world works. When he found that his long cherished beliefs did not agree with the most precise observations, he accepted the uncomfortable facts, he preferred the hard truth to his dearest illusions. That is the heart of science."

Carl Sagan, The Harmony of the Worlds. Cosmos (1980) 


\begin{abstract}
The annihilation rates for slow positrons in molecules are enhanced for positrons with specific energies. According to the current view of the problem, such phenomenon corresponds to a positron continuum-discrete coupling, accompanied by a coupling of the molecule vibrational states. Such mechanism is described by a vibrational Feshbach resonance.

There are, basically, three developed topics in the time of the master degree. The first one is proposing an analytical model for the calculation of the coupling widths of the positron with Raman-Active vibrational transitions. It is showed that this estimation could be computed by some parameters of a single molecule and its order of magnitude is equivalent to coupling widths for IR-active transitions.

The second developed topic is the estimation of electron-positron correlation effects in the vibrational dynamics of the molecule mainly by defining couplings between modes which allows excitation of vibrational states, otherwise, inactive. As an example, the effects of the correlation in the $\mathrm{NaH}$ and $\mathrm{LiH}$ molecules have been presented.

An estimation of electronic structure and vibrational properties was also made for molecules with many vibrational normal modes. As an example, the HCN molecule at MP2 level calculation was taken, showing a method to approximate the electron-positron correlation effects, on the potential energy surface, at harmonic level, the electron-positron correlation effects on the potential energy surface.
\end{abstract}




\section{RESUMO}

As taxas de aniquilação de pósitrons lentos em moléculas são aprimoradas para pósitrons incidentes com energias específicas. Segundo a visão atual do problema, tal fenômeno corresponde a um acoplamento contínuo-discreto do pósitron acompanhado com transições vibracionais na molécula. Este mecanismo é conhecido como ressonância vibracional de Feshbach.

Existem basicamente três aspectos que foram desenvolvidos no tempo do mestrado. O primeiro foi a proposta de um modelo analítico para o cálculo das larguras de acoplamento do pósitron em transições vibracionais Raman-Ativas mostrando que sua estimativa pode ser feita a partir de parâmetros da molécula isolada e sua ordem de grandeza é equivalente ao das larguras associadas a transições IR-ativas.

O segundo aspecto desenvolvido é a estimativa dos efeitos de correlação elétron pósitron na dinâmica vibracional da molécula principalmente definindo acoplamentos entre modos vibracionais que permitiram a excitação de modos vibracionais, de outro modo, inativos. Foram apresentados, como exemplo, os efeitos de correlação nas moléculas de LiH e NaH.

Também foi feita uma estimativa das propriedades de estrutura eletrônica e vibracional para moléculas de mais de um modo normal de vibração. Especificamente, para a molécula de HCN no nível de cálculo MP2, apresentando um método para aproximar, no nível harmônico, os efeitos de correlação elétron-pósitron na superfície de energia potencial. 


\section{Agradecimentos}

A experiência de viver no Brasil foi muito importante para minha vida. Aqui encontrei um mundo diferente cheio de muitas boas pessoas e um nível académico excelente. Gostaria de agradecer a o Instituto de Física por confiar em mim e dar-me todos os espaços e ferramentas para culminar meus estudos de mestrado.

Dentro das pessoas que ajudaram no trabalho, agradeço de sobremaneira ao professor Márcio Varella pela sua orientação. Ele sempre esteve pronto para me ajudar em momentos complicados da minha estadia no Brasil e com a sua paciência, levar-me pelo caminho certo na pesquisa. Embora algumas vezes eu não concordava com ele, no final quase sempre descobria que ele tinha razão.

Ao professor Andrés Reyes e seu aluno, Jhonathan Romero do grupo de Química quântica da Universidad Nacional de Colombia pelos seus conselhos. Eu sempre admirarei seu trabalho em equipe e sua disciplina.

Obrigado aos meus amigos no Brasil: Carolina, Antonio, Cristian, Rômulo, Juan, Tatiana, Diana, Elkin e Ana Maria que me brindaram sua companhia durante estes dois anos. Em momentos difíceis, sempre contei com suas mãos, me apoiaram e acreditaram em mim, sempre contagiaram-me com sua dose de energia.

Agradeço a minha família pela sua ajuda e apoio . Foi difícil separar-me de eles mas acho que valeu muito a pena. Deixaram um Físico no aeroporto e receberam um mestre dois anos depois.

Obrigado a todo o pessoal do IFUSP, CAPES PROEX, CNPq e FAPESP pelo suporte financeiro. 


\section{Conteúdo}

$\begin{array}{lll}\text { Abstract } & \text { ii }\end{array}$

Resumo iii

Contents $\quad$ v

List of Figures viii

List of Tables $\quad$ ix

1 Descrição geral do trabalho $\quad 1$

1.1 Introdução . . . . . . . . . . . . . . . . . . . . . . . 1

1.2 Aspectos abordados no trabalho . . . . . . . . . . . . . . . . . 4

1.3 Organização do texto . . . . . . . . . . . . . . . . . 5

2 Introdução ao espalhamento ressonante $\quad 6$

2.1 Descrição experimental do problema . . . . . . . . . . . . . . . 6

2.2 Descrição da teoria de espalhamento ressonante . . . . . . . . . . . . . . 7

2.2 .1 Operador de transição $T \ldots \ldots \ldots$. . . . . . . . . . 8

2.2.2 Acoplamento entre um estado discreto e estados do contínuo . . . . 9

2.3 Descrição da excitação de níveis discretos do alvo . . . . . . . . . . . . . . 13

2.4 Operador de transição em um espalhamento ressonante incluindo transições dos níveis do alvo . . . . . . . . . . . . . . . . . . . . . . . 14

3 Graus de liberdade vibracionais da molécula $\quad \mathbf{1 5}$

3.1 A superfície de energia potencial . . . . . . . . . . . . . . . 15

3.2 Vibrações moleculares . . . . . . . . . . . . . . . . . . . . 16

3.2.1 Transições vibracionais IR-ativas . . . . . . . . . . . . . . . . 17

3.2.2 Transições vibracionais Raman-ativas . . . . . . . . . . . . . . . . 19

4 Formalismo de operadores de projeção de Feshbach e modelo de Gribakin-

Lee

4.1 Introdução . . . . . . . . . . . . . . . . . . . . . . . . 22

4.2 Formalismo de operadores de projeção de Feshbach . . . . . . . . . . . . 23

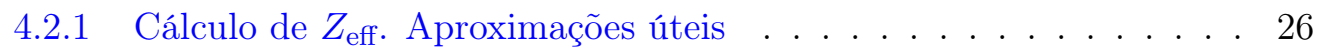

4.3 Modelo de Gribakin-Lee . . . . . . . . . . . . . . . . . . . . . . . . . . 28 
5 O acoplamento pósitron-molécula para vibrações com atividade Raman 30

5.1 Antecedentes . . . . . . . . . . . . . . . . . 30

5.2 Detalhes do modelo . . . . . . . . . . . . . . . . . 33

5.2 .1 Cálculo da amplitude de captura . . . . . . . . . . . . . . . 33

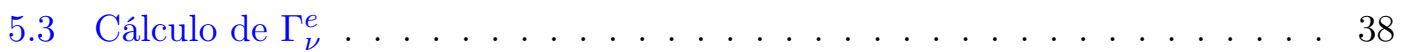

5.4 Conclusões . . . . . . . . . . . . . . . . . . . . . . . . 41

6 Acoplamentos vibracionais mediados pela correlação elétron-pósitron. 42

6.1 Introdução . . . . . . . . . . . . . . . . . . . . . . . . . 42

6.2 Métodos computacionais para o cálculo de estrutura eletrônica com pósitrons 43

6.3 Cálculos de curvas de energia potencial para moléculas diatômicas . . . . 44

6.4 Processo de Acoplamentos vibracionais . . . . . . . . . . . . . . . . . . . 47

6.4.1 Correlação pósitron-elétron como um potencial . . . . . . . . . . . 47

6.4 .2 Mecanismo de transição . . . . . . . . . . . . . . . . . . . . . . . . . . . . . 48

6.4 .3 Afinidade positrônica . . . . . . . . . . . . . . 50

6.4.4 Transições permitidas . . . . . . . . . . . . . . . . . 51

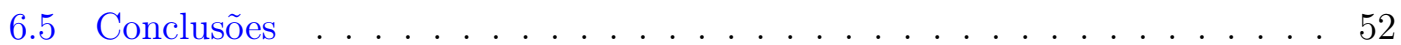

7 Acoplamentos vibracionais intermediados pela correlação elétron-pósitron para moléculas com mais de um modo normal. $\quad 53$

7.1 Introdução . . . . . . . . . . . . . . . . . . . . . . . 53

7.2 Mecanismo de transição para mais de um modo normal . . . . . . . . . . . . 54

7.3 Aproximação harmónica . . . . . . . . . . . . . . . . . . . 55

7.3.1 Construção da base de auto-estados do composto . . . . . . . . . 56

7.4 Aplicação da teoria à molécula de $\mathrm{HCN}$. . . . . . . . . . . . . . . . 58

7.5 Conclusões . . . . . . . . . . . . . . . . . . . . 63

8 Conclusões gerais e perspectivas futuras. $\quad 64$

8.1 Perspectivas . . . . . . . . . . . . . . . . . 65

$\begin{array}{lr}\text { A Cálculo de estrutura eletrônica } & \mathbf{6 6}\end{array}$

A.1 Método Hartree-Fock . . . . . . . . . . . . . . . . . . . . 66

A.1.1 Funções base . . . . . . . . . . . . . . . . . . . . . . 68

A.2 Correlação eletrônica . . . . . . . . . . . . . . . . . . . . 70

A.2.1 Funções de configuração de estado . . . . . . . . . . . . . . . . . . . . . 70

A.3 Teoria de Configuração de Interação (CI) . . . . . . . . . . . . . . . . . . 71

A.3.1 Teoria de perturbações de Molle-Plesset . . . . . . . . . . . . . 72

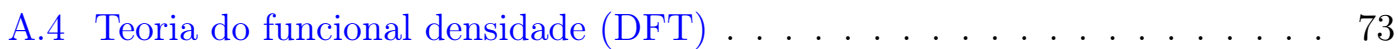

A.4.1 Funcionais troca-correlação . . . . . . . . . . . . . . . . . 74

$\begin{array}{lll}\text { B Aspectos teóricos da espectrometria vibracional } & \mathbf{7 6}\end{array}$

B.1 Análise vibracional . . . . . . . . . . . . . . . . . . . . . 76

B.1.1 Separação de vibrações e rotações. . . . . . . . . . . . . . . . . . . . . . . . . 76

B.1.2 Cálculo de auto-valores vibracionais . . . . . . . . . . . . . . 78

B.1.3 Deslocamentos cartesianos e massa reduzida . . . . . . . . . . . 78

B.2 Potencial de Morse . . . . . . . . . . . . . . . . . . . . . . . . . 79

B.3 Coeficiente de Einstein . . . . . . . . . . . . . . . . . . . 80 
B.4 Intensidade de Absorção infravermelha . . . . . . . . . . . . . . . . . . . . 81

C Aspectos formais do modelo de Gribakin-lee $\quad 83$

C.1 Aniquilação do pósitron . . . . . . . . . . . . . . . . . . . . . . . . . . . . . . . . . 83

C.2 modelo de Gribakin-Lee . . . . . . . . . . . . . . . . . . . . . . 84

C.3 Transformação de $Z_{\text {eff }}$ para comparar os resultados experimentais. . . . . . 85

Bibliografia 


\section{Lista de Figuras}

1.1 Deslocamento dos picos de $Z_{\text {eff }} \ldots \ldots \ldots \ldots$

2.1 Representação esquemática do processo de espalhamento . . . . . . . . . . 7

2.2 Ressonância . . . . . . . . . . . . . . . . . . . . . . . . . . 12

3.1 Espectro Raman . . . . . . . . . . . . . . . . . . . . 20

4.1 Ressonância vibracional de Feshbach . . . . . . . . . . . . . . . . . . . 22

4.2 Ressonância vibracional de Feshbach . . . . . . . . . . . . . . . . . . 28

5.1 (a) VFR no etileno, (b) Ajuste $\varepsilon_{b}$ vs $12.4\left(\alpha+16 \mu+2.4 N_{\pi}-5.6\right)(\mathrm{meV}) \quad$. 31

5.2 (a) Comparação entre a energia de ligação do modelo variacional e o numérico. (b) FUnções de onda $>$ Variacional e numérica . . . . . . . . . . . 34

5.3 (A) Extrapolação do parâmetro variacional (B) função de onda variacional

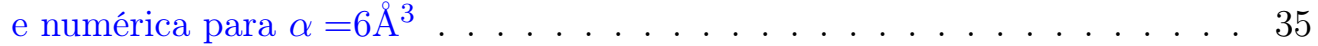

5.4 (A) Comparação do modelo variacional e numérico: $\left|A_{k}\right|^{2}$ vs A energia da transição vibracional para algumas polarizabilidades. (B) $\left|A_{k}\right|^{2}$ vs a polarizabilidade $(\alpha)$ da molécula para algumas energias de transição. . . . 37

$5.5 \Gamma_{\nu}^{e}(\mathrm{meV})$ para algumas transições Raman-ativas em função de $k_{\nu}\left|\alpha_{\nu}\right|^{2}$ para quatro moléculas. . . . . . . . . . . . . . . . . 40 40

6.1 Curvas de energia potencial para a molécula de $\mathrm{LiH}$ e $e^{+} \mathrm{LiH}(\mathrm{A})$ No nível MRD-CI (B) No nível UHF-MP2 . . . . . . . . . . . . . . . . . 45

6.2 Curvas de energia potencial para a molécula de $\mathrm{NaH}$ e $e^{+} \mathrm{NaH}(\mathrm{A}) \mathrm{No}$ nível MRD-CI (B) No nível UHF-MP2 . . . . . . . . . . . . . . . . 45

7.1 (a)Modos normais do HCN (b)Densidade de probabilidade do pósitron. . . 59

7.2 CEP para os modos longitudinais da molecula $\mathrm{HCN}$ e o composto $e^{+} \mathrm{HCN} \quad 60$

7.3 CEP para o modo transversal da molecula $\mathrm{HCN}$ e o composto $e^{+} \mathrm{HCN}$. . 60

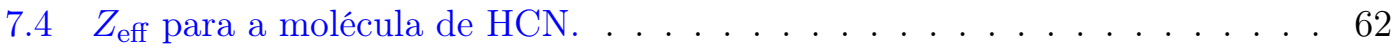

B.1 Potencial de Morse (unidades arbitrarias) e as densidades de probabilidade de suas auto-funções $\Phi_{0}$ (vermelho) e $\Phi_{3}$ (azul) . . . . . . . . . . . . . 79 


\section{Lista de Tabelas}

5.1 Larguras de ressonãncia para o Etano . . . . . . . . . . . . . . . 38

5.2 Larguras de ressonância para o Etileno e o Acetileno. . . . . . . . . . . . . 39

$6.1\left(\mathrm{E}_{0}\right),\left(R_{0}\right), \omega_{0}$ e termos anarmônicos para o $\mathrm{LiH}$ e e+ $+\mathrm{LiH}$ e $\mathrm{NaH}$ e e $+\mathrm{NaH} \quad 46$

$6.2\left|C_{\mu_{\nu}, 1}\right|^{2}=\left|\left\langle\mu_{\nu} \mid 1\right\rangle\right|^{2}$ para diferentes estados finais $\left(\mu_{\nu}\right) \ldots \ldots \ldots . \ldots . . \ldots 9$

6.3 Afinidade positrônica para as moléculas de $\mathrm{LiH}$ e $\mathrm{NaH}$. . . . . . . . . . 51

7.1 Parâmetros da dinâmica vibracional da molécula e do composto, onde $\omega_{k}^{\prime}=\omega_{k} \sqrt{1+\epsilon_{k}}, \epsilon_{k}=\frac{K_{k k}}{\omega_{k}^{2} m_{k}}, \Omega_{k}=F_{k} \sqrt{\frac{1}{2 m_{k} \omega_{k}}} \mathrm{e} \omega_{k, l}=\frac{K_{k l}}{2\left(\omega_{k} \omega_{l} m_{k} m_{l}\right)^{1 / 2}} . .61$

C.1 Modos vibracionais para a molécula $\mathrm{CH}_{3} \mathrm{Cl}$ e o fator de largura $\omega_{\nu} \mu_{\nu}^{2}$ que esta associado à largura eletrônica pela equação (C.7) . . . . . . . . . . 85 
Dedicado a você, a pessoa quem está lendo. Obrigado por valorizar meu trabalho. 


\section{Capítulo 1}

\section{Descrição geral do trabalho}

\subsection{Introdução}

O propósito deste trabalho é estudar, teoricamente, as taxas de aniquilação de pósitrons de baixa energia $(\lesssim 1 \mathrm{eV})$ em moléculas vibracionalmente ativas. Os pósitrons, antipartículas dos elétrons, são muito importantes em muitas áreas da ciência e da tecnologia. A sua utilidade vem do fato de que, toda vez que um elétron e um pósitron interagem, eles podem aniquilar-se produzindo dois ou três raios Gamma cada um com energia de várias centenas de $\mathrm{KeV}$ no referencial do centro de massa do sistema.[1]

Em ciência de materiais, há numerosas técnicas baseadas na aniquilação de pósitrons para estudar propriedades da matéria [2-4] incluindo superfícies de Fermi em metais [5] poros microscópicos em sólidos [6] e a composição e estrutura de superfícies [7]. Em medicina, a tomografia de emissão de pósitrons (PET) tira partido da aniquilação no estudo de processos de metabolismo humano [8].

No regime de energia no qual vai estar focado o trabalho $(\lesssim 1 \mathrm{eV})$, é conhecido que a aniquilação ocorre como resultado da simples sobreposição das densidades de probabilidade do elétron e do pósitron durante a colisão[1]. Matematicamente é calculada a taxa de aniquilação $(\Gamma)$ a partir da fórmula[9]:

$$
\Gamma=\pi r_{0}^{2} c n \sum_{j=1}^{Z}\left\langle\Psi\left(\mathbf{r}_{1}, \ldots, \mathbf{r}_{Z}, \mathbf{r}_{p}\right)\left|\delta\left(\mathbf{r}_{j}-\mathbf{r}_{p}\right)\right| \Psi\left(\mathbf{r}_{1}, \ldots, \mathbf{r}_{Z}, \mathbf{r}_{p}\right)\right\rangle
$$

obtida a partir da função de onda $\Psi\left(\mathbf{r}_{1}, \ldots, \mathbf{r}_{Z}, \mathbf{r}_{p}\right)$ que descreve um gás de $n$ moléculas por unidade de volume, cada uma delas com $Z$ elétrons descritos pelas coordenadas $\mathbf{r}_{1}, \ldots, \mathbf{r}_{Z}$ além da coordenada do pósitron $\mathbf{r}_{p}$. $r_{0}$ é o raio clássico do elétron (em unidades 
cgs, $\left.r_{0}=e^{2} / m c^{2}\right)$. A distribuição $\delta\left(\mathbf{r}_{j}-\mathbf{r}_{p}\right)$, é incluida para calcular a densidade do pósitron nas coordenadas dos elétrons.

Experimentalmente, $\Gamma$ é obtida a partir da quantidade de eventos de aniquilação em função do tempo. Toda vez que ela vai depender do parámetro experimental $(n)$, usualmente é definida a taxa de referência $\left(\Gamma_{D}\right)$ para um gás de elétrons livres com a mesma densidade de elétrons $\left(n_{e}\right)$. Ela pode ser estimada ignorando a correlação elétron-pósitron de maneira que suas funções de onda podem ser simples ondas planas na chamada aproximação de Born:

$$
\Psi\left(\mathbf{r}_{1}, \ldots, \mathbf{r}_{Z}, \mathbf{r}_{p}\right)=\exp \left(i \mathbf{k}_{1} \cdot \mathbf{r}_{1}+\ldots+\mathbf{k}_{z} \cdot \mathbf{r}_{Z}\right)
$$

O primeiro cálculo utilizando esse tipo de função de onda foi feito por Dirac [10] no caso que a aniquilação produz dois raios gamma, obtendo: ${ }^{1}$

$$
\Gamma_{D}=\pi r_{0}^{2} c n_{e}
$$

válida para o limite não relativista. Nos experimentos de aniquilação, de pósitrons em gases moleculares, foi observado que $\Gamma_{D}$ prevê resultados menores do que o esperado, de maneira que foi definido o parâmetro adimensional $Z_{\text {eff }}[11]$ pelo quociente:

$$
Z_{\text {eff }} \equiv \frac{\Gamma}{\Gamma_{D}}=\frac{\Gamma}{\pi r_{0}^{2} c n}
$$

onde $n$ é a densidade de átomos ou moléculas por unidade de volume.

$Z_{\text {eff }}$, usualmente é entendido, de maneira qualitativa, como o número efetivo de elétrons que contribuem na aniquilação. Se não são considerados os graus de liberdade internos da molécula, poderia ser esperado que $Z_{\text {eff }}$ fosse proporcional ao número total de elétrons por átomo ou molécula $\left(Z=n_{e} / n\right)$. No entanto, a primeira geração de experimentos, consistentes na simples mixtura de um gas de pósitrons com o gás de moléculas, realizados em armadilhas termalizadas à temperatura ambiente, foi encontrado que para algumas moléculas este $Z_{\text {eff }}$ medido em amostras a temperatura ambiente, pode ser muito maior do que o número de elétrons (por exemplo, para o butano $Z_{\text {eff }} / Z \approx 600$ )[12]. Aliás, o estudo de uma grande quantidade de espécies de moléculas incluindo líquidos de baixa pressão de vapor e sólidos permite encontrar que para moléculas maiores como o Naftaleno ou o Hexadecano, $Z_{\text {eff }}$ pode crescer ainda mais apresentando valores de $Z_{\text {eff }} / Z>10^{4}$.[13]

As primeiras tentativas desenvolvidas para obter uma teoria que explicasse esse crescimento nas taxas de aniquilação incluíram a hipótese de uma aniquilação aumentada

\footnotetext{
${ }^{1}$ Para esse caso, o momentum angular do sistema é nulo e o pósitron e o elétron encontram-se com spins contrários que é o caso mais provável.
} 
devido à existência de um estado ligado (ou virtual) com uma energia de ligação muito próxima a zero[14]. Os graus de liberdade vibracionais da molécula só começariam a ser tidos em conta como responsáveis pelos grandes valores de $Z_{\text {eff }}$, desde anos 70 's.[15]. No entanto, o progresso foi impedido pela falta de dados para pósitrons com valores de energia específicos[16].

No ano 1997, foram desenvolvidos feixes de pósitrons aproximadamente mono-energéticos ( $40 \mathrm{meV}$ FWHM)[17] permitindo medições de taxas de aniquilação em átomos e moléculas, para pósitrons incidentes com energias desde $50 \mathrm{meV}$ até alguns elétronVolts. Os resultados obtidos, permitem constroir graficos d $Z_{\text {eff }}$ em função da energia de incidência do pósitron. Eles apresentam picos localizados em energias similares às energias de transição vibracional da molécula isolada mas distribuindo-se ligeiramente deslocados desses últimos. da maneira apresentada na figura 1.1.[41].

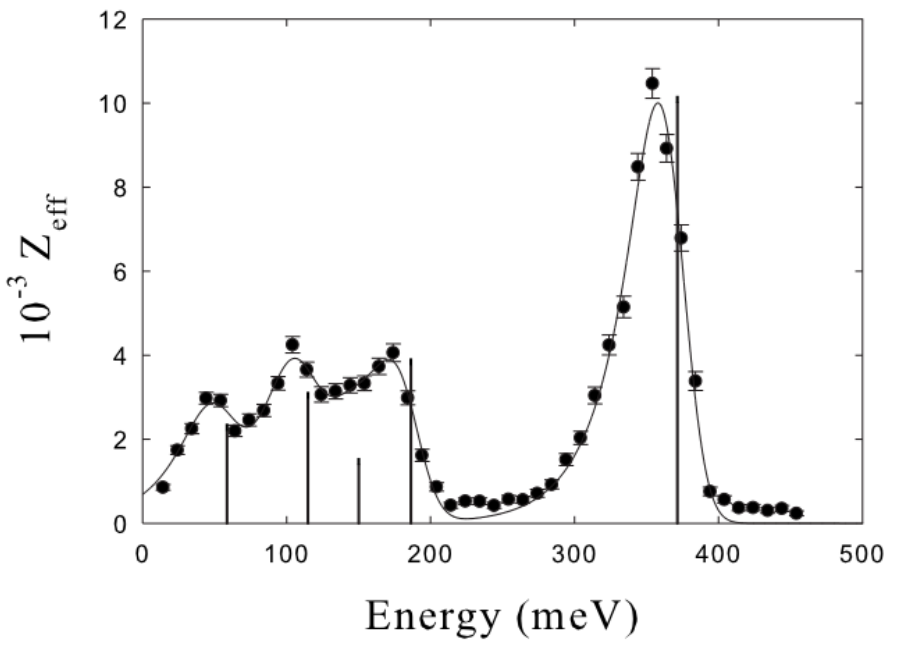

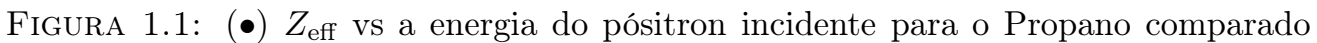
com as energias de transição vibracional do propano sem pósitron (linhas verticais)[42]

Esse comportamento foi associado a ressonâncias devidas a acoplamentos discreto-contínuo do pósitron na molécula que aconteciam simultâneamente com excitações vibracionais nela. Tal mecanismo é conhecido como uma ressonância vibracional de Feshbach (VFRs por suas siglas em inglês).[18]

O nome desses efeitos vem do formalismo de operadores de projeção de Feshbach que é utilizado, usualmente, na sua descrição[19-21]. A essência desse formalismo, ideado para lidar com problemas de física nuclear, é que as VFRs geralmente requerem que o alvo suporte estados localizados com a partícula incidente.[22, 23]. No caso do pósitron, a correlação da nuvem eletrônica e o pósitron permitirá um acoplamento da função de onda deslocalizada do pósitron em esses estados localizados. Equivalentemente, este efeito é 
entendido como uma ressonância, no regime da energia ou de um estado de tempo de vida finito no regime do tempo.

Uma vez que o pósitron encontra-se no estado localizado, a sua densidade de probabilidade terá uma sobreposição maior com a densidade de probabilidade dos elétrons de maneira que a aniquilação será aprimorada. No entanto, o tempo de vida médio desses estados é limitado pela ejeção do pósitron acompanhado por uma de-excitação vibracional e, na pratica, o pósitron precisara de se-acoplar mais de uma vez com as moléculas do gás. ${ }^{2}$

\subsection{Aspectos abordados no trabalho}

Este trabalho busca estudar, especificamente e desde um enfoque teórico-computacional, os mecanismos de acoplamento pósitron-molécula e as transições vibracionais na molécula que acontecem, simultaneamente, neste tipo de espalhamento, focando a atenção em efeitos não bem estudados anteriormente como os induzidos pela correlação pósitronelétron nos níveis vibracionais da molécula. Tudo isso, baseado nos dados experimentais disponíveis. Se apresentarão, como exemplo da inclusão desses efeitos, previsões no

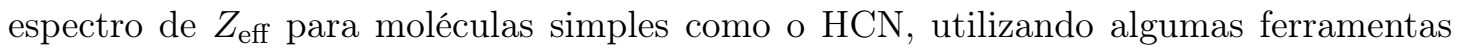
computacionais já desenvolvidas antes deste trabalho.

Mais explicitamente, será estudada a dinâmica de um sistema composto por três tipos de partículas: o pósitron, que no problema vai ser descrito por um estado resultante do acoplamento de estados deslocalizados e um estado localizado; os elétrons que em todo processo serão s discretos e, já que as transições entre esses estados requerem energia da ordem de alguns eV (maior a nosso regime), vamos supor que respondem de maneira adiabática. Finalmente, os núcleos que vão se movimentar num potencial induzido pelos elétrons e o pósitron. O movimento nuclear definirá os níveis de energia vibracionais cujas transições serão os fenômenos mais importantes estudados no trabalho.

Na primeira parte do texto, se apresentará a fundamentação teórica necessária para descrever os mecanismos de acoplamento contínuo-discreto do pósitron na ressonância e para descrever as vibracões da molécula. Serão introduzidas as notações que vão ser utilizadas no trabalho. Para o leitor, é recomendável focar sua atenção na introdução do formalismo de operadores de projeção de Feshbach e como caso específico, a aproximação de Gribakin-Lee que atualmente é uma das mais utilizadas para descrever a dinâmica do pósitron.

\footnotetext{
${ }^{2} \mathrm{O}$ tempo de vida típico de uma aniquilação simples elétron-pósitron é $\sim 10^{-10} \mathrm{~s}$ enquanto os tempos típicos de ressonância são da ordem de $\sim 10^{-12} \mathrm{~s}$
} 
Como trabalho original, foi proposto um modelo analítico para o cálculo das larguras de acoplamento do pósitron em transições Raman-Ativas mostrando que sua estimativa pode ser feita a partir de parâmetros da molécula isolada e tem uma ordem de grandeza comparável às larguras para acoplamentos em modos IR-ativos.

Também foi abordado o estudo teórico-computacional da correlação elétron-pósitron. A inclusão desse efeito, permite a excitação de vários níveis vibracionais do composto pósitron-molécula. São apresentados exemplos do mecanismo para moléculas diatômicas incluindo cálculos computacionais de estrutura eletrônica nas moléculas $\mathrm{LiH}$ e $\mathrm{NaH}$. Observaremos também as limitações do acoplamento do pósitron para moléculas com afinidade positrônica relativamente grande.

Finalmente, foi feita uma estimativa das propriedades de estrutura eletrônica e vibracional para a moléculas de mais de um modo normal de vibração. Especificamente trataremos á molécula de HCN apresentando um método para estimar, na aproximação harmônica, os efeitos de correlação elétron-pósitron na superfície de energia potencial.

Os temas desenvolvidos no trabalho contribuem a uma melhor compreensão teórica do fenômeno incluindo efeitos relevantes que nas aproximações atuais, principalmente fenomenológicas, não consideram. Esses efeitos (correlação pósitron-elétron e redistribuição vibracional de energia), embora vão ser estimados, a título de ilustração, para moléculas pequenas, nas quais os efeitos são modestos, esperamos que sejam importantes para moléculas maiores, que não podemos tratar computacionalmente no presente.

Por outro lado, a inclusão de ferramentas computacionais para o cálculo de estrutura eletrônica dos compostos pósitron-molécula é um grande aporte à explicação do problema. Essa técnica não tinha sido empregada no grupo de pesquisa e atualmente é um ramo ativo na física computacional. Este trabalho, representa, por tanto, o primeiro passo para um conjunto de projetos de pesquisa que seguramente vai vir no futuro.

\subsection{Organização do texto}

A introdução do formalismo teórico necessário para uma compressão dos aspectos desenvolvidos no trabalho, será abordada nos capítulos 2 até o 4 . No capítulo 5, será exposta a estimativa de larguras de acoplamento pósitron-molécula em modos vibracionais Raman-ativos. O mecanismo de acoplamento vibracional induzido pela correlação pósitron elétron e uma aplicação nas moléculas de $\mathrm{LiH}$ e NaH, será abordado no capítulo 6 e, finalmente, a generalização a sistemas com mais de um modo vibracional, tomando a molécula de HCN como exemplo, será apresentado no capítulo 7. 


\section{Capítulo 2}

\section{Introdução ao espalhamento ressonante}

O processo de espalhamento ressonante, embora seja uma fenômeno físico importante, usualmente não é introduzido nos cursos básicos de mecânica quântica de maneira que, neste capítulo, será feita uma introdução, principalmente qualitativa mas com certo formalismo teórico, do espalhamento ressonante.

\subsection{Descrição experimental do problema}

Em um processo de espalhamento, como o que é mostrado esquematicamente na figura 2.1, um feixe de partículas, colimado e quase-monoenergético, é dirigido em direção ao alvo que usualmente consiste em uma amostra de um grande número de centros espalhadores. Nosso problema, em particular, consiste em estudar o processo de espalhamento de pósitrons em gases moleculares, ou seja, sistemas de moléculas fracamente interagentes onde podem ser desprezados as colisões múltiplas.

Nos experimentos de aniquilação de pósitron, sobre um gás de de moléculas com uma densidade dada $(n)$, é medida a taxa de fótons de alta energia emitida como resultado de eventos da aniquilação relacionada com a taxa de incidência de pósitrons [18]. Essa taxa de eventos é normalizada com a taxa para um gás de elétrons livres à mesma densidade e é obtido o parâmetro $Z_{\text {eff }}$ [16] entendido, qualitativamente, como a quantidade de elétrons por molécula necessários para reproduzir a taxa de aniquilação desse experimento com respeito a experimentos de aniquilação simples pósitron-elétron.

Historicamente, as medidas de $Z_{\text {eff }}$ têm sido abordadas por dois métodos. O primeiro consistia en um gás de pósitrons, obtido por uma fonte radiativa de ${ }^{22} \mathrm{Na}$ os quais eram 


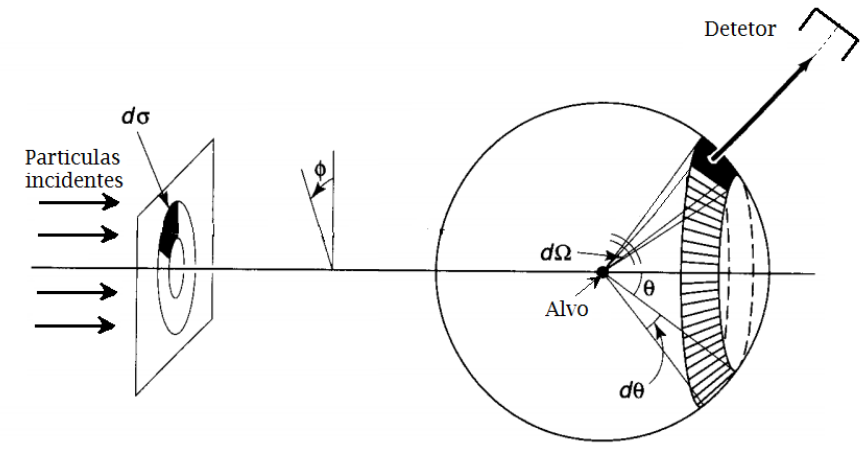

Figura 2.1: Representação esquemática do processo de espalhamento

armazenados en uma câmara e depois resfriados por um gás de $\mathrm{N}_{2}$ (gás onde o pósitron têm, tipicamente, uma vida média de $30 \mathrm{~s}$ a uma pressão de $10^{-7}$ Torr) até obter uma temperatura de $300 \mathrm{~K}$ para depois ser juntada com a amostra de gás que se deseja estudar. A taxa de eventos de aniquilação no tempo, era medida para obter o $Z_{\text {eff }}[24,25]$ o qual, rigorosamente correspondia a uma distribuiçõ térmica de energias.

O desenvolvimento de feixes aproximadamente monoenergeticos de pósitrons em 1997, permitiram a medida do espectro de $Z_{\text {eff }}$ como função da energia do pósitron incidente[17], permitindo descobrir que $Z_{\text {eff }}$ apresenta picos ressonantes para pósitrons com energias específicas. Nas aproximações teóricas no intervalo de enervia de $0 \mathrm{eV}$ até $0.5 \mathrm{eV}$, que têm sido principalmente fenomenológicas, a aniquilação do pósitron é intermediada pela formação de um composto pósitron-molécula, excitando os níveis vibracionais dela. Para obter uma solução da função de onda $\Psi\left(\mathbf{r}_{1}, \cdots, \mathbf{r}_{2}, \mathbf{r}_{p}\right)$ do sistema, é apresentada a seguinte seção com alguns aspectos úteis e que vão ser usados ao longo do texto.

\subsection{Descrição da teoria de espalhamento ressonante}

Esta seção tem como objetivo, familiarizar ao leitor com fenômeno de ressonância de Feshbach de uma maneira não muito formal mas qualitativamente inteligível. Em lugar de lidar aqui com a situação mais geral que é incluída no formalismo de Feshbach, vamos limitar-nos a um modelo simples de maneira que o formalismo não seja um obstáculo. Não vamos incluir todos os detalhes do fenômeno mas explicaremos alguns resultados.

Em geral, a função de onda do pósitron, no problema de espalhamento, corresponde a uma combinação de estados discretos e de estados do contínuo. Na teoria de perturbações, usualmente as soluções a primeira ordem são obtidas a partir de estados 
conhecidos (aproximações de ordem zero) que posteriormente são acoplados por um potencial. Tomaremos sem entrar em detalhes, como estados a ordem zero, a função de onda deslocalizada $|E\rangle$ com energia $E$ e a função de onda localizada $\left|\phi_{d}\right\rangle$ com energia $E_{d}$. Esses estados são importantes nos límites assintóticos (muito longe ou muito perto da molécula) de maneira que é colocado um potencial $V$ para acoplar os dois estados modelado de tal maneira que consiga descrever razoavelmente a interação do pósitron na região intermediaria.

O resultado essencial é que o estado localizado $\left|\phi_{d}\right\rangle$ somente vai ser acoplado a estados do contínuo com energia próxima à dele dentro de um intervalo de largura $\Gamma$ que, pode ser relacionado à taxa de transição do estado discreto até o contínuo calculado usando a regra de ouro de Fermi. Explicaremos como essa dissolução do estado discreto no conjunto de estados dentro desse intervalo com largura $\Gamma$ implica o decaimento exponencial do estado discreto. Finalmente apresentaremos o que acontece se além do potencial $(V)$ de acoplamento contínuo-discreto do pósitron são ligados, simultaneamente acoplamentos entre os níveis discretos do alvo mediante um potencial $U$. Finalmente vai ser definido o operador de transição $T_{i j}$. Antes de começar, vai ser esclarecido que, no trabalho, serão usadas as unidades atômicas.

\subsubsection{Operador de transição $T$}

Em um problema perturbativo, usualmente a partir de um Hamiltoniano $H_{0}$ cujo espectro é conhecido, é procurada a solução para o mesmo problema mas com um potencial $V$ a partir dos auto estados $\left|\phi_{0}\right\rangle$ de $H_{0}$

$$
H=H_{0}+V
$$

Definindo o autoestado $(|\psi\rangle)$ de $H$ de maneira que satisfaça a equação de Schrödinger $\left(H_{0}+V\right)|\psi\rangle=E|\psi\rangle$, sua solução geral satisfaz:

$$
|\psi\rangle=\frac{1}{E-H_{0}} V|\psi\rangle+\left|\phi_{0}\right\rangle
$$

onde se tem definido o operador $\left(E-H_{0}\right)^{-1}$ como o operador inverso do operador $E-H_{0}$ e a função $\left|\phi_{0}\right\rangle$ é introduzida uma vez que é o limite de $|\psi\rangle$ quando $V \rightarrow 0$.

Usualmente, para satisfazer as condições de contorno, introduz-se uma fraca parte imaginaria $\varepsilon$ de forma que a equação fica:

$$
\left|\psi^{( \pm)}\right\rangle=\frac{1}{E-H_{0} \pm i \varepsilon} V\left|\psi^{ \pm}\right\rangle+\left|\phi_{0}\right\rangle
$$


A solução para $\varepsilon$ positivo (negativo) corresponde á onda incidente mais a uma onda esférica espalhada que sai (que entra). Em uma grande parte dos problemas físicos, a condição de contorno usual é satisfeita pela solução positiva. Com ajuda dessa equação, é definido o operador de transição $T$ de forma que ele satisfaz:

$$
V\left|\psi^{( \pm)}\right\rangle=T_{( \pm)}\left|\phi_{0}\right\rangle
$$

Portanto, multiplicando a equação (2.3) por $\mathrm{V}$, pode ser concluído que o operador $T$ satisfaz:

$$
T_{( \pm)}=V+V \frac{1}{E-H_{0} \pm i \varepsilon} T_{( \pm)}
$$

que vai ter diferentes soluções dependendo do tipo de potencial que é utilizado. Na seguinte seção vai ser observado o quê acontece quando o Hamiltoniano $H$ admite acoplamentos entre níveis discretos e níveis contínuos intermediado pelo potencial $(V)$.

\subsubsection{Acoplamento entre um estado discreto e estados do contínuo}

As ressonâncias, em um processo de espalhamento, acontecem devido ao acoplamento de níveis discretos de energia com níveis contínuos. As primeiras aproximações teóricas ao mecanismo de acoplamento foram estudadas por Fano em 1935[26]. A aproximação apresentada aquí, é baseada no texto de Cohen-Tannoudji[27]

Considerando o Hamiltoniano

$$
H=H_{0}+V
$$

onde os autoestados de $H_{0}$ (sem emtrar em detalhes) incluem: um estado localizado $\left|\phi_{d}\right\rangle$ e estados deslocalizados com espectro de energia contínuo $|E\rangle$ sendo $E$ o autovalor associado à energia. Definindo eles como estados de ordem zero, válidos na região assintótica, a solução a primeira ordem vai ser uma superposição desses estados, da maneira

$$
|\psi\rangle=\langle E \mid \psi\rangle|E\rangle+\left\langle\phi_{d} \mid \psi\right\rangle\left|\phi_{d}\right\rangle
$$

Considerando o acoplamento entre eles mediante um potencial $V$ onde o elemento de matriz $\left\langle E|V| \phi_{d}\right\rangle=v(E)$ é uma função arbitrária de $E$. Uma vez que $\left|\phi_{d}\right\rangle$ e $|E\rangle$ correspondem a auto-estados do Hamiltoniano de ordem zero $H_{0}$, podemos impor que eles 
satisfazem:

$$
\begin{aligned}
\left\langle\phi_{d}\left|H_{0}\right| \phi_{d}\right\rangle & =E_{\phi} \\
\left\langle E\left|H_{0}\right| E^{\prime}\right\rangle & =E \delta\left(E-E^{\prime}\right) \\
\left\langle\phi_{d}|V| E\right\rangle & =v(E) \\
\left\langle E|V| E^{\prime}\right\rangle & =\left\langle\phi_{d}|V| \phi_{d}\right\rangle=0
\end{aligned}
$$

onde $\delta(x)$ é a distribuição delta de Dirac.

Seja $\left|\psi\left(E^{\prime}\right)\right\rangle$ o autoestado de $H$ com autovalor $E^{\prime}$

$$
H\left|\psi\left(E^{\prime}\right)\right\rangle=E^{\prime}\left|\psi\left(E^{\prime}\right)\right\rangle
$$

queremos encontrar uma expansão de $\left|\psi\left(E^{\prime}\right)\right\rangle$ nos estados assintóticos $\left|\phi_{d}\right\rangle$ e $|E\rangle$. A solução de (2.9) satisfaz a equação de Lippmann-Schwinger:

$$
\left|\psi\left(E^{\prime}\right)\right\rangle=\left|E^{\prime}\right\rangle+\frac{1}{E^{\prime}-H_{0}} V\left|\psi\left(E^{\prime}\right)\right\rangle
$$

Onde tem sido adicionado o estado $\left|E^{\prime}\right\rangle$ que corresponde á solucão quando $V \rightarrow 0$. Primeiro, projetando (2.10) em $\left\langle\phi_{d}\right|$ e depois em $\langle E|$ obtemos as equações

$$
\begin{aligned}
\left\langle\psi_{d} \mid \psi\left(E^{\prime}\right)\right\rangle & =\frac{1}{E^{\prime}-E_{\phi}} \int d E^{\prime \prime}\left\langle\phi_{d}|V| E^{\prime \prime}\right\rangle\left\langle E^{\prime \prime} \mid \psi\left(E^{\prime}\right)\right\rangle \\
\left\langle E \mid \psi\left(E^{\prime}\right)\right\rangle & =\delta\left(E-E^{\prime}\right)+\frac{1}{E^{\prime}-E}\left\langle E|V| \phi_{d}\right\rangle\left\langle\phi_{d} \mid \psi\left(E^{\prime}\right)\right\rangle
\end{aligned}
$$

Uma vez que $1 /\left(E^{\prime}-E\right)$ pode ter varios valores num intervalo continuo, utilizando a teoria de distribuições, [28] a solução geral de (2.12) pode ser escrita da maneira:

$$
\left\langle E \mid \psi\left(E^{\prime}\right)\right\rangle=\left[\wp \frac{1}{E^{\prime}-E}-\pi i \delta\left(E-E^{\prime}\right)\right] v(E)\left\langle\phi_{d} \mid \psi\left(E^{\prime}\right)\right\rangle+\delta\left(E-E^{\prime}\right)
$$

sendo $\wp$ a parte principal de Cauchy. Assim, substituindo (2.13) em (2.11), é obtido:

$$
\left\langle\phi_{d} \mid \psi\left(E^{\prime}\right)\right\rangle=\frac{v\left(E^{\prime}\right)}{\left(E^{\prime}-E_{\phi}-\Delta\left(E^{\prime}\right)\right)+i \frac{\Gamma\left(E^{\prime}\right)}{2}},
$$

com

$$
\begin{aligned}
\Gamma\left(E^{\prime}\right) & =2 \pi\left|v\left(E^{\prime}\right)\right|^{2} \\
\Delta\left(E^{\prime}\right) & =\wp \int d E \frac{|v(E)|^{2}}{E^{\prime}-E}=\frac{1}{2 \pi} \int d E \frac{\Gamma(E)}{E^{\prime}-E}
\end{aligned}
$$


Supondo que definimos o estado $|\Psi\rangle$, que em $t=0$ encontra-se, inteiramente, no estado deslocalizado $\left|\phi_{d}\right\rangle$. Dessa maneira, usando a relação (2.14), expandindo $\left|\phi_{d}\right\rangle$ na base de auto-estados do Hamiltoniano $|\psi\rangle$, obtemos:

$$
\left.|\Psi\rangle\right|_{t=0^{+}}=\left|\phi_{d}\right\rangle=\int d E^{\prime} \frac{v^{*}\left(E^{\prime}\right)}{\left(E^{\prime}-E_{\phi}-\Delta\left(E^{\prime}\right)\right)-i \frac{\Gamma\left(E^{\prime}\right)}{2}}\left|\psi\left(E^{\prime}\right)\right\rangle .
$$

Uma vez que, $E^{\prime}$ é auto estado do Hamiltoniano, a evolução temporal de $|\psi\rangle$ é dada por:

$$
|\Psi(t)\rangle=\int d E^{\prime} \frac{v^{*}\left(E^{\prime}\right) e^{-i E^{\prime} t}}{\left(E^{\prime}-E_{\phi}-\Delta\left(E^{\prime}\right)\right)-i \frac{\Gamma\left(E^{\prime}\right)}{2}}\left|\psi\left(E^{\prime}\right)\right\rangle .
$$

e a amplitude de probabilidade de encontrar o sistema, de novo, no estado $\left|\phi_{d}\right\rangle$ é igual a:

$$
\left\langle\phi_{d} \mid \Psi(t)\right\rangle=\int \frac{\Gamma\left(E^{\prime}\right)}{2 \pi} \frac{e^{-i E^{\prime} t / \hbar}}{\left(\frac{\Gamma\left(E^{\prime}\right)}{2}\right)^{2}+\left(E^{\prime}-E_{\phi}-\Delta\left(E^{\prime}\right)\right)^{2}} d E^{\prime} .
$$

onde temos usado a relação (2.15). Considerando $v(E)$ constante em um intervalo de energia $E \in\left(E_{\phi}+\Delta\left(E_{\phi}\right)-\Gamma\left(E_{\phi}\right), E_{\phi}+\Delta\left(E_{\phi}\right)+\Gamma\left(E_{\phi}\right)\right)$, podemos fazer as aproximações:

$$
\begin{aligned}
\Delta\left(E^{\prime}\right) & \approx \Delta\left(E_{\phi}\right) \equiv \Delta \\
\Gamma\left(E^{\prime}\right) & \approx \Gamma\left(E_{\phi}+\Delta\right) \equiv \Gamma_{r}
\end{aligned}
$$

a integral (2.19) pode ser calculada usando o método de resíduos e é reduzida até:

$$
\langle\phi \mid \Psi(t)\rangle=e^{-\Gamma_{r}|t| / 2}
$$

Dessa maneira, a probabilidade de encontrar o sistema no instante $t$ no estado discreto $\left|\phi_{d}\right\rangle$ é, portanto

$$
|\langle\phi \mid \Psi(t)\rangle|^{2}=e^{-\Gamma_{r}|t|}
$$

Isto é, a probabilidade de encontrar, no estado localizado, um sistema que inicialmente encontrava-se nele, decai exponencialmente no tempo e com um tempo de vida dado por $\tau=1 / \Gamma_{r}$. Portanto, podemos concluir que todo estado discreto acoplado a um estado contínuo vai apresentar uma instabilidade e por isso, geralmente esses estados são chamados meta-estáveis.

Se é considerado o intervalo de energia $[E, E+d E]$ com $d E$ pequeno com respeito a $\Gamma_{r}$, a probabilidade $d P_{r}$ de encontrar a partícula no estado discreto $\left|\phi_{d}\right\rangle$ nesse intervalo de energia é igual a:

$$
d P_{r}=\sum_{E<E_{\psi}<E+d E}|\langle\phi \mid \psi\rangle|^{2} \approx d E|\langle\phi \mid \psi\rangle|^{2}
$$


que, usando a relação (2.14), fica:

$$
\frac{d P_{r}}{d E}=\frac{\Gamma_{r} / 2 \pi}{\left(\frac{\Gamma_{r}}{2}\right)^{2}+\left(E-E_{\phi}-\Delta\right)^{2}}
$$

que é a equação de uma curva conhecida como perfil de Breit-Wigner[29] com largura $\Gamma_{r}$ e centrada em $E=E_{r}=E_{\phi}+\Delta$ como a mostrada na figura (2.2).

A ideia principal do que acontece em um espalhamento ressonante é que, durante o acoplamento, o nível discreto $\left|\phi_{d}\right\rangle$ distribui-se sobre os estados deslocalizados $|E\rangle$ com energia centrada em $E_{r}$ e dentro de um intervalo de largura $\Gamma_{r}$. Assim, para ressonâncias que decaem rapidamente, seu $\Gamma_{r}$ associado vai ser muito grande entretanto que para ressonâncias com tempo de vida longo, esse intervalo é bem pequeno pois ocorrem em um intervalo de energia muito bem definida.

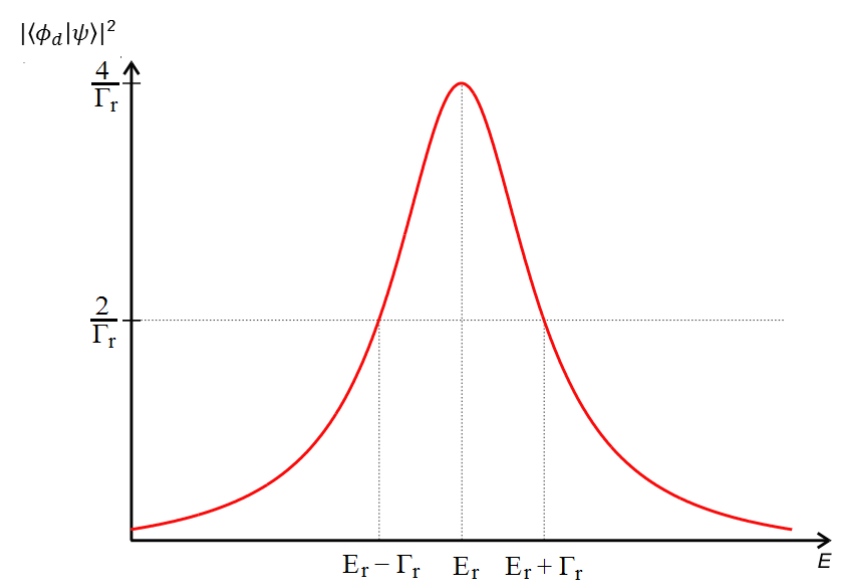

Figura 2.2: Gráfico de uma parâmetro ressonância, como a descrita pela equação (2.25), centrada em $E_{r}$ e com largura $\Gamma_{r}$

Para calcular a sobreposição de densidades como na equação (1.1), como vai ser demostrado na seção 4.2.1, a principal contribuição vai ser proporcional ao produto de componentes discretas da função de onda do pósitron $\left(\left|\phi_{d}\right\rangle\right)$ descritas pela equação (2.2) portanto, o parâmetro de aniquilação, vai ter a forma:

$$
Z_{\text {eff }} \propto\left\langle\phi \mid \phi_{d}\right\rangle \propto \sum_{r} \frac{\Gamma_{r}}{\left(E-E_{r}\right)^{2}+\Gamma_{r}^{2} / 4}
$$

A equação (2.25) apresenta um espectro de $Z_{\text {eff }}$ com picos nas energias de ressonância $E=E_{r}$ como o apresentado na figura (2.2) cuja forma vai depender do parâmetro $\Gamma_{r}$ que como na equação (2.22) descreve a taxa de decaimento do estado. 


\subsection{Descrição da excitação de níveis discretos do alvo}

Nesta seção apresentaremos algumas considerações qualitativas para ilustrar a física por trás das ressonâncias que produzem transições entre níveis discretos do alvo. Os termos que incluiremos aqui, são obtidos naturalmente ao considerar a teoria de Feshbach, aqui precisaremos fazer algumas suposições.

Assumindo que o alvo possui algum outro estado localizado $|\chi\rangle$ com energia $E_{\chi}$, ortogonal ao estado $\left|\phi_{d}\right\rangle$ acoplado com o estado assintoticamente livre $\{|E\rangle\}$ supondo a existência de um potencial $U$ interno do alvo que somente faz o acoplamento entre os estados discretos deste; $|\chi\rangle$ e $\left|\phi_{d}\right\rangle$ :

$$
\begin{aligned}
& \langle\phi|U| \chi\rangle=u \\
& \langle E|U| \chi\rangle=0
\end{aligned}
$$

com os demais elementos de matriz de $U$ nulos.

Uma vez que, na seção anterior, temos resolvido os estados $|\psi\rangle$ como combinação do estado discreto $\left|\phi_{d}\right\rangle$ e o continuo $|E\rangle$, ao calcular $\langle\psi|U| \chi\rangle$ uma vez $U$ faz o acoplamento de $|\chi\rangle$ somente com $\left|\phi_{d}\right\rangle$

$$
\langle\psi|U| \chi\rangle=\left\langle\psi \mid \phi_{d}\right\rangle\langle\phi|U| \chi\rangle=\left\langle\psi \mid \phi_{d}\right\rangle u
$$

Se o termo $u$ não é muito grande, a taxa de transição das duas transições simultâneas pode ser calculada utilizando a regra de ouro de Fermi:

$$
\Gamma_{\chi}=\frac{2 \pi}{\hbar}|\langle\psi|U| \chi\rangle|^{2} \frac{1}{\delta}
$$

onde $1 / \delta$ é a densidade de estados do estado discreto-contínuo $|\psi\rangle$. A partir das equações $(2.14)$ e (2.27), vemos que a taxa, no limite $\delta \rightarrow 0$ pode ser escrita da maneira:

$$
\Gamma_{\chi}=u^{2} \frac{\Gamma_{r}}{\left(\frac{\Gamma_{r}}{2}\right)^{2}+\left(E_{\chi}-E_{r}\right)^{2}}
$$

de maneira que, na ressonância, $\Gamma_{\chi}=4 u^{2} / \Gamma_{r}$

Em conclusão, se acontecer uma ressonância que acople o nível continuo $\left|E_{r}\right\rangle$ e o nível discreto $\left|\phi_{d}\right\rangle$ estando este último também acoplado por um potencial interno $U$ a outo nível discreto $|\chi\rangle,\left|E_{r}\right\rangle$ estará acoplado com $|\chi\rangle$ embora $\left\langle E_{r}|V| \chi\right\rangle=0$. Tais acoplamentos serão, também ressonantes. Este resultado justifica as transições vibracionais feitas no alvo induzidas, simultaneamente, pelo acoplamento pósitron-molécula. 


\subsection{Operador de transição em um espalhamento ressonante incluindo transições dos níveis do alvo}

Considerando dois níveis $\left|\chi_{i}\right\rangle$ e $\left|\chi_{j}\right\rangle$ com a mesma energia $E_{\chi}$ acoplados, pelo potencial $U$, com o estado $\left|\phi_{d}\right\rangle\left(\left\langle\phi|U| \chi_{i}\right\rangle=u_{i}\right.$ e $\left.\left\langle\phi|U| \chi_{j}\right\rangle=u_{j}\right)$ e que não estão acoplados entre eles $\left(\left\langle\chi_{i}|U| \chi_{j}\right\rangle=0\right)$ nem com os níveis do contínuo $\{|E\rangle\}\left(\left\langle E|U| \chi_{j}\right\rangle=0\right)$. A matriz de transição entre os estados $\left|\chi_{i}\right\rangle$ até $\left|\chi_{j}\right\rangle$, definida na equação (2.5) e obtida a partir da solução de $H=H_{0}+V$ incluindo o potencial $U$ que faz transições internas do alvo como potencial perturbativo, é dada por:

$$
T_{i j}=\lim _{\varepsilon \rightarrow 0_{+}}\left\langle\chi_{i}|U| \phi_{d}\right\rangle\left\langle\phi\left|\frac{1}{E_{\chi}-H+i \varepsilon}\right| \phi_{d}\right\rangle\left\langle\phi|U| \chi_{j}\right\rangle
$$

onde $H=H_{0}+V$ com $V$ o potencial de acoplamento discreto-contínuo. Introduzindo uma relação de completeza dos auto-estados $|\psi(E)\rangle$ de $H$ e utilizando as equações (2.14) e $(2.27)$ :

$$
T_{i j}=u_{j}^{*} u_{i} \lim _{\varepsilon \rightarrow 0_{+}} \int_{-\infty}^{\infty} d E \frac{\hbar \Gamma_{r} / 2 \pi}{\left(E_{\chi}-E-E_{r}+i \varepsilon\right)\left(\left(E-E_{r}\right)^{2}+\left(\frac{\hbar \Gamma_{r}}{2}\right)^{2}\right)}
$$

que utilizando o método de resíduos, fica simplesmente:

$$
T_{i j}=u_{j}^{*} u_{i} \frac{1}{\left(E_{\chi}-E_{r}\right)+i \hbar\left(\Gamma_{r} / 2\right)}
$$

observamos que tudo acontece como se, na expressão de primeira ordem, a energia do nível discreto $\left|\phi_{d}\right\rangle$, fosse substituída por uma energia complexa $E_{\phi}=E_{\phi}+\Delta-i \Gamma / 2$ enquanto que a matriz de transição tem um comportamento ressonânte quando $E_{\chi}$ percorre um intervalo de energia centrado em $E_{r}$ e com largura $\Gamma_{r}$. 


\section{Capítulo 3}

\section{Graus de liberdade vibracionais da molécula}

Uma vez tem sido conceitualmente entendido o acoplamento contínuo-discreto que acontece num espalhamento induzindo uma ressonância, vai ser feita uma introdução breve ao formalismo utilizado no texto para a descrição das vibrações de uma molécula assim como as transições vibracionais. Uma aproximação mais profunda pode ser encontrada no texto de Bright, Decius e Cross. [30]

\subsection{A superfície de energia potencial}

Nosso problema, tem a desvantagem de lidar simultaneamente com pósitrons, elétrons e núcleos atómicos. No entanto, usualmente o problema eletrônico não é resolvido de maneira simultânea ao nuclear. Este desacoplamento é conhecido como aproximação Born-Oppenheimer[31]. Suportado na ideia que, uma vez que a massa dos núcleos é muito maior do que a massa dos elétrons, no tempo típico de movimento eletrônico, os núcleos parecem estar fixos e no tempo típico de movimento nuclear, as interações dos elétrons respondem instantaneamente e podem ser tratadas como um potencial efetivo com dependência paramétrica com as coordenadas nucleares. ${ }^{1}$

Começamos definindo o Hamiltoniano total:

$$
H=T_{p}+T_{N}+T_{\text {ele }}+V_{\text {ele-ele }}+V_{\text {ele }-N}+V_{p-\text { mol }}=T_{N}+T_{p}+H_{\text {ele }}
$$

\footnotetext{
${ }^{1}$ Em grande parte dos casos, os tempos típicos de movimento dos elétrons $\left(\sim 10^{-17} s\right)$ (formação do transiente e excitações eletrônicas) são muito menores do que os tempos de movimento dos núcleos (rotação $\sim 10^{-12} s$ e vibração $\sim 10^{-14} s$ ).
} 
com $T_{p}+T_{N}+T_{\text {ele }}$ as energias cinéticas do pósitron $(p)$, os núcleos $(N)$ e os elétrons (ele) e os potenciais de interação elétron-elétron $\left(V_{\text {ele-ele }}\right)$, elétrons-núcleos $\left(V_{\text {ele- }} N\right)$ e pósitron-molécula $\left(V_{p-\text { mol }}\right)$ os quais definem o hamiltoniano eletrônico $\left(H_{\text {ele }}\right)$.

Explicitamente, $H_{\text {ele }}$ pode ser escrito da maneira:

$$
H_{\text {ele }}=\sum_{i=1}^{n} \frac{\mathbf{p}_{i}^{2}}{2 m_{i}}+\sum_{i>j} \frac{e^{2}}{\left|\mathbf{r}_{i}-\mathbf{r}_{j}\right|}+\sum_{I>J} \frac{Z_{I} Z_{J} e^{2}}{\left|\mathbf{R}_{I}-\mathbf{R}_{J}\right|}-\sum_{i} \sum_{I} \frac{Z_{I} e^{2}}{\left|\mathbf{r}_{i}-\mathbf{R}_{J}\right|}
$$

onde $\{\mathbf{r}\}$ é o conjunto de coordenadas eletrônicas e do pósitron $\{\mathbf{R}\}$ o conjunto das coordenadas dos núcleos atômicos, cada um, com número atómico $Z_{I}$.

O autoestado eletrônico (e positrônico eventualmente) $\left(\Phi_{\text {ele }}(\mathbf{r} \mid \mathbf{R})\right)$ de $H_{\text {ele }}$ dependente parametricamente da configuração $\{\mathbf{R}\}$ dos núcleos, satisfaz a equação de Schrödinger não dependente do tempo:

$$
H_{\text {ele }} \Phi_{0}(\mathbf{r} \mid \mathbf{R})=E_{\text {ele }}(\mathbf{R}) \Phi_{0}(\mathbf{r} \mid \mathbf{R})
$$

Uma vez que $H_{\text {ele }}$ vai depender somente das coordenadas eletrônicas, depois de ser resolvido para cada configuração $(\mathbf{R})$ possível, no espaço dos núcleos, o auto valor $E_{\text {ele }}(\mathbf{R})$ é tratado como um potencial efetivo dependente da configuração cujo valor para cada uma delas permite construir a Superfície de Energia Potencial (SEP) que vai ser utilizada nos cálculos vibracionais. A solução da equação (3.3) no espaço eletrônico pode ser atacada como diversos métodos computacionais que vão ser utilizados em nosso trabalho e que são introduzidos no apêndice A.

\subsection{Vibrações moleculares}

Uma vez resolvido o hamiltoniano eletrônico usando qualquer método de cálculo de estrutura eletrônica para diferentes configurações nucleares $\mathbf{R}$, a parte do hamiltoniano total que descreve a dinâmica nuclear pode ser escrita da maneira:

$$
\left\{\sum_{i} \frac{P_{i}^{2}}{2 M_{i}}+E_{\text {ele }}(\mathbf{R})\right\}|\Phi\rangle \equiv\left(T_{N}+V(\mathbf{R})\right)|\Phi\rangle
$$

Com $T_{N}$ o operador de energia cinética dos núcleos e $V(\mathbf{R})=E_{\text {ele }}(\mathbf{R})$ um potencial efetivo. Para encontrar autovalores aproximadoes de (3.4), usualmente $V(\mathbf{R})$ é expandida em serie de potencias de até segunda ordem, em relação com as coordenadas dos núcleos $\left\{\mathbf{R}_{i}\right\}$ medidas ao redor da geometria de equilíbrio $\left(\mathbf{R}_{0}\right)$, definida como aquela onde $V(\mathbf{R})$ é mínimo, de maneira que utilizando as coordenadas de deslocamentos $\left\{\delta R_{i}\right\}$ com 
respeito a $\mathbf{R}_{0}$ :

$$
V(\delta \mathbf{R})=V(0)+\sum_{i}\left(\frac{\partial V}{\partial R_{i}}\right)_{0} \delta R_{i}+\frac{1}{2} \sum_{i, j}\left(\frac{\partial^{2} V}{\partial R_{i} \partial R_{j}}\right)_{0} \delta R_{i} \delta R_{j}+\cdots
$$

onde o sub-índice $i$ leva a conta por cada núcleo e seus graus de liberdade. De aqui, fixando $V(0)=0$, como a referencia de energia potencial, e uma vez que a derivada da energia nesse ponto é nula, para pequenos deslocamentos a redor do equilíbrio, definimos:

$$
V(\delta \mathbf{R})=\frac{1}{2} \sum_{i, j} k_{i, j} \delta R_{i} \delta R_{j} \quad k_{i, j} \equiv\left(\frac{\partial^{2} V}{\partial R_{i} \partial R_{j}}\right)_{0}
$$

onde $k_{i j}$ é a constante generalizada de força. Definindo as coordenadas $\left(q_{i}\right)$ ponderadas pela massa $\left(m_{\alpha}\right)$ do deslocamento $i$ da partícula $\alpha$ da maneira:

$$
q_{i}=m_{\alpha}^{1 / 2} \delta R_{i}
$$

o hamiltoniano pode ser escrito da maneira:

$$
H=\frac{1}{2} \sum_{i} \dot{q}_{i}^{2}+\frac{1}{2} K_{i j} q_{i} q_{j}
$$

O problema é encontrar o conjunto de combinações lineares $Q_{i}$ das coordenadas $q_{i}$ de maneira que o Hamiltoniano pode ser escrito como um operador diagonal na maneira:

$$
H=\frac{1}{2} \sum_{i} \dot{Q}_{i}^{2}+\frac{1}{2} \lambda_{i} Q_{i}^{2}
$$

Nesse sistema de coordenadas, a equação de Schrödinger, é facilmente solúvel permitindo obter os níveis de energia:

$$
E=\sum_{i}\left(\nu_{i}+\frac{1}{2}\right) \hbar \omega_{i}
$$

onde $\lambda_{i}=\omega_{i}^{2}$ e a função de onda total é o produto de soluções independentes $|\chi\rangle=$ $\left|\nu_{1}\right\rangle\left|\nu_{2}\right\rangle \cdots$ cada uma, solução da equação do oscilador harmônico com número quântico $\nu_{i}$ que designa o nível $\nu$ do modo $i$-ésimo. Para aqueles modos que são rotações ou translações (em total 6), ${ }^{2} \lambda_{i}=0$ de maneira que é restringido o número de modos vibracionais a $3 N-6$ sendo $N$ a quantidade de núcleos.

\subsubsection{Transições vibracionais IR-ativas}

As linhas espectrais de absorção e emissão de luz para uma molécula surgem de transições eletrônicas, rotacionais e vibracionais. As transições rotacionais ocorrem no regime de

\footnotetext{
${ }^{2}$ Se a molécula é linear, são 5.
} 
micro-ondas, as transições eletrônicas ocorrem no regime de luz visível e ultravioleta, enquanto que as transições vibracionais ocorrem no regime infravermelho e visível. A faixa infravermelha, correspondente a transições, principalmente, vibracionais é a que vai ser mais importante para nós.

As transições vibracionais de maior importância são induzidas pela interação da componente elétrica do campo eletromagnético com a componente do momento de dipolo da molécula paralela ao campo. Quanticamente, como é apresentado no apêndice B a interação de um campo eletromagnético com um dipolo obedece a uma transição entre o estado inicial $|i\rangle$ e o estado final $|f\rangle$ da molécula cuja intensidade, nesse caso obedece á equação (B.33)

$$
A=\frac{\pi N_{A} \omega_{f i}}{3 \hbar c}|\langle i|\boldsymbol{\mu}| f\rangle|^{2}=\frac{\pi N_{A} \omega_{f i}}{3 \hbar c} \mu_{f i}^{2}
$$

onde $N_{A}$ é o número de Avogadro, $\omega_{f i}$ é a frequência do fóton associado à transição e $\boldsymbol{\mu}$ é o operador vetorial de momento de dipolo cuja definição das suas componentes é dada a partir as coordenadas $x_{\alpha}$ das cargas $e_{\alpha}$ das partículas $(\alpha)$ que compõem a molécula:

$$
\mu_{x}=\sum_{\alpha} e_{\alpha} x_{\alpha}
$$

No entanto, ao levar em consideração as vibrações nucleares, as cargas poderão, em geral, se rearranjar em razão do movimento nuclear. Portanto, usando as coordenadas normais $Q_{k}$ do deslocamento, as componentes $\mu_{i}$ do operador de momento dipolar, podem ser escritas como:

$$
\mu_{i}=\mu_{i}^{0}+\sum_{k}\left(\frac{\partial \mu_{i}}{\partial Q_{k}}\right)_{0} Q_{k}+\cdots
$$

onde, $k$ leva conta dos modos normais (em total $3 N-6$ com $N$ o número de átomos) e foi assumido que os termos de ordem superior são negligenciáveis. Partindo dela, é concluído que para uma transição vibracional de primeira ordem, o elemento de matriz $\boldsymbol{\mu}_{\nu_{k}^{\prime} \nu_{k}}$ para a excitação do modo $k$-ésimo, satisfaz as regras de seleção:

$$
\begin{aligned}
\boldsymbol{\mu}_{\nu_{k}^{\prime} \nu_{k}} & =\left\langle 0 \cdots \nu_{k}^{\prime} \cdots 0|\boldsymbol{\mu}| 0 \cdots \nu_{k} \cdots 0\right\rangle=\left(\frac{\partial \boldsymbol{\mu}}{\partial Q_{k}}\right)_{0}\left\langle\nu_{k}^{\prime}\left|Q_{k}\right| \nu_{k}\right\rangle \\
& =\left(\frac{\partial \boldsymbol{\mu}}{\partial Q_{k}}\right)_{0}\left(\frac{1}{2 \omega_{k}}\right)^{1 / 2}\left(\delta_{\nu^{\prime}, \nu_{k}+1}\left(\nu_{k}+1\right)^{1 / 2}+\delta_{\nu_{i}^{\prime}, \nu_{i}-1}\left(\nu_{k}\right)^{1 / 2}\right)
\end{aligned}
$$

Ou simplesmente: $\nu_{k}^{\prime}=\nu_{k} \pm 1$. Usualmente, o estado fundamental é o mais ocupado de maneira que é válido lidar simplesmente, com transições de absorção de $\nu_{k}=0$ até $\nu_{k}^{\prime}=1$ de maneira que o elemento de matriz pode ser escrito simplesmente $\mu_{k}$ e a energia associada a esta transição simplesmente $\hbar \omega_{k}$. Assim, o modo $Q_{k}$ (que involve o movimento, em geral, de muitos átomos) cujo deslocamento induz uma mudança no momento de dipolo, ou seja, para o qual $\left(\partial \boldsymbol{\mu} / \partial Q_{k}\right)_{0} \neq 0$; usualmente é chamado ativo 
no infravermelho pois sua excitação pode contribuir a o espectro de absorção ou emissão vibracional nessa região do espectro.

Em seções seguintes, vai ser útil o cálculo da quantidade $\omega_{\nu} \mu_{\nu}^{2}$ que pode ser obtida a partir das mediações experimentais de (3.11). Em nosso caso, da partir dos cálculos computacionais de estrutura eletrônica, obteremos $A_{\nu}$ em unidades de $\left(\operatorname{Deybe}^{2} /\left(\mathrm{AMU}^{*} \AA^{2}\right)\right.$. De maneira que $\omega_{\nu} \mu_{\nu}^{2}$ pode ser obtido, partindo de $A_{\nu}$, utilizando a equação:

$$
\omega_{\nu} \mu_{\nu}^{2}(\mathrm{meV})=\left(\frac{1}{84106.6}\right)\left(\frac{A_{\nu}}{g_{\nu}}\right)
$$

Onde $g_{\nu}$ é a degenerescência do modo $\nu$

\subsubsection{Transições vibracionais Raman-ativas}

A magnitude de $\boldsymbol{\mu}$ pode mudar pela magnitude do campo elétrico que perturba à molécula. Para dar conta da dependência entre eles, podemos considerar que as componentes do momento de dipolo $\left(\mu_{i}\right)$ mudam ao modificar as componentes do campo elétrico $\left(\varepsilon_{j}\right)$ ao que é exposta a molécula mediante o tensor de polarizabilidade $\alpha_{i j}$ que é definido como:

$$
\mu_{i}=\sum_{j} \alpha_{i j} \varepsilon_{j}
$$

As componente de $\alpha_{i j}$ podem variar, da mesma maneira da que acontece com o dipolo elétrico, ao permitir a vibração do modo $Q_{i}$, mediante uma relação que pode ser aproximada da maneira:

$$
\alpha_{i j}=\alpha_{i j}^{0}+\sum_{k}\left(\frac{\partial \alpha_{i j}}{\partial Q_{k}}\right)_{0} Q_{k}+\cdots
$$

assim, o elemento de transição dipolar, desprezando termos de ordem superior em (3.17), é:

$$
\left(\boldsymbol{\mu}_{\nu_{k}^{\prime} \nu_{k}}\right)_{i}=\sum_{j} \varepsilon_{j}^{0}\left\{\left\langle\nu_{k}^{\prime}\left|\alpha_{i j}^{0}\right| \nu_{k}\right\rangle+\sum_{k}\left(\frac{\partial \alpha_{i j}}{\partial Q_{k}}\right)_{0}\left\langle\nu_{k}^{\prime}\left|Q_{k}\right| \nu_{k}\right\rangle\right\}
$$

O primeiro termo de (3.18), uma vez que não induze transições vibracionais (tem uma regra de seleção $\nu_{k}^{\prime}=\nu_{k}$ ), descreve uma reflexão do campo que perturba à molécula. Em termos de espectroscopia, é conhecido como espalhamento tipo Rayleigh.

Uma transição vibracional é Raman-ativa somente se a polarizabilidade varia enquanto os átomos da molécula são deslocados ao longo de uma coordenada normal $\left(\partial \boldsymbol{\alpha} / \partial Q_{k}\right)_{0} \neq 0$ 
se $\nu_{k}^{\prime}=\nu_{k}+1$ a transição é chamada de Stokes e se $\nu_{k}^{\prime}=\nu_{k}-1$, a transição é antiStokes. A frequência, do fóton necessário para excitar estas transições vibracionais, portanto, deverá ser a frequência fundamental $\omega_{k}$ definida na equação (3.10).

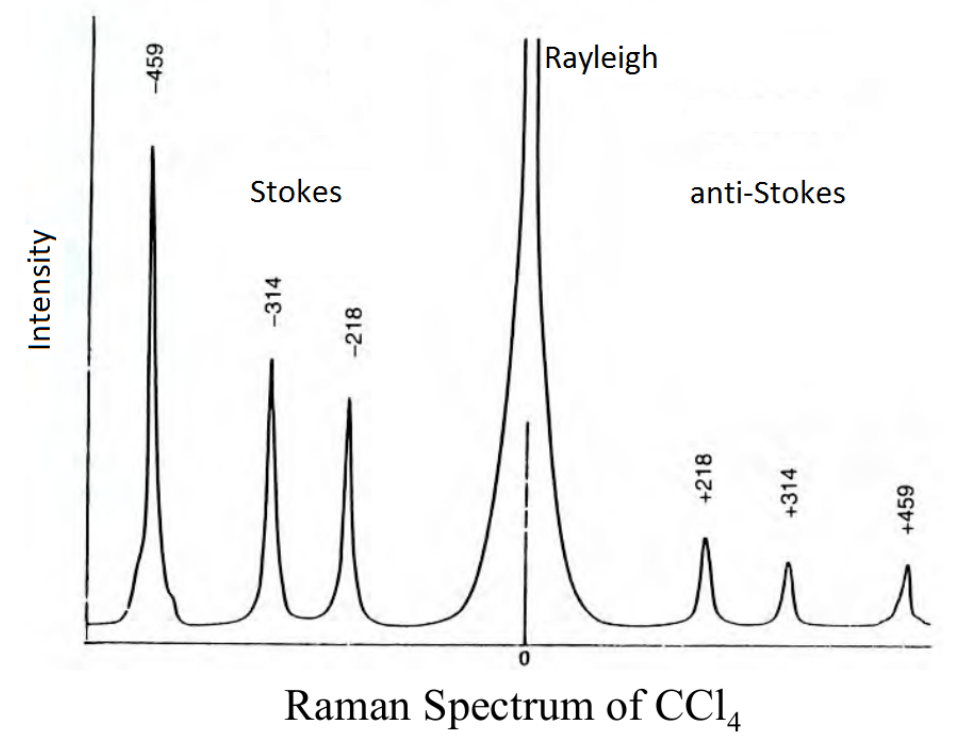

FiguRA 3.1: Espectro de radiação espalhada para uma molécula Raman-ativa em função da frequência[30], cada pico do espectro é a frequência do fotón espalhado no processo.

Experimentalmente, como é apresentado na figura 3.1, é encontrado que o espectro Raman de uma molécula consiste numa linha intensa, da frequência com que a molécula é excitada com mais linhas mais fracas localizadas simetricamente a cada lado da linha forte. As linhas de frequência menor do que a linha de excitação são as linhas de Stokes e as outras, são as linhas anti-Stokes.

A intensidade (em unidades CGS) da linha de excitação Raman é dada pela relação[32]

$$
I_{i}=\frac{2^{7} \pi^{5}}{3^{2} c^{4}} I_{0} N_{\omega_{i}}\left(\omega_{0}-\omega_{i}\right)^{4} \sum_{j, k}\left(\alpha_{j k}\right)^{2}
$$

onde $I_{0}$ e $\omega_{0}$ são, respetivamente, a intensidade e a frequência do raio de referência, $\omega_{i}$ é a frequência à qual acontece a transição do modo vibracional excitado (cuja frequência de transição é $\left.\left(\omega_{0}-\omega_{i}\right)\right)$, c é a velocidade da luz e $N_{\omega_{i}}$ é o numero de moléculas no estado vibracional, $a_{i j}$ corresponde a o elemento de matriz $\left\langle\nu_{k}^{\prime}\left|\alpha_{i} j\right| \nu_{k}\right\rangle$. Combinando as expressões (3.19) e (3.17), para transições tipo Stokes, é obtida:

$$
I_{i}=\frac{2^{7} \pi^{5}}{3^{2} c^{4}} I_{0} N_{\omega_{i}}\left(\omega_{0}-\omega_{i}\right)^{4}\left(\frac{1}{2 \omega_{i}}\right) \sum_{j, k}\left(\frac{\partial \alpha_{j k}}{\partial Q_{i}}\right)_{0}^{2}
$$


onde o termo $\left(1 / \omega_{i}\right)$ aparece a o expandir o operador $Q_{i}$ em operadores de criação de destruição, da maneira mostrada na equação (3.14). As derivadas da polarizabilidade $\partial \alpha / \partial Q_{i}$ formam um tensor de $3 \times 3 \times(3 N-6)$ componentes que é chamado de supertensor $\alpha_{Q}$. Com as componentes de esse tensor, são definidos os termos invariantes com respeito à orientação escolhida da molécula:

$$
\begin{aligned}
\alpha_{i}= & \frac{1}{3} \sum_{j} \frac{\partial \alpha_{j j}}{\partial Q_{i}} \\
\left(\beta_{i}\right)^{2}= & \frac{1}{2}\left[\left(\frac{\partial \alpha_{x x}}{\partial Q_{i}}-\frac{\partial \alpha_{y y}}{\partial Q_{i}}\right)^{2}+\left(\frac{\partial \alpha_{y y}}{\partial Q_{i}}-\frac{\partial \alpha_{z z}}{\partial Q_{i}}\right)^{2}+\left(\frac{\partial \alpha_{z z}}{\partial Q_{i}}-\frac{\partial \alpha_{x x}}{\partial Q_{i}}\right)^{2}\right] \\
& +3\left\{\left(\frac{\partial \alpha_{x y}}{\partial Q_{i}}\right)^{2}+\left(\frac{\partial \alpha_{x z}}{\partial Q_{i}}\right)^{2}+\left(\frac{\partial \alpha_{y z}}{\partial Q_{i}}\right)^{2}\right\}
\end{aligned}
$$

Com as quais é possível obter a Intensidade da linha Raman [33]

$$
I_{i}=\frac{2^{4} \pi^{5}}{45 c^{4}} I_{0} N_{\omega_{i}}\left(\omega_{0}-\omega_{i}\right)^{4}\left(\frac{1}{\omega_{i}}\right) g_{i}\left[45\left(\alpha_{i}\right)^{2}+7\left(\beta_{i}\right)^{2}\right]
$$

onde foi incluído o fator $g_{i}$ ou de degenerescência do modo vibracional. Com o propósito de trabalhar com uma quantidade independente de condições experimentais como $\omega_{0}, I_{0}$, usualmente é definida a chamada de intensidade padrão[34]

$$
S_{i}=g_{i}\left[45\left(\alpha_{i}\right)^{2}+7\left(\beta_{i}\right)^{2}\right]
$$

Além dela, também é útil a proporção de depolarização da linha vibracional:

$$
\rho_{i}=\frac{3\left(\beta_{i}\right)^{2}}{45\left(\alpha_{i}\right)^{2}+4\left(\beta_{i}\right)^{2}},
$$

que permite examinar a simetria das transições vibracionais. $\rho_{i}$ é nulo para transições vibracionais totalmente simétricas e toda vez que os elementos diagonais do tensor de derivadas da polarizabilidade $\left(\alpha_{i}\right)$ seja nulo, $\rho_{i}=0.75$. Combinando as equações (3.23) e (3.24) expressões para os valores absolutos de $\alpha_{i}$ e $\beta_{i}$ são obtidas:

$$
\begin{aligned}
\left|\alpha_{i}\right| & =\left[\frac{1}{45} \frac{S_{i}\left(3-4 \rho_{i}\right)}{3 g_{i}\left(1+\rho_{i}\right)}\right]^{1 / 2} \\
\left|\beta_{i}\right| & =\left[\frac{S_{i} \rho_{i}}{3 g_{i}\left(1+\rho_{i}\right)}\right]^{1 / 2}
\end{aligned}
$$

Finalmente o elemento de matriz $\alpha_{i}=\langle i|\alpha| 0\rangle$ (não confundir com os elementos diagonais do tensor de derivadas da polarizabilidade da equação (3.25)) em termos de $S_{i}$ e $\rho_{i}$ vai ser dado por:

$$
\alpha_{i}=\left[\frac{\hbar}{2 \omega_{i}} \frac{1}{45} \frac{S_{i}\left(3-4 \rho_{i}\right)}{3 g_{i}\left(1+\rho_{i}\right)}\right]^{1 / 2}
$$




\section{Capítulo 4}

\section{Formalismo de operadores de projeção de Feshbach e modelo de Gribakin-Lee}

\subsection{Introdução}

O formalismo de operadores de projeção de Feshbach (FPO), é o eixo central da atual teoria que descreve o processo de espalhamento pósitron-molécula [19, 20, 35]. Originalmente, o método foi criado para lidar com colisões nucleares mas possui um mecanismo geral que pode ser utilizado para qualquer processo de espalhamento onde se lide com estados metaestáveis acoplados a estados de espectro continuo além de transições entre os estados discretos, dentro do alvo, devidas à captura.

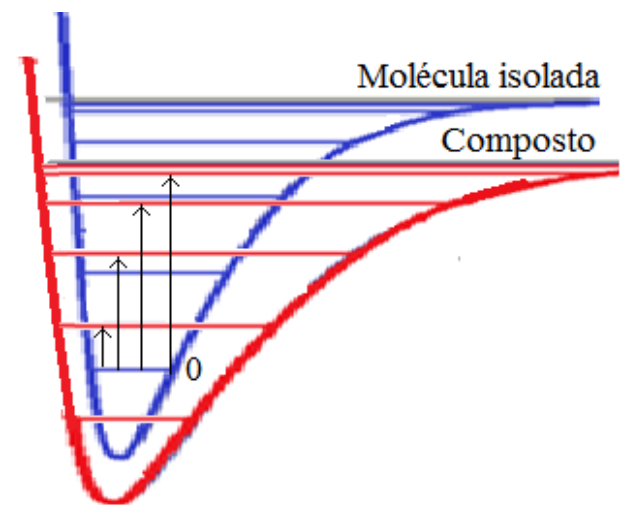

FigurA 4.1: Representação esquemática da ressonância vibracional de Feshbach. O estado Base da SEP da molécula isolada (linha azul) é acoplado com os estados exitados do composto pósitron molécula (linha vermelha). 
Qualitativamente, o formalismo é útil para descrever o pósitron, inicialmente num estado do contínuo (pósitron livre), acoplado com estados discretos do composto pósitronmolécula ocasionando um espalhamento ressonante. Com a energia excedente do acoplamento contínuo-discreto do pósitron, é feita uma transição vibracional desde o estado vibracional da molécula (canal vibracional de entrada) até um ou vários estados vibracionais do sistema pósitron-molécula. Em geral, esse estado vibracional é uma superposição de auto-estados do hamiltoniano associado à superfície de energia potencial (SEP) modificada pela presença do pósitron.

Um dos fatos ao levar em conta no método, é que que a excitação vibracional não é produzida diretamente pela interação do pósitron com os núcleos mas como uma consequência da correlação do pósitron com a estrutura eletrônica do alvo. Isto é consistente com o fato de que os elétrons vão responder consideravelmente mais rápido ao campo elétrico do pósitron do que os graus de liberdade nucleares. A transferência de energia, portanto, segue da diferença entre as superficeis de energia potencial do alvo isolado e do composto.

\subsection{Formalismo de operadores de projeção de Feshbach}

O formalismo teórico exposto nesta seção foi proposto em 2009[22], como uma adaptação do modelo original proposto por Feshbach [36]. Para nosso problema, o hamiltoniano do sistema pósitron-molécula pode ser escrito como

$$
H=T_{N}+T_{p}+H_{0}+V \equiv T_{N}+H_{e l e}
$$

onde $T_{N}$ e $T_{p}$ são as energias cinéticas dos núcleos e do pósitron, respetivamente, $V$ é o potencial de interação pósitron-molécula e $H_{0}$ é o hamiltoniano de interações internas do alvo mais a energia cinética dos elétrons. Os três últimos operadores, definem $H_{\text {ele }}$.

O formalismo está baseado na separação do espaço de Hilbert do pósitron em um (ou mais de um) estado discreto do composto $\left|\phi_{d}\right\rangle$ ortogonal a um espaço contínuo $\left\{\left|\phi_{k}\right\rangle\right\}$. O estado vibracional, será definido por um pacote de onda $|\xi\rangle$ dependente paramétricamente do autoestado eletrônico-positrônico. A definição dos operadores de projeção $Q=\left|\phi_{d}\right\rangle\left\langle\phi_{d}\right|$ (projeção sobre o espaço elétron-positrônico localizado) e $P=(1-Q)$, permite a decomposição do estado de espalhamento $\left(\left|\Psi_{\mathrm{k}}\right\rangle\right)$, asociado ao pósitron incidente com número de onda $\mathrm{k}$, de acordo com $\left|\Psi_{\mathrm{k}}\right\rangle=\left|\Psi_{P}\right\rangle+\left|\Psi_{Q}\right\rangle$. Assim, a equação de Schrödinger pode ser escrita da maneira:

$$
H(P+Q)\left|\Psi_{\mathrm{k}}\right\rangle=E\left|\Psi_{\mathrm{k}}\right\rangle
$$


Uma vez que os operadores de projeção são definidos no espaço estritamente eletrônicopositrônico, observamos que $Q H P=Q H_{\text {ele }} P$ pois $\left[T_{N}, Q\right]=\left[T_{N}, P\right]=0$. Portanto, projetando sobre os espaços $P$ e $Q$ obtemos (usando $P Q=0$ e $P^{2}=P$ ):

$$
\begin{aligned}
& P(E-H) P\left|\Psi_{P}\right\rangle=P H Q\left|\Psi_{Q}\right\rangle \\
& Q(E-H) Q\left|\Psi_{Q}\right\rangle=Q H P\left|\Psi_{P}\right\rangle=Q H_{\text {ele }} P\left|\Psi_{P}\right\rangle
\end{aligned}
$$

Com a ideia de justificar passos seguintes, é observado que pelo menos no estado deslocalizado do pósitron, a correlação elétron-pósitron pode ser aproximada a um campo médio. Dessa maneira, é posível integrar nas coordenadas do pósitron de maneira separada à integração nas coordenadas do elétron. Assim, o estado $\left|\phi_{\mathrm{d}}\right\rangle$ é decomposto na parte eletrônica $\left|\phi_{\mathrm{d}}^{\text {ele }}\right\rangle$ e a do pósitron $\left|\phi_{\mathrm{d}}^{p}\right\rangle$. Assim:

$$
\left|\Psi_{Q}\right\rangle \approx|\xi\rangle\left|\phi_{d}^{e l e}\right\rangle\left|\phi_{d}^{p}\right\rangle \quad\left|\Psi_{P}\right\rangle \approx\left|\eta_{\nu}\right\rangle\left|\phi_{0}^{e l e}\right\rangle\left|\phi_{\mathbf{k}}\right\rangle
$$

Uma vez que o estado vibracional depende paramétricamente do estado eletron-positrônico, é uma aproximação razoavel que, se o pósitron ficar num estado deslocalizado, o movimento dos núcleos é descrito pelos autoestados vibracionais da molécula isolada $\left|\eta_{\nu}\right\rangle$.

Com essas suposições, obtemos as soluções às equações (4.3) que definem as funçõess $\left|\Psi_{P}\right\rangle$ e $\left|\Psi_{Q}\right\rangle[36],[37],[38]$

$$
\begin{aligned}
\left|\Psi_{Q}\right\rangle & =\frac{1}{Q(E-H) Q} Q H_{\text {ele }} P\left|\Psi_{P}\right\rangle \\
\left|\Psi_{P}\right\rangle & =\left|\eta_{0}\right\rangle\left|\phi_{0}^{\text {ele }}\right\rangle\left|\phi_{\mathbf{k}}\right\rangle+\frac{1}{P(E-H) P} P H_{\text {ele }} Q\left|\Psi_{Q}\right\rangle
\end{aligned}
$$

de maneira que $\left|\phi_{0}^{\text {ele }}\right\rangle\left|\phi_{\mathbf{k}}\right\rangle$ é um auto estado de $P\left(H_{\text {ele }}\right) P$. Substituindo a equação (4.7) em (4.4) encontramos:

$$
Q(E-H) Q\left|\Psi_{Q}\right\rangle=Q H_{\text {ele }} P\left(\left|\eta_{0}\right\rangle\left|\phi_{0}^{\text {ele }}\right\rangle\left|\phi_{\mathbf{k}}\right\rangle+G_{P}\left(P H_{\text {ele }} Q\right)\left|\Psi_{Q}\right\rangle\right)
$$

onde definimos $G_{P}=[P(E-H) P]^{-1}$.

Decompondo a função de onda total da maneira feita na equação (4.5) e projetando (4.8) sobre o estado discreto eletrônico e do pósitron $\left\langle\phi_{\mathrm{d}}\right|=\left\langle\phi_{\mathrm{d}}^{\mathrm{p}}\right|\left\langle\phi_{\mathrm{d}}^{\text {ele }}\right|$ e utilizando $\left\langle\phi_{\mathrm{d}} \mid \phi_{\mathrm{d}}\right\rangle=1$ e $\left\langle\phi_{\mathrm{d}}\right| Q=\left\langle\phi_{\mathrm{d}}\right|$ obtemos a expressão:

$$
\left[E-\varepsilon_{d}-V_{0}-T_{N}\right]|\xi\rangle=U_{\mathbf{k}}\left|\eta_{0}\right\rangle+\left\langle\phi_{d}\left|H_{e l e} P G_{P} P H_{\text {ele }}\right| \phi_{d}\right\rangle|\xi\rangle
$$

Aqui, foi expresso o operador $Q(E-H) Q=E-\varepsilon_{d}-V_{0}$, onde $V_{0}$ é o operador, que, na representação das coordenadas $\{\mathbf{R}\}$ é a SEP do alvo isolado $V_{0}(\mathbf{R})$ e $\varepsilon_{d}$ um operador 
visto como os efeitos da correlação elétron-pósitron definido pela equação:

$$
\varepsilon_{d}=\left\langle\phi_{\mathrm{d}}\left|H_{e l e}\right| \phi_{\mathrm{d}}\right\rangle-V_{0}
$$

Vemos que aqui, uma vez não e feita a integração eletrõnica de maneira separada com a positrônica, não é feita a aproximação de campo médio (4.5). Na equação (4.9) também é definida a amplitude de acoplamento $\left(U_{\mathbf{k}}\right)$ para o acoplamento entre o espaço discreto e o contínuo,

$$
U_{\mathbf{k}_{i}}=\left\langle\phi_{\mathrm{d}}^{\mathrm{p}}\left|\left\langle\phi_{\mathrm{d}}^{\text {ele }}\left|Q H_{\text {ele }} P\right| \phi_{0}^{\text {ele }}\right\rangle\right| \phi_{\mathbf{k}_{i}}\right\rangle=\left\langle\phi_{\mathrm{d}}^{\mathrm{p}}\left|V_{\text {cap }}\right| \phi_{\mathbf{k}_{i}}\right\rangle
$$

onde $V_{\text {cap }}=\left\langle\phi_{\mathrm{d}}^{\text {ele }}\left|Q H_{\text {ele }} P\right| \phi_{0}^{\text {ele }}\right\rangle=\left\langle\phi_{\mathrm{d}}^{\text {ele }}\left|H_{\text {ele }}\right| \phi_{0}^{\text {ele }}\right\rangle$ é o elemento de matriz integrado só nas coordenadas eletrônicas (sem o pósitron). É dependente parametricamente das coordenadas nucleares e diretamente das coordenadas do pósitron. A definição de $V_{\text {cap }}$, portanto, é equivalente a supor que correlação elétron-pósitron satisfaz uma aproximação de campo medio. Esta aproximação é util para acoplar o estado discreto com o continuo uma vez que a correlação é pequena no estado deslocalizado.

A representação de $G_{P}$ na base dos auto estados de $P H P$, depois ao integrar nas coordenadas eletrônicas, é:

$$
\begin{aligned}
G_{P} & =\lim _{\epsilon \rightarrow 0} \int d \mathbf{k} \sum_{\nu} \frac{|\mathbf{k}\rangle\left|\eta_{\nu}\right\rangle\left\langle\eta_{\nu}\right|\langle\mathbf{k}|}{E-\frac{k^{2}}{2}-\omega_{\nu}+i \epsilon} \\
& =\wp \int d \mathbf{k} \sum_{\nu} \frac{|\mathbf{k}\rangle\left|\eta_{\nu}\right\rangle\left\langle\eta_{\nu}\right|\langle\mathbf{k}|}{E-\frac{k^{2}}{2}-\omega_{\nu}}-\pi i \int d \mathbf{k} \sum_{\nu}|\mathbf{k}\rangle\left|\eta_{\nu}\right\rangle\left\langle\eta_{\nu}\right|\langle\mathbf{k}| \delta\left(E-\frac{k^{2}}{2}-\omega_{\nu}\right)
\end{aligned}
$$

onde $\wp$ é o valor principal de Cauchy, enquanto $\delta(x)$ é a distribuição de Dirac. Aquí, tomamos como referencia, a energia do estado base vibracional, de maneira que $\left(V_{0}(\mathbf{R})+\right.$ $\left.T_{N}\right)\left|\eta_{\nu}\right\rangle=\omega_{\nu}\left|\eta_{\nu}\right\rangle$. Assim, ao integrar nas coordenadas do pósitron e utilizando o operador $U_{\mathbf{k}}$ definido pela equação (4.11), obtemos:

$$
\left\langle\phi_{d}^{p}\left|H_{e l e} P G_{P} P H_{e l e}\right| \phi_{d}^{p}\right\rangle=\Delta-\frac{i}{2} \Gamma
$$

onde:

$$
\Gamma(E)=2 \pi \int d \mathbf{k} \sum_{\nu} U_{\mathbf{k}}^{*}\left|\eta_{\nu}\right\rangle\left\langle\eta_{\nu}\right| U_{\mathbf{k}} \delta\left(E-\frac{k^{2}}{2}-\omega_{\nu}\right)
$$

e

$$
\Delta(E)=\frac{1}{2 \pi} \wp \int d \mathbf{k} \sum_{\nu} \frac{\Gamma(E)}{\left(E-\frac{k^{2}}{2}-\omega_{\nu}\right)}
$$

operadores na base das vibrações $\left(\left\{\eta_{\nu}\right\}\right)$ e que correspondem a operadores não locais na representação das coordenadas uma vez que projetam os estados vibracionais nos estados do alvo isolado. 
Dessa maneira, obtemos a expressão dependente da energia para $|\xi\rangle$

$$
\left[E-\varepsilon_{d}-V_{0}-T_{N}-\Delta(E)+\frac{i}{2} \Gamma(E)\right]|\xi\rangle=U_{\mathbf{k}}\left|\eta_{0}\right\rangle
$$

assim, é possível definir:

$$
|\xi\rangle=\frac{U_{\mathbf{k}}}{\left[E-T_{N}-V_{o p t}\right]}\left|\eta_{0}\right\rangle
$$

onde o operador $V_{o p t}=\varepsilon_{d}+V_{0}+\Delta-\frac{i}{2} \Gamma$. Vemos que existe uma similitude na forma de (4.17) com (2.14). A inclusão graus internos do alvo, na teoria de Feshbach, faz que o fator $\left\langle\phi_{d} \mid \psi\right\rangle$ em principio dependente da energia do estado positrônico, vire um operador no espaco vibracional.

\subsubsection{Cálculo de $Z_{\text {eff. Aproximações úteis }}$}

Uma vez que a função de onda do sistema no espalhamento pode ser expressa como a soma de duas funções: $\left|\Psi_{\mathbf{k}_{i}}\right\rangle=\left|\Psi_{P}\right\rangle+\left|\Psi_{Q}\right\rangle$, ao momento de calcular $Z_{\text {eff }}$, obtemos:

$$
\begin{aligned}
Z_{\mathrm{eff}} & =\left\langle\Psi_{k_{i}}\left|\sum_{j=1}^{Z} \delta\left(\mathbf{r}_{j}-\mathbf{r}_{p}\right)\right| \Psi_{k_{i}}\right\rangle \\
& =\left\langle\Psi_{Q}\left|\sum_{j=1}^{Z} \delta\left(\mathbf{r}_{j}-\mathbf{r}_{p}\right)\right| \Psi_{Q}\right\rangle+\left\langle\Psi_{P}\left|\sum_{j=1}^{Z} \delta\left(\mathbf{r}_{j}-\mathbf{r}_{p}\right)\right| \Psi_{P}\right\rangle+\operatorname{Re}\left[\left\langle\Psi_{Q}\left|\sum_{j=1}^{Z} \delta\left(\mathbf{r}_{j}-\mathbf{r}_{p}\right)\right| \Psi_{P}\right\rangle\right]
\end{aligned}
$$

Espera-se que o pósitron no contínuo (na base de auto-estados de PHP) tenha uma densidade de probabilidade muito pequena na vizinhança do alvo e seja acoplado fracamente com o movimento nuclear. Como consequência, as contribuições dos termos $P P$ e $Q P$ na equação (4.18) deveriam ser desprezíveis em relação ao termo $Q Q$ que descreve ao pósitron ligado. Dessa maneira podemos considerar que a aniquilação somente ocorre no estado discreto. Com a ajuda das equações (4.5) e (4.17), a componente $Q$ da função de onda pode ser escrita como

$$
\left|\Psi_{Q}\right\rangle=\left|\phi_{d}\right\rangle|\xi\rangle=\left|\phi_{d}\right\rangle \frac{1}{E-T_{N}-V_{o p t}} U_{\mathbf{k}}\left|\eta_{0}\right\rangle
$$

de maneira que pode ser feita, sem um erro apreciável, a aproximação:

$$
\begin{aligned}
Z_{\mathrm{eff}}(k) & \approx\left\langle\Psi_{Q}\left|\sum_{j=1}^{Z} \delta\left(\mathbf{r}_{j}-\mathbf{r}_{p}\right)\right| \Psi_{Q}\right\rangle \\
& =\left\langle\eta_{0}\left|U_{\mathbf{k}}^{*} \frac{1}{\left[E-T_{N}-V_{o p t}\right]^{\dagger}} \rho_{d} \frac{1}{\left[E-T_{N}-V_{o p t}\right]} U_{\mathbf{k}}\right| \eta_{0}\right\rangle
\end{aligned}
$$


com

$$
\rho_{d}=\left\langle\phi_{\mathrm{d}}\left|\sum_{j=1}^{Z} \delta\left(\mathbf{r}_{j}-\mathbf{r}_{p}\right)\right| \phi_{\mathrm{d}}\right\rangle
$$

entendida como a densidade eletrônica na posição do pósitron no estado discreto e que, em geral, é operador do espaço vibracional. Uma simplificação considerável pode ser obtida se $\rho_{d}$ é fracamente dependente das coordenadas nucleares ou seja, que o overlap entre as densidades de probabilidade dos elétrons e do pósitron não muda muito ao mudar a geometria da molécula e pode ser tomado:

$$
\left\langle\eta_{\nu}\left|\rho_{d}\right| \eta_{\nu}^{\prime}\right\rangle \simeq \rho_{d} \delta_{\nu, \nu^{\prime}}
$$

onde, por exemplo, pode-se tomar $\rho_{d}$ como o overlap na geometria de equilíbrio. Dessa maneira, introduzindo algumas relações de completeza, a partir de (4.20), pode ser obtido:

$Z_{\mathrm{eff}}(E)=\rho_{d} \sum_{\nu, \nu^{\prime}, \nu^{\prime \prime}}\left\langle\eta_{0}\left|U_{\mathbf{k}}^{*}\right| \eta_{\nu}\right\rangle\left\langle\eta_{\nu}\left|\frac{1}{\left[E-T_{N}-V_{o p t}\right]^{\dagger}}\right| \eta_{\nu^{\prime}}\right\rangle\left\langle\eta_{\nu^{\prime}}\left|\frac{1}{\left[E-T_{N}-V_{o p t}\right]}\right| \eta_{\nu^{\prime \prime}}\right\rangle\left\langle\eta_{\nu^{\prime \prime}}\left|U_{\mathbf{k}}\right| \eta_{0}\right\rangle$

Um dos principais problemas com que é preciso lidar é com a diagonalização do operador $\left[E-T_{N}-V_{o p t}\right]$. Para resolve-o numericamente é preciso expressa-lo numa base conveniente. A aproximação local, permite que $\Delta$ e $\Gamma$ sejam aproximados a operadores constantes considerando que não mudam muito com os estados vibracionais da molécula de maneira que $T_{N}+V_{\text {opt }}$ é diagonal na base dos autoestados de $T_{N}+V_{0}+\varepsilon_{d}$.

Uma maneria de faze-lo é restringindo o canal de entrada a o estado fundamental e considerando que o operador $U_{\mathbf{k}_{\nu}}$ induz somente transições de primeira ordem $(0 \rightarrow 1)$ no estado $\nu$. Assim, é possível fazer uma estimativa de $\Gamma$ dependendo da transição vibracional induzida no acoplamento.

$$
\Gamma(E) \approx \sum_{\nu} \Gamma_{\nu}^{e}=2 \pi \sum_{\nu} \int d \mathbf{k}\left|U_{\mathbf{k}, \nu}\right|^{2} \delta\left(k^{2} / 2-\varepsilon_{\nu}\right)
$$

onde $U_{\mathbf{k}, \nu}=\left\langle\eta_{\nu}\left|U_{\mathbf{k}}\right| \eta_{0}\right\rangle$ e $\varepsilon_{\nu}$ é a energia de transição vibracional. O operador $\Delta$, por outro lado, pode ser aproximado a um operador dependente únicamente das coordenadas nucleares e incluido na superficie de energia potencial do composto de maneira que, indistinguiblemente o operador $\varepsilon_{d}$ é modificado implícitamente a partir de (4.10):

$$
\varepsilon_{d}=\left\langle\phi_{\mathrm{d}}\left|H_{e l e}\right| \phi_{\mathrm{d}}\right\rangle+\Delta-V_{0} .
$$

Uma vez que não será objetivo da pesquisa fazer estimativas de $\Delta$, vamos supor ele como un operador constante que também não poderemos estimar. Assim, aproximaremos 
(4.25) a (4.10). Estas aproximações poderão será estudadas mais profundamente no modelo Gribakin-Lee, apresentado na seção seguinte.

\subsection{Modelo de Gribakin-Lee}

Um caso particular do modelo de operadores de projeção de Feshbach (FPO) é o modelo de Gribakin-Lee (GL), cuja fundamentação teórica, essencialmente fenomenológica, foi publicada em 2006 [39]. O modelo é um caso particular do FPO. Os resultados do modelo, foram comparados com o espectro de Halometanos $\mathrm{CH}_{3} \mathrm{~F}$ [40], $\mathrm{CH}_{3} \mathrm{Cl}$ e $\mathrm{CH}_{3} \mathrm{Br}$ [41], moléculas que são ideais ao apresentar todas as suas transições no infravermelho.[39]

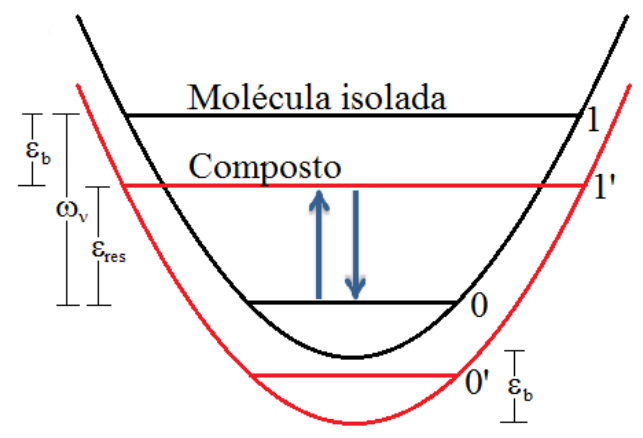

Figura 4.2: Representação esquemática das aproximações feitas nas SEP da molécula isolada (linha preta) e a SEP do composto pósitron molécula (linha vermelha).

A premissa do modelo é tomar constante e independente da geometria do composto o operador $\varepsilon_{d}$, definido em (4.10), de maneira que a molécula e o composto compartem os mesmos estados vibracionais mas com um espectro de energia deslocado um valor da energia $\varepsilon_{b}<0$ como é apresentado na figura 4.2, de maneira que as transições vibracionais do estado fundamental até o primeiro estado excitado do modo $\nu$ não precissam energia $\omega_{\nu}$ mas uma energia menor $\omega_{\nu}^{\prime}=\omega_{\nu}+\varepsilon_{b}[39]$. Portanto, a posição $\varepsilon_{\mathrm{r}}$ do pico de uma ressonância em $Z_{\text {eff }}$, como foi antecipado na figura 1.1, vai estar deslocada uma quantidade $\varepsilon_{b}$ com respeito à energia de transição vibracional da molécula isolada $\omega_{\nu}$ seguindo a relação:

$$
\varepsilon_{\mathrm{r}}=\omega_{\nu}+\varepsilon_{b}
$$

Dessa forma, medindo a posição dos picos do espectro de $Z_{\text {eff }}$ e comparando às respectivas posições no espectro vibracional da molécula, poderemos obter o único parâmetro livre na teoria: a energia de ligação do pósitron $\left(\varepsilon_{b}\right)$. O procedimento necessário para comparar as previsões teóricas com os resultados experimentais, é detalhado no apêndice C.3. 
Uma vez que como foi explicado na seção anterior, é tomada a aproximação de que só são importantes as transições vibracionais de primeira ordem, o canal de saída será o mesmo de entrada, de maneira que, na equação (4.23),

$$
\left\langle\eta_{0}\left|U_{\mathbf{k}}^{*}\right| \eta_{\nu^{\prime}}\right\rangle\left\langle\eta_{\nu^{\prime}}\left|U_{\mathbf{k}}\right| \eta_{0}\right\rangle=\left|U_{\mathbf{k}, \nu}\right|^{2}
$$

É incluído o efeito do operador $\Delta$ na definição de $\varepsilon_{b}$ de maneira que $V_{\mathrm{opt}} \approx V_{0}+\varepsilon_{b}+\Delta-$ $(i / 2) \Gamma$ com $V_{0}$ a SEP da molécula isolada, sendo $\Gamma(E)=\sum_{\nu} \Gamma_{\nu}$ onde cada operador $\Gamma_{\nu}$ que atua somente num modo vibracional $\nu$. Seguindo seguindo o formalismo apresentado no apêndice C.3, $\Gamma_{\nu}$ fica:

$$
\Gamma_{\nu}^{e}=2 \pi \int\left|U_{\mathbf{k}, \nu}\right|^{2} \delta\left(k^{2} / 2-\omega_{\nu}-\varepsilon_{b}\right) d \mathbf{k}=8 \pi^{2} k_{\nu}\left|U_{k, \nu}\right|^{2} ; \quad k_{\nu}=\sqrt{2\left(\omega_{\nu}+\varepsilon_{b}\right)}
$$

onde $U_{k}$ é a média angular de $U_{\mathbf{k}}$ e vai depender da forma do potencial de captura $V_{\text {cap }}$ que tomemos para acoplamento contínuo-discreto do pósitron pela equação (4.11). No modelo GL, é tomado $V_{\text {cap }}(\mathrm{r})=-\boldsymbol{\mu} \cdot \hat{\mathbf{r}} / r^{2}$.

Com essas aproximações, pode-se escrever:

$$
\sum_{\nu^{\prime}}\left\langle\eta_{\nu}\left|\frac{1}{\left[E-T_{N}-V_{o p t}\right]^{\dagger}}\right| \eta_{\nu^{\prime}}\right\rangle\left\langle\eta_{\nu^{\prime}}\left|\frac{1}{\left[E-T_{N}-V_{o p t}\right]}\right| \eta_{\nu}\right\rangle \approx \frac{1}{\left(k^{2} / 2-\omega_{\nu}-\bar{\varepsilon}_{b}\right)^{2}+\Gamma_{\nu}^{2} / 4}(4 .
$$

aqui $\bar{\varepsilon}_{b}=\varepsilon_{b}+\Delta$. Gribakin-Lee não leva em conta o termo $\Delta$, no entanto, em termos estritos, no experimento é medido $\bar{\varepsilon}_{b}$ e não tem maneira simples de distinguir $\varepsilon_{b}$ de $\Delta$.

Finalmente, depois de fazer a soma sob todas a transições vibracionais, seguindo a fórmula (4.23) $\{\nu\}$ e expressando $U_{\mathbf{k}, \nu}$ em termos de $\Gamma_{\nu}^{e}$ pela equação (4.28):

$$
Z_{\mathrm{eff}}=\rho_{d} \sum_{\nu} \frac{2 \pi}{k_{\nu}} \frac{g_{\nu} \Gamma_{\nu}^{e}}{\left(k^{2} / 2-\omega_{\nu}-\bar{\varepsilon}_{b}\right)+\left(\Gamma_{\nu}^{e} / 4\right)^{2}}
$$

onde, $g_{\nu}$ é a degenerescência do $\nu$-ésimo modo vibracional. O termo $\rho_{d}$, é definido na equação (4.21) e calculado utilizando, como chute, a função de onda localizada do pósitron $\left\langle\mathbf{r} \mid \phi_{d}^{p}\right\rangle=A e^{-\kappa r} / r \operatorname{com} \kappa=\sqrt{2 \varepsilon_{b}}$. 


\section{Capítulo 5}

\section{O acoplamento pósitron-molécula para vibrações com atividade Raman}

\section{$5.1 \quad$ Antecedentes}

Na proposta original do modelo de Gribakin-Lee, uma vez que o acoplamento contínuo=discreto do pósitron é intermediado por um potencial dipolar, somente justifica o acoplamento em transições vibracionais ativas no infravermelho (IR-ativas)[39].

Utilizando esse modelo para transições vibracionais IR-ativas, a estimativa de $\Gamma_{\nu}^{e}$, da maneira exposta no apêndice $\mathrm{C}$, permite prever uma relação importante entre $\Gamma_{\nu}^{e}, \Gamma^{a}$ (a largura de aniquilação direta pósitron-elétron) e $\sigma$, a resolução em energia do feixe de pósitrons utilizado nos experimentos[39]:

$$
\Gamma^{a} \ll \Gamma_{\nu}^{e} \ll \sigma
$$

Primeiramente, sendo $\Gamma^{a} \ll \Gamma_{\nu}^{e}$, a largura de decaimento vai ser principalmente a do acoplamento pósitron-molécula $\Gamma_{\nu}^{e}$ de maneira que a largura total de aniquilação $\left(\Gamma_{\nu}\right)$ pode ser aproximada por $\Gamma_{\nu}=\Gamma^{a}+\Gamma_{\nu}^{e} \approx \Gamma_{\nu}^{e}$. Por outro lado, o fato de, $\sigma$ ser muito maior a $\Gamma_{\nu}^{e}$ permite fazer a aproximação $\Gamma_{\nu}^{e} \rightarrow 0$ da maneira exposta no apêndice C e o espectro de $Z_{\text {eff }}$ composto por perfis de Breit-Wigner, pode ser aproximado, na equação (C.11), por somas de deltas de Dirac em cada ressonância, obtendo, depois da convolução sobre a distribuição em energia do feixe de pósitrons utilizado nos experimentos, um $\bar{Z}_{\text {eff }}$ simples:

$$
\bar{Z}_{\mathrm{eff}}(\epsilon)=2 \pi^{2} \rho_{\mathrm{ep}} \sum_{\nu} \frac{g_{\nu} \Gamma_{\nu}^{e}}{k_{\nu} \Gamma_{\nu}} \Delta\left(\epsilon-\varepsilon_{\nu}\right) \approx 2 \pi^{2} \rho_{\mathrm{ep}} \sum_{\nu} \frac{g_{\nu}}{k_{\nu}} \Delta\left(\epsilon-\varepsilon_{\nu}\right)
$$


A equação (5.2) é explicada no apêndice $C$ que, ao não depender, explicitamente de $\Gamma_{\nu}^{e}$, perde toda a informação do potencial de acoplamento $V_{\text {cap }}$ de maneira que o valor exato de $\Gamma_{\nu}^{e}$ não é importante, desde que seja garantida a relação (5.1), interessando simplesmente a posição $\left(\varepsilon_{\nu}\right)$ dos picos de ressonância. Sob a premissa de que (5.1) é válida para qualquer transição vibracional, a fórmula (5.2) tem sido empregada em transições não IR-ativas[12].

No entanto, mesmo que foi observado que existem picos no espectro de $Z_{\text {eff }}$ associados a transições Raman-ativas uma vez que emcontram-se fracamente deslocados dos picos dessas transições, da maneira apresentada, por exemplo na figura 5.1a, não existem cálculos que permitam justificar, para eles, o limite (5.1) nem também existe um procedimento para estimar um tempo de vida do estado meta-estável associado a cada excitação vibracional. O propósito deste capítulo será, portanto, apresentar um conjunto de ferramentas teórico-computacionais adequadas para fazer uma estimativa de $\Gamma_{\nu}^{e}$ dando continuidade à linha de pesquisa desenvolvida pelo grupo.[23]

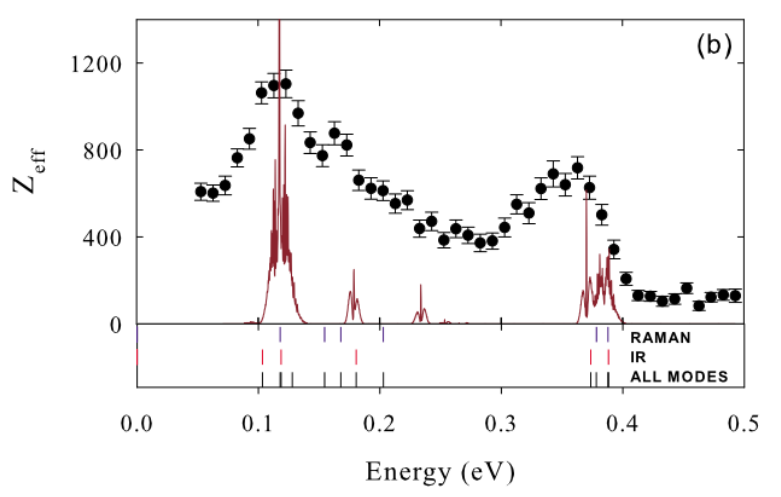

(A)

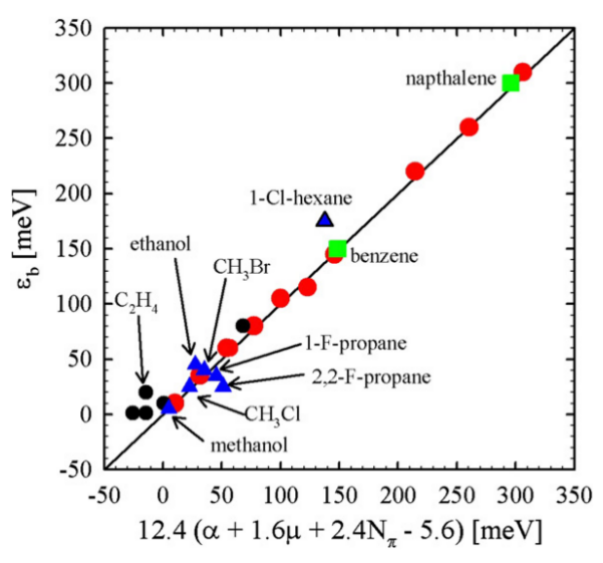

(B)

FigurA 5.1: (a) Comparação de $Z_{\text {eff }}(\bullet)$ e o espectro vibracional do Etileno $\left(\mathrm{C}_{2} \mathrm{H}_{4}\right)$ (linha continua) sugerindo uma energia de ligação $\varepsilon_{b} \approx 10 \mathrm{meV}$ (b) Ajuste da energia de ligação como é descrita na equação (5.3) usando a polarizabilidade $(\alpha)$, o momento de dipolo permanente $(\mu)$ e o numero de ligações $\pi\left(N_{\pi}\right)$ para diferentes moléculas[43].

A partir dos valores experimentais para $\varepsilon_{b}$, entendidos como o deslocamento dos picos de $Z_{\text {eff }}$ do espectro vibracional, obtidos para alcanos, moléculas aromáticas e álcoois assim como hidrocarbonetos parcialmente halogenados (com momento de dipolo permanente), alquenos, alquenos perhalogenados e alquenos parcialmente fluorionados, foi sugerida uma relação linear entre a energia de ligação $\left(\varepsilon_{b}\right)$, a polarizabilidade média $(\alpha)$, o momento de dipolo $(\mu)$ e a quantidade de ligações $\pi\left(N_{\pi}\right)$ da molécula, descrita pela equação[43]:

$$
\varepsilon_{b}=12.4\left(\alpha+16 \mu+2.4 N_{\pi}-5.6\right)(\mathrm{meV})
$$


com $\alpha$ em unidades de $\AA^{3}, \mu$ em unidades de $D$ e cujo ajuste é apresentado na figura 5.1b. Esta fórmula, empírica, permitiu fazer algumas previsões para algumas moléculas pequenas.[43]

Embora a equação (5.3) tem sido criticada ao incluir aparâmwetros equivalentes $\alpha$ e $N_{\pi}$ que em principio estão relacionado, uma das suas qualidades é admitir que o pósitron pode ser ligado pela correlação pósitron-elétron implícita na polarizabilidade média $\alpha$, possibilidade não contemplada pelo potencial $-\boldsymbol{\mu} \cdot \hat{\mathbf{r}} / r^{2}$. Dessa maneira, propomos aqui, um mecanismo de acoplamento pósitron-molécula via um potencial proporcional a $\alpha$ com a forma $V_{\text {cap }}(r, \mathbf{R})=-\alpha(\mathbf{R}) / 2 r^{4}$, onde $r$ é a distância ao centro da molécula.

Já foi previsto que a correlação pósitron-molécula pode modificar a polarizabilidade da molécula[44], de maneira que, se o potencial depende diretamente de $\alpha$ será possível o acoplamento do pósitron induzindo excitações vibracionais Raman-Ativas da maneira prevista na seção 3.2.2. Uma vez que $V_{\text {cap }}$ depende diretamente de uma propriedade puramente eletrônica como a polarizabilidade, o acoplamento pósitron-molécula mediado por este tipo de potencial justifica, também, a deformação da nuvem eletrônica da molécula que, como será apresentado na próxima seção, será vital para descrever a dinâmica do composto pósitron-molécula.

A ideia vem do modelo proposto em 2012 na referência[45], no qual a ligação do pósitron a uma molécula não polar, pode ser descrito por meio do potencial regularizado e esfericamente simétrico:

$$
V=V_{h c}-\alpha / 2 r^{4}, \quad V_{h c}=\left[\begin{array}{cc}
\infty & r<r_{0} \\
0 & r>r_{0}
\end{array}\right]
$$

onde $r$ é a distância do pósitron ao centro da molécula, $r_{0}$ é um raio de corte ajustável que regulariza o potencial no origem. A escolha de $V_{h c}$ é a regularização mais simples e que evita o problema que tem o potencial $V_{c a p}=-\alpha / 2 r^{4}$ de não ser auto-adjunto[45].

O único parâmetro livre no potencial (5.4) é o raio de corte $\left(r_{0}\right)$. Para alguns valores de polarizabilidade $(\alpha)$, ele foi modificado de maneira que a energia do estado ligado do Hamiltoniano associado, calculada com o programa FGHEVEN[46] baseado no método numérico de Hamiltoniano de grelha de Fourier [47], coincidisse com a energia de ligação medida nos experimentos[48]. Os valores de $r_{0}$ obtidos para diferentes valores de polarizabilidade $(\alpha)$ permitiram ajustar, uma relação polinomial que prevê o raio de corte $\left(r_{0}\right)$ em $(\AA)$ e em função de $\alpha[45]$ :

$$
r_{0}=0.6293+\left(7687 \alpha-197.6 \alpha^{2}+1.228 \alpha^{3}\right) \times 10^{-5}
$$

valida no intervalo $\alpha \in(5.6,30.3) \AA^{3}$ 
Neste capítulo, será proposto um formalismo teórico similar ao de Gribakin-Lee que consegue descrever o acoplamento pósitron-molécula para transições vibracionais Ramanativas utilizando um potencial de acoplamento do tipo (5.4) obtendo larguras de ressonância $\Gamma_{\nu}^{e}$ com ordens de grandeza que justificam a aproximação (5.1) para esse tipo de modos.

Experimentalmente, a contribuição tem muita importância pois, atualmente, não existem muitos tipos de moléculas onde a teoria de Gribrakin-Lee e o experimento possam ser comparados. Nos principais experimentos de aniquilação positrônica, a maior parte das moléculas que têm sido medidas, são fracamente polares ou não polares como, por exemplo, alcanos $\left(C_{n} H_{2 n+2}\right)$ [43], ou um espectro rico em transições não ativas no IR como álcoois ou benzeno. Um modelo que permita incluir excitações vibracionais Raman-ativas baseado no potencial (5.4) permitindo uma verificação da relação (5.1), seria um passo importante na descrição da aniquilação ressonante[20].

\subsection{Detalhes do modelo}

Nossa proposta inicial para a estimativa das larguras de acoplamento para modos Ramanativos, foi buscar um modelo analítico similar a o modelo de Gribakin-Lee. Além disso, foi possível contar com o código do programa FGHEVEN[46] utilizado para obter as autofunções da equação de Schrödinger associada ao potencial (5.4). Assim, foi possível abordar o problema, conjuntamente, desde o ponto de vista analítico e o computacional. Abaixo, será presentada uma comparação entre estas duas aproximações.

\subsubsection{Cálculo da amplitude de captura}

Partindo da teoria geral de FPO, aproximaremos o estado ligado do pósitron $\phi_{d}$ à solução do Hamiltoniano associado ao potencial de acoplamento (5.4) e o estado de espalhamento $\phi_{\mathbf{k}} \approx e^{i \mathbf{k} \mathbf{r}} /(2 \pi)^{3 / 2}$ acoplados pelo potencial de captura (5.4). Uma vez que uma solução analítica exata de (5.4) não é facilmente obtida, foi proposta uma função tentativa:

$$
\phi_{d}(r)=\left(\frac{4 \gamma^{5}}{3+3 \gamma r_{0}+\left(\gamma r_{0}\right)^{2}}\right)^{1 / 2} \theta\left(r-r_{0}\right)\left(r-r_{0}\right) e^{-\gamma\left(r-r_{0}\right)}
$$

$\operatorname{com} \theta(x)$ é a função degrau, o parâmetro $r_{0}$ calculado a partir da polarizabilidade média da molécula utilizando a equação (5.5) e o parâmetro variacional $\gamma$ que deve minimizar o valor esperado do Hamiltoniano (em unidades atômicas):

$$
\langle\hat{H}\rangle_{\gamma}=-\int\left(r \phi_{d} \frac{1}{2} \frac{\partial^{2}}{\partial r^{2}}\left(r \phi_{d}\right)+\phi_{d} V \phi_{d} r^{2}\right) d r
$$




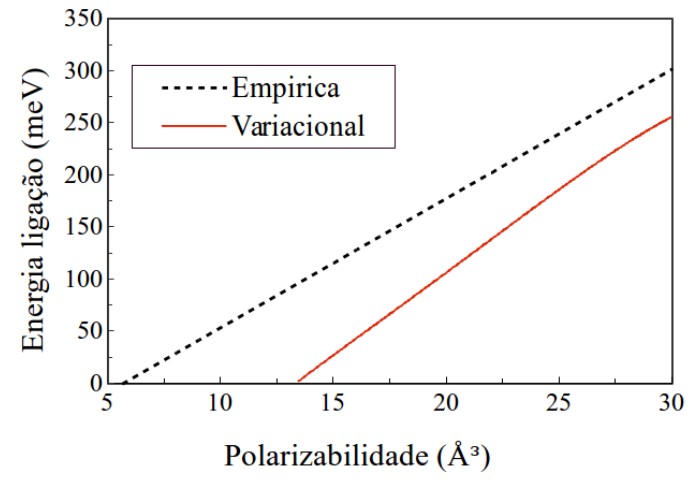

(A)

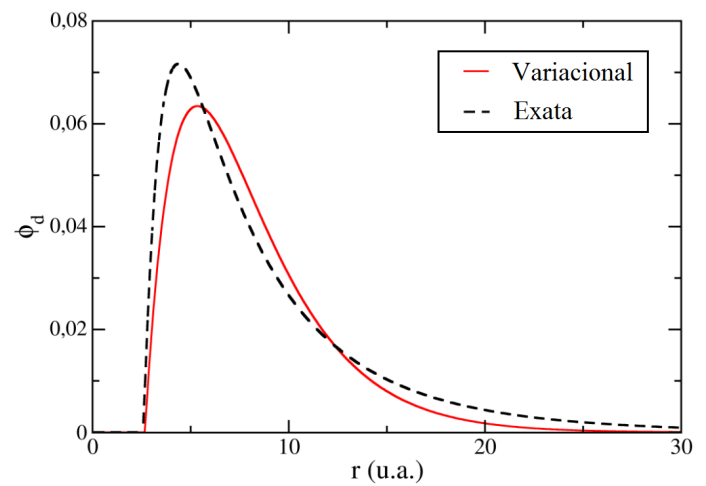

(B)

FigurA 5.2: (a) Comparação do valor absoluto da energia de ligação (cinza-sólida) prevista pela equação (5.3) e (preta-pontilhada) a obtida, com o método variacional equação (5.8), para diferentes valores de polarizabilidade. (b) Comparação da função de onda numérica (linha contínua) com respeito à função de onda variacional (linha tracejada) para $\alpha=15 \AA^{3}$.

o funcional é dependente de $\gamma$ e parametricamente da polarizabilidade $(\alpha)$. Ao substituir a função de onda $\phi_{d}$ dada por (5.6), obtemos:

$$
\langle\hat{H}\rangle=\frac{1}{2\left(3+3 t+t^{2}\right) r_{0}^{2}}\left[t^{2}\left(1+t+t^{2}\right)-\frac{4 \alpha}{r_{0}^{2}}\left(\frac{t^{4}}{2}-t^{5} e^{2 t}\left(2 E_{1}(2 t)-E_{2}(2 t)\right)\right)\right],
$$

onde é definida a variável adimensional $t=\gamma r_{0}$ e utilizada a função exponencial integral de ordem $n E_{n}(x)=\int_{1}^{\infty} u^{-n} e^{-x u} d u[49]$. O parâmetro $t$ foi modificado de 0 até $10 \mathrm{em}$ passos de 0.001 para determinar o mínimo do funcional $\langle\hat{H}\rangle$, esse valor mínimo pode ser interpretado como a energia de ligação $\varepsilon_{b}$ para essa função de onda.

Os valores de $\varepsilon_{b}$ obtidos para diferentes valores de $\alpha$ permitiram observar que nosso modelo só permite obter estados ligados para $\alpha>13.2 \AA^{3}$ enquanto que a equação (5.3) e a solução numericamente exata do hamiltoniano associado a (5.4), preveem estados ligados para $\alpha>5.6 \AA^{3}$. A comparação entre as duas energias de ligação: a prevista pelo modelo (eq (5.8) minimizada) e a fórmula empírica (5.3) como função da polarizabilidade, é mostrada na figura $5.2 \mathrm{a}$.

É importante notar, porém, que, embora exista uma diferença significativa entre energias de ligação do modelo numérico e o variacional, este último mantém a relação aproximadamente linear entre $\varepsilon_{b}$ e $\alpha$ da maneira prevista qualitativamente pela equação (5.3). A função de onda obtida pelo método variacional (5.6) não é muito diferente à solução de (5.4). Na figura 5.2b as duas funções são comparadas ${ }^{1}$.A principal diferença entre elas é

\footnotetext{
${ }^{1}$ Ambas soluções são normalizadas como $\int|\phi(r)|^{2} r^{2} d r=1$
} 
que a função (5.6) decai mais rápido com a distância do que a solução numérica. Pode-se observar tal qualidade na figura 5.2a.

O intervalo de energia no qual o modelo variacional funciona rigorosamente, segundo a figura 5.2a $\alpha \in(13.5,30.3) \AA^{3}$ é um limitante uma vez que a maior parte de moléculas estudadas têm polarizabilidades médias, menores que $10 \AA^{3}$. Para confrontar o problema, foi observada a dependência de $\gamma$ em relação à polarizabilidade média $(\alpha)$. Uma extrapolação da tendência, como é apresentado na figura (5.3a), permitiu construir funções de onda para polarizabilidades pequenas embora elas não descrevam, rigorosamente, soluções que representem um estado ligado. O parâmetro $\gamma$, dado um valor de $\alpha$, pode ser calculado utilizando a função:

$$
\gamma=0.4011^{-1}+\left(3338 \alpha-103.9 \alpha^{2}+1.125 \alpha^{3}\right) \times 10^{-5} A^{-1}
$$

Para averiguar a validade da extrapolação, foi realizada uma comparação entre as duas

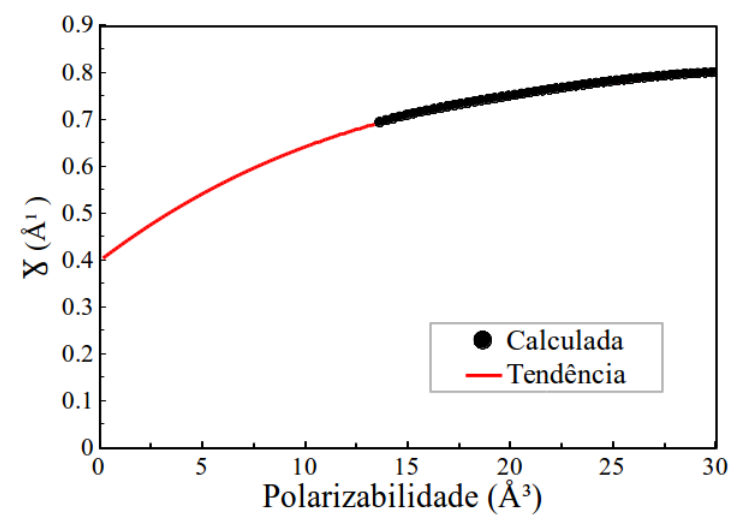

(A)

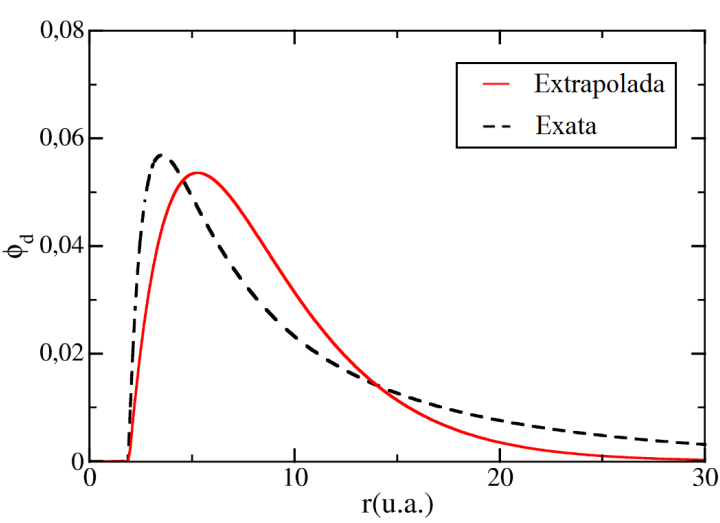

(B)

FigurA 5.3: (a) Extrapolação do parâmetro variacional $\gamma$ que minimiza (5.7) em função da polarizabilidade da molécula (b) Comparação da função de onda numérica (linha tracejada) com a função de onda obtida (linha continua) com o parâmetro $\gamma$ extrapolado a $\alpha=6.0 \AA^{3}$.

funções de onda: a analítica extrapolada e a numericamente exata obtidas para a polarizabilidade de $6 \AA^{3}$ sendo ambas apresentadas na figura 5.3b. A comparação, sugere que o erro cometido não é grande. Lembramos de novo, que o propósito do modelo não é ser rigoroso mas fazer uma estimativa da ordem de grandeza de $\Gamma_{\nu}^{e}$ e compará-la com algumas obtidas no formalismo de Gribakin-Lee para transições IR-ativas.

Uma das principais vantagens obtidas com a fórmula (5.9) é a possibilidade de obter soluções aproximadas para qualquer valor de polarizabilidade. O método será validado pela comparação das larguras obtidas $\Gamma_{\nu}^{e}$, com a função de onda extrapolada e a numericamente exata. A superfície de energia potencial não será modificada com o acoplamento 
do pósitron mas simplesmente por um deslocamento vertical de energia $\left(\varepsilon_{b}\right)$, da mesma forma aproximada no modelo GL. Assim, o acoplamento do pósitron levará a transições vibracionais simples $0 \rightarrow 1$.

Uma vez estimada a função de onda do estado ligado $\phi_{d}$, utilizando o potencial regularizado dado pela equação (5.4), para a estimativa da largura, foi utilizada a equação (C.5):

$$
\Gamma_{\nu}^{e}=8 \pi^{2} k_{\nu}\left|U_{k_{\nu}}\right|^{2} \quad ; \quad k_{\nu}=\sqrt{2\left(\omega_{\nu}+\varepsilon_{b}\right)}
$$

onde $\omega_{\nu}$ é a energia da transição vibracional, e $\varepsilon_{b}<0$, é a energia de ligação. ${ }^{2} \mathrm{~A}$ amplitude de transição $U_{k}$, definida na equação (4.11), pode ser escrita, ao substituir $V_{\text {cap }}$ pelo potencial (5.4), da maneira:

$$
\begin{aligned}
U_{\mathbf{k}} & =\left\langle\nu\left|\left\langle\phi_{d}\left|V_{\text {cap }}\right| \mathbf{k}\right\rangle\right| 0\right\rangle \\
U_{\mathbf{k}} & =-\langle\nu|\alpha| 0\rangle \int d \Omega_{\mathbf{r}} \int_{r_{0}}^{\infty} r^{2} d r \phi_{d}(r) \frac{1}{2 r^{4}} \frac{e^{i \mathbf{k} \cdot \mathbf{r}}}{(2 \pi)^{3}}
\end{aligned}
$$

onde é possível definir a amplitude de captura $A_{k}$ como a integral:

$$
A_{\mathbf{k}}=\int d \Omega_{\mathbf{r}} \int_{r_{0}}^{\infty} r^{2} d r \phi_{d}(r) \frac{1}{2 r^{4}} e^{i \mathbf{k} \cdot \mathbf{r}}
$$

Usando a função de onda $\phi_{d}(r)$ do tipo (5.6) que corresponde ao mínimo de (5.8) e integrando sobre as coordenadas do pósitron, a amplitude de transição do pósitron (5.12) pode ser escrita na forma:

$$
\begin{aligned}
A_{k}= & \sqrt{\frac{4 \pi\left(t / r_{0}\right)^{5}}{3+3 t+t^{2}}} \frac{1}{s}\left[\left(\frac{t}{2}+\frac{1}{2} \sin (s)-\frac{s}{2} \cos (s)\right)+\right. \\
& \left.e^{t}\left(\left(\frac{s^{2}}{2}-\frac{t^{2}}{2}-t\right) \operatorname{Im}\left(E_{1}(t-i s)\right)+s(1+t) \operatorname{Re}\left(E_{1}(t-i s)\right)\right)\right]
\end{aligned}
$$

onde os parâmetros adimensionais $t=\gamma r_{0}$ e $s=k r_{0}$ dependem do raio de corte $\left(r_{0}\right)$ e do parâmetro variacional $(\gamma)$, calculados a partir da polarizabilidade média $(\alpha)$ usando as equações (5.5) e (5.9), respectivamente, além do número de onda do pósitron no contínuo $(k)$. A função $E_{n}(a+i b)=\int_{1}^{\infty} u^{-n} e^{-(a+i b) u} d u$ é chamada Função Gamma incompleta de argumento complexo[49].

Com o propósito de avaliar a função de onda variacional em relação à função de onda obtida numéricamente do Hamiltoniano associado ao potencial (5.4), foram realizados cálculos da probabilidade de captura $\left(\left|A_{k}\right|^{2}\right)$ em função da energia $\left(k^{2} / 2\right)$ do estado do pósitron incidente, utilizando as duas funções de onda, a variacional e a numericamente

\footnotetext{
${ }^{2} \mathrm{O}$ termo $8 / p i^{2}$ pode mudar dependendo da definição da nomalização da função de onda
} 


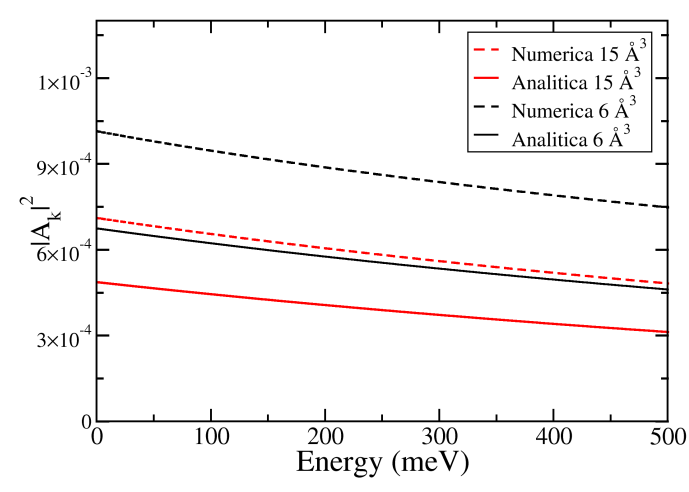

(A)

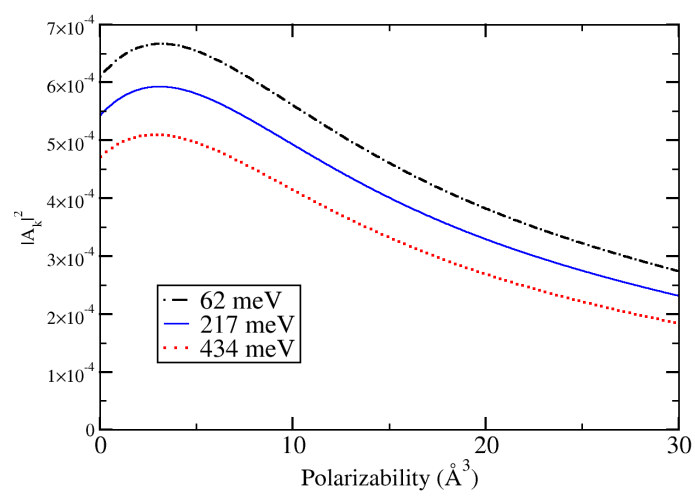

(B)

Figura 5.4: (a) Módulo ao quadrado da amplitude de captura, $\left|A_{k}\right|^{2}$, calculado com as duas funções: a solução numericamente exata (linha tracejada) e a aproximação variacional (linha continua) em função da energia de transição do acoplamento calculadas, para valores de $\alpha=15 \AA^{3}$ e $\alpha=6 \AA^{3}$. (b) $\left|A_{k}\right|^{2}$ em função da polarizabilidade $(\alpha)$ para alguns valores típicos de energia de transições vibracionais.

exata. A comparação de $\left|A_{k}\right|^{2}$ num intervalo de energia de 0-500 $\mathrm{meV}^{3}$ para dois valores de polarizabilidade ( $\alpha=6 \AA^{3}$ e $\alpha=15 \AA^{3}$ ) é apresentada na figura (5.4a).

É possível dar conta que a função de onda exata produz um $\left|A_{k}\right|^{2}$ qualitativamente similar ao obtido com os cálculos da função de onda analítica mas ligeiramente maior por uma quantidade aproximadamente constante $\left(\approx 2 \times 10^{-4}\right.$ para $\alpha=15 \AA^{3}, \approx 3 \times 10^{-4}$ para $\left.\alpha=6 \AA^{3}\right)$. Tais diferenças, no entanto, não são relativamente grandes e o essencial, do ponto de vista do nosso interesse, é que as estimativas têm a mesma ordem de grandeza. Dessa maneira, para nós, a aproximação variacional é tão razoável quanto a solução numérica.

Os cálculos realizados usando as duas soluções preveem, ao aumentar a energia da transição do acoplamento, um decrescimento de $\left|A_{k}\right|^{2}$ suave. Especificamente, ao aumentar a energia duas ordens de grandeza (de 1 até $500 \mathrm{meV}$ ), o decrescimento de $\left|A_{k}\right|^{2}$ é apenas $\sim 15 \%$ para ambos modelos e, portanto, usar o modelo variacional não levaria a erros importantes.

Uma vez que o modelo analítico permite o cálculo de $\left|A_{k}\right|^{2}$ sem precisar resolver exatamente o problema para cada valor de $\alpha$, foram feitos cálculos de $\left(\left|A_{k}\right|^{2}\right)$ em função da polarizabilidade $(\alpha)$ e apresentados na figura (5.4b). Foram feitos cálculos para alguns valores de energia de transição vibracional típicos ou de energia incidente do pósitron uma vez que $k^{2} / 2=\omega_{\nu}+\varepsilon_{b}$. Na figura, é possível notar que, $\left(\left|A_{k}\right|^{2}\right)$ não depende fortemente da polarizabilidade $(\alpha)$ pois sua variação para um amplo intervalo também é

\footnotetext{
${ }^{3}$ intervalo de energia típico das transições vibracionais
} 
relativamente pequena. Portanto, segundo a equação (5.10), o acoplamento vibracional $\left|\alpha_{\nu}\right|^{2}$ será a fonte principal de variações no valor da largura $\left(\left|U_{k}\right|^{2}\right)$.

\subsection{Cálculo de $\Gamma_{\nu}^{e}$}

Com a ideia de comparar as larguras para transições ativas no infravermelho e para transições Raman-ativas, foram calculadas as larguras para o espectro completo das moléculas de Acetileno $\left(\mathrm{C}_{2} \mathrm{H}_{2}, \alpha=3.3 \AA^{3}\right.$ e $\left.\varepsilon_{0} \approx-5 \mathrm{meV}\right)$, Etileno $\left(\mathrm{C}_{2} \mathrm{H}_{4}, \alpha=4.2\right.$ $\AA^{3}$ e $\left.\varepsilon_{0} \approx-10 m e V\right)$ e Etano $\left(\mathrm{C}_{2} \mathrm{H}_{6}, \alpha=4.4 \AA^{3}\right.$ e $\left.\varepsilon_{0} \approx-5 m e V\right)[43]$ uma vez que os seus espectros vibracionais incluem tanto transições IR-ativas, quanto transições Ramanativas. A comparação de larguras é apresentada na tabela 5.2.

\begin{tabular}{lccr}
\multicolumn{4}{c}{ Etano $\left(\mathrm{C}_{2} \mathrm{H}_{6}\right)$} \\
\cline { 1 - 4 } Modo & Simetria & $\omega_{\nu}(\mathrm{meV})$ & $\Gamma_{\nu}^{e}(\mathrm{meV})$ \\
\hline 1 & $A_{g}$ & 376 & 0.146 \\
2 & $A_{g}$ & 174 & 0.515 \\
3 & $A_{g}$ & 125 & 0.155 \\
4 & $A_{u}$ & 38 & 3.63 \\
5 & $A_{2 u}$ & 376 & 0.468 \\
6 & $A_{2 u}$ & 171 & $6.38 \times 10^{-3}$ \\
7 & $E_{g}$ & 383 & 0.256 \\
8 & $E_{g}$ & 182 & - \\
9 & $E_{g}$ & 149 & 0.5525 \\
10 & $E_{g}$ & 385 & 0.518 \\
11 & $E_{g}$ & 183 & 0.0645 \\
12 & $E_{g}$ & 101 & 0.0252 \\
\hline
\end{tabular}

TABELA 5.1: Larguras de ressonância para as transições vibracionais calculadas com o modelo GL para modos no infravermelho (Branco) com o modelo para modos Ramanativos (cinza) para a molécula de Etano $\left(\mathrm{C}_{2} \mathrm{H}_{6}, \alpha=4.4 \AA, \varepsilon \approx 5 \mathrm{meV}\right)$. A partir dos cálculos de estrutura eletrônica realizados no nível DFT/B3LYP/aug-cc-pVDZ.

Aqui, as larguras $\left(\Gamma_{\nu}^{e}\right)$ para transições Raman-ativas, são calculadas utilizando a expressão analítica para $A_{k}$ dada pela equação (5.13) utilizando os parâmetros $r_{0}$ e $\gamma$ dados pelas equações (5.5) e (5.9) calculados a partir da polarizabilidade da molécula $(\alpha)$. Os modos que não têm valor são aqueles não ativos para excitação nem uma.

Uma vez que a função de onda empregada nos cálculos (5.6) não é rigorosamente um estado ligado, é utilizada a energia de ligação $\varepsilon_{b}$ medida no experimento para construir o argumento $k_{0 \nu}$ definido na equação (5.10) utilizando também a frequência de transição $\omega_{\nu}$ obtida a partir de cálculos de estrutura eletrônica no nível DFT/B3LYP/aug-cc-pVDZ. Esses mesmos cálculos foram utilizados para obter a amplitude de transição vibracional $\alpha_{\nu}$ utilizando a equação (3.27) a partir dos valores de atividade Raman $\left(S_{i}\right)$ e depolarização $\left(\rho_{i}\right)$. Para acoplamentos com transições ativas no infravermelho, as $\Gamma_{\nu}^{e}$ foram 


\begin{tabular}{lccr}
\multicolumn{4}{c}{ Etileno $\left(\mathrm{C}_{2} \mathrm{H}_{4}\right)$} \\
\hline Modo & Simetria & $\omega_{\nu}(\mathrm{meV})$ & $\Gamma_{\nu}^{e}(\mathrm{meV})$ \\
\hline 1 & $A_{g}$ & 390 & 0.0251 \\
2 & $A_{g}$ & 208 & 0.398 \\
3 & $A_{g}$ & 169 & 0.699 \\
4 & $A_{u}$ & 129 & - \\
5 & $B_{1 u}$ & 388 & 0.124 \\
6 & $B_{1 u}$ & 180 & 0.0740 \\
7 & $B_{2 g}$ & 122 & - \\
8 & $B_{2 u}$ & 401 & 0.168 \\
9 & $B_{2 u}$ & 102 & - \\
10 & $B_{3 g}$ & 398 & - \\
11 & $B_{3 g}$ & 152 & - \\
12 & $B_{3 u}$ & 122 & 0.762 \\
\hline
\end{tabular}

\begin{tabular}{lccr}
\multicolumn{4}{c}{ Acetileno $\left(\mathrm{C}_{2} \mathrm{H}_{2}\right)$} \\
\hline Modo & Simetria & $\omega_{\nu}(\mathrm{meV})$ & $\Gamma_{\nu}^{e}(\mathrm{meV})$ \\
\hline 1 & $\Sigma_{g}$ & 376 & 0.102 \\
2 & $\Sigma_{g}$ & 174 & 0.555 \\
3 & $\Sigma_{u}$ & 125 & 0.729 \\
4 & $\Pi_{g}$ & 38 & - \\
5 & $\Pi_{u}$ & 376 & 0.770 \\
\hline
\end{tabular}

TABELA 5.2: Larguras de ressonância para as transições vibracionais calculadas com o modelo GL para modos no infravermelho (Branco) com o modelo para modos Ramanativos (cinza) para as moléculas de Etileno $\left(\mathrm{C}_{2} \mathrm{H}_{4}, \alpha=4.2 \AA, \varepsilon \approx 10 \mathrm{meV}\right)$ e o Acetileno $\left(\mathrm{C}_{2} \mathrm{H}_{2}, \alpha=3.3 \AA, \varepsilon \approx 5 \mathrm{meV}\right)$. A partir dos cálculos de estrutura eletrônica realizados no nível DFT/B3LYP/aug-cc-pVDZ.

estimadas utilizando a equação (3.15) a partir das intensidades infravermelhas definidas na equação (3.11) obtidas pelos mesmos cálculos de estrutura eletrônica utilizados para os modos Raman-ativos.

Nas tabelas 5.1 e 5.2, é possível observar que a ordem de grandeza de $\Gamma_{\nu}^{e}$ é semelhante para os dois tipos de transições vibracionais, confirmando a relação (5.1) permitindo concluir que os tempos de vida das ressonâncias são muito similares e independentes do tipo de transição vibracional. Dentro das qualidades do método baseado na função de onda variacional, mencionamos que da mesma forma que o método de Gribakin-Lee, há somente um parâmetro livre, a energia de ligação $\left(\varepsilon_{b}\right)$.

Como foi sugerido depois do análise das figuras $5.4 \mathrm{a}$ e $5.4 \mathrm{~b}$, possivelmente $\left|A_{k}\right|^{2}$ poderia ser tratado como constante para qualquer transição vibracional. Para verificar essa hipótese, foram calculados, da maneira exposta anteriormente, além das apresentadas na tabela 5.1a, as larguras para as transições Raman-Ativas do Butano $\left(\mathrm{CH}_{3} \mathrm{CH}_{2} \mathrm{CH}_{2} \mathrm{CH}_{3}\right.$, $\left.\alpha=8.1 \AA^{3}, \varepsilon_{b} \approx 35 \mathrm{meV}\right)[43]$.

Assim, tendo em conta que $\Gamma_{\nu}^{e}$ é dada, explicitamente por 


$$
\Gamma_{\nu}^{e}=\frac{1}{\pi} \sqrt{2\left(\omega_{\nu}+\varepsilon_{b}\right)}\left|\alpha_{\nu}\right|^{2}\left|A_{\nu}\right|^{2}=\frac{1}{\pi} k_{\nu}\left|\alpha_{\nu}\right|^{2}\left|A_{\nu}\right|^{2}
$$

se $\left|A_{k}\right|^{2}$ pode ser aproximada por uma constante, um gráfico que relacione as larguras $\Gamma_{\nu}^{e}$, com seu respetivo $k_{\nu}\left|\alpha_{\nu}\right|^{2}$, será, aproximadamente uma linha reta e sua inclinação será $m=\left|A_{\nu}\right|^{2} / \pi$. Esse gráfico é a presentado na figura 5.5:

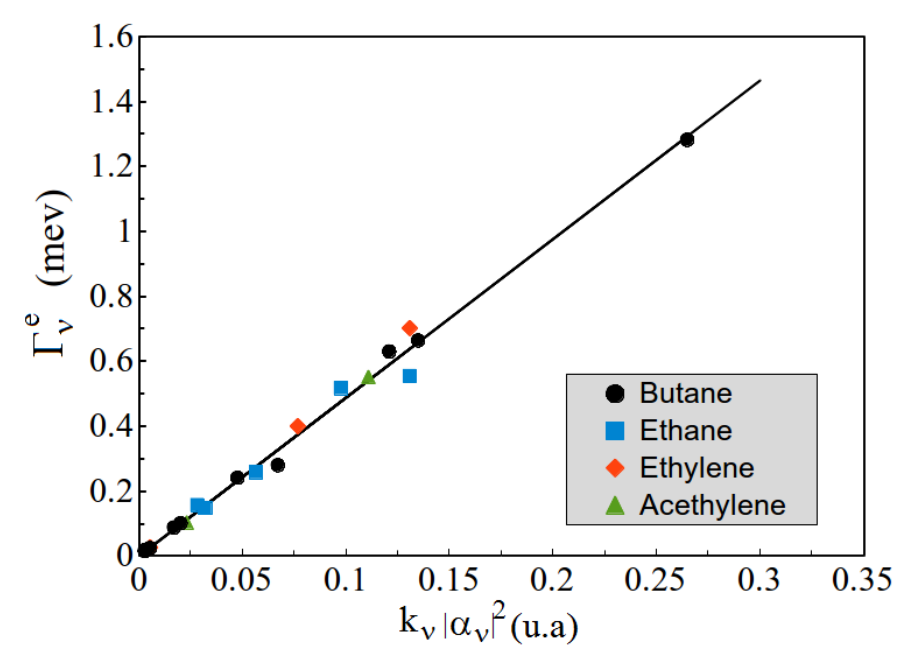

Figura 5.5: $\Gamma_{\nu}^{e}(\mathrm{meV})$ para algumas transições Raman-ativas em função de $k_{\nu}\left|\alpha_{\nu}\right|^{2}$ para quatro moléculas.

Da figura 5.5, pode-se obter a simples relação:

$$
\Gamma_{\nu}^{e}(\mathrm{meV})=4.88 \times k_{\nu}\left|\alpha_{\nu}\right|^{2}(\text { u.a. })
$$

A fórmula 5.15 resume todas as características encontradas no cálculo de $\Gamma_{\nu}^{e}$ para modos Raman-ativos e pode ser usada, de maneira simples, a partir da amplitude de transição $\left(\left|\alpha_{\nu}\right|\right)$, dada pela equação (3.27). Cabe destacar que $\alpha_{\nu}$ é uma característica da molécula isolada, de maneira que conhecendo o espectro vibracional da molécula isolada, é possível fazer uma estimativa de primeira ordem de $Z_{\text {eff }}$, no entanto, sem uma maneira de calcular o deslocamento $\varepsilon_{b}$ dos picos a partir de primeiros princípios. O fator 4.88 da equação (5.15) dá uma estimativa, em primeira ordem, do acoplamento eletrônico do pósitron, no entanto, não tem em conta efeitos importantes que serão estudados na seguinte seção.

Finalmente, seria oportuno observar que $\Gamma_{\nu}^{e}$ pode envolver um intervalo de energia relativamente grande, desde $10^{-2} \mathrm{meV}$ até $10 \mathrm{meVs}$. Tal propriedade poderá incidir no $Z_{\text {eff }}$ se a molécula apresentasse algum mecanismo de redistribuição de energia vibracional.[23] Se $\Gamma_{\nu}^{e}$ para certa transição $\nu$ é relativamente grande, esse modo vai ser usado para ejetar o pósitron e se a energia dos outros modos, por mecanismos de redistribuição, consegue chegar até ele, $Z_{\text {eff }}$ para essa transição específica será menor ao esperado uma vez que o 
pósitron terá mais probabilidade de ser ejetado. E se a energia da vibração é levada a

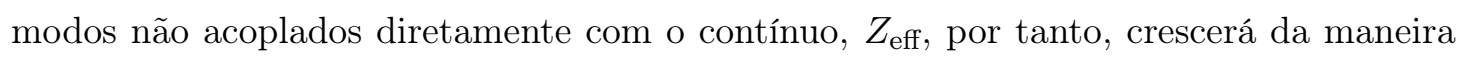
como será abordado no capítulo seguinte.

\subsection{Conclusões}

Foi apresentado um método analítico para o cálculo de larguras de acoplamento do pósitron em transições Raman-ativas mediado pelo potencial de acoplamento regularizado dado pela equação (5.4). O modelo funciona para moléculas no intervalo de polarizabilidade $(13.2,30) \AA^{3}$ que, no entanto, foi extrapolado de maneira que foi utilizado no intervalo $(0,30) \AA^{3}$ com a energia de ligação $\left(\varepsilon_{b}\right)$ como parâmetro livre.

Foi observado que a probabilidade de captura do pósitron $\left(A_{\nu}\right)$ é aproximadamente constante no intervalo de polarizabilidade e energia do pósitron incidente, de maneira que a maior parte de variação de $\Gamma_{\nu}^{e}$ é dada pela amplitude de transição vibracional que foi aproximada à da molécula isolada $\alpha_{\nu}$. As propriedades do modelo são resumidas, finalmente, pela relação:

$$
\Gamma_{\nu}^{e}(\mathrm{meV})=4.88 \times k_{\nu}\left|\alpha_{\nu}\right|^{2}(\text { u.a. })
$$

onde observamos que, segundo a estimativa, a ordem de grandeza das larguras para modos Raman-ativos e para modos IR-ativos é semelhante variando desde centésimos de meV até alguns poucos meV mantendo a relação (5.1). 


\section{Capítulo 6}

\section{Acoplamentos vibracionais mediados pela correlação elétron-pósitron.}

\subsection{Introdução}

Nas aproximações feitas atualmente para modelar o acoplamento pósitron molécula, são desprezados os efeitos de correlação elétron-pósitron. Usualmente, são usados parâmetros da molécula isolada como seu momento de dipolo $(\boldsymbol{\mu})$, sua polarizabilidade média $(\alpha)$ e seu espectro vibracional de maneira que efeitos próprios da correlação elétron-pósitron não são levados em conta. As teorias para modelar o acoplamento do pósitron, são admitidos efeitos de polarização mas não da correlação elétron-pósitron.

Em publicações anteriores de nosso grupo de pesquisa[23], foi abordado o problema de outro ponto de vista. Desprezando as transições vibracionais devidas explicitamente ao acoplamento contínuo-discreto de pósitron, foram abordados possíveis acoplamentos vibracionais como consequência das deformações da superfície de energia potencial (SEP) da molécula induzidas pela correlação do pósitron e a nuvem eletrônica[23]. Tais deformações são tratadas perturbativamente a partir da SEP da molécula isolada na sua base de auto-estados vibracionais. Uma vantagem de esse tipo de acoplamento é a possibilidade de acoplar com o contínuo, não somente os canais de entrada, mas modos de vibração múltiplos e overtones. Fato que é consistente com os experimento[42]. No entanto, o cálculo ad hoc de perturbações da SEP do composto pósitron-molécula precisa de técnicas computacionais que atualmente são um ramo ativo de pesquisa.

Neste capítulo, serão revisitadas as transições vibracionais induzidas pela captura do pósitron mas incluindo acoplamentos vibracionais adicionais ao considerar a correlação elétron-pósitron na superfície de energia potencial fazendo uma estimativa, a partir de 
cálculos de estrutura eletrônica, da forma na que a perturbação pode acoplar níveis excitados da molécula e fazendo uma discussão do papel desses efeitos na excitação de overtones discutindo o acoplamento seletivo, a supressão e o aprimoramento de picos em $Z_{\text {eff. }}$

\subsection{Métodos computacionais para o cálculo de estrutura eletrônica com pósitrons}

Para fazer uma estimativa dos efeitos de correlação elétron-pósitron, será resolvido o hamiltoniano eletrônico para a molécula isolada e para o composto pósitron-molécula. Uma boa parte dos métodos computacionais utilizados para o cálculo de estrutura eletrônica de compostos que incluem o pósitron é uma generalização dos métodos computacionais utilizados nos cálculos de estrutura eletrônica usuais[50]. Uma visão geral das principais técnicas utilizadas nos cálculos de estrutura eletrônica e que vão ser utilizadas no capítulo, é apresentada no apêndice A.

O método utilizado aqui para lidar com o cálculo de estrutura eletrônica do composto é o método de Orbital Nuclear-Eletrônico (NEO pelas suas iniciais em inglês),[51]. Ele foi originalmente desenvolvido para incluir efeitos quânticos em núcleos ao mesmo nível dos elétrons, ou seja, tratando os núcleos não como partículas pontuais mas descritas por uma função de onda[52] de maneira que a função de onda total é o produto de um determinante de Slater nuclear e outro determinante eletrônico:

$$
\Psi_{H F}\left(\mathbf{r}^{e}, \mathbf{r}^{p}\right)=\Phi^{e}\left(\mathbf{r}^{e}\right) \Psi^{p}\left(\mathbf{p}^{p}\right)
$$

aqui, $\mathbf{r}^{e}$ representa as coordenadas eletrônicas, $\mathbf{r}^{p}$ representa as coordenadas do próton quântico ou do pósitron. Ao nível Hartree-Fock-NEO (NEO-HF), portanto, as funções de onda positrônica e eletrônica são calculadas de maneira iterativa usando técnicas variacionais de orbital molecular. No caso dos pósitrons, eles são tratados como um núcleo quântico de Hidrogênio mas com a massa do pósitron. As correlações elétronelétron e elétron-pósitron podem ser incluídas por teoria de perturbação Moller-Plesset de segunda ordem (NEO-MP2), a aproximação de interação de configuração completa (NEO-FCI) e o campo auto-consistente de espaço ativo completo (NEO-CASSCF).[52]

Embora o método seja útil ao levar em conta os efeitos quânticos desejados, não é a melhor ferramenta para assegurar uma boa precisão. Atualmente, cálculos bem convergidos por métodos variacionais estocásticos, implementados principalmente pelo grupo de Tachikawa[42], conseguem uma energia para o PsH de -0.789196740 Hartree e uma taxa de aniquilação de $2.47178 n s^{-1}[53]$, sendo utilizada uma base de 1800 gaussianas 
explicitamente correlacionadas enquanto, o método NEO-MP2 para uma base de pósitron [6s3p1d] consegue uma energia de -0.728306 Hartree e uma taxa de aniquilação de $0.537651 \mathrm{~ns}^{-1}[54]$.

\subsection{Cálculos de curvas de energia potencial para moléculas diatômicas}

Este capitulo está centrado na ideia de que a deformação da superfície de energia potencial (SEP) decorrente da captura do pósitron, permitirá acoplar estados vibracionais da molécula isolada definindo os estados vibracionais do composto. Até agora, a maneira como as deformações da SEP eram consideradas, utilizava parâmetros empíricos ao invés de estimativas por primeiros princípios[23]. Graças ao trabalho conjunto desde o segundo semestre de 2013 com o grupo de pesquisa de química quântica da Universidade Nacional de Colômbia, liderado pelo professor Andrés Reyes, com experiência no cálculo de estrutura eletrônica para moléculas com sistemas de mais de uma espécie quântica, foram feitos alguns cálculos de superfícies de energia potencial para sistemas com pósitrons.

Os primeiros sistemas escolhidos para as estimativas da correlação elétron-pósitron, neste trabalho, foram moléculas diatômicas pois podem ser descritas, simplesmente, por um modo de vibração. Especificamente, foram escolhidos os hidretos de metais alcalinos $\mathrm{LiH}$ e $\mathrm{NaH}$ uma vez que, além de ser diatômicas, correspondem a moléculas com um momento dipolar grande de maneira que produzirão compostos pósitron-molécula mais estáveis permitindo ilustrar de uma melhor maneira os efeitos de correlação.

A deformação das curvas de potencial (CEP) para este tipo de hidretos, desde LiH até $\mathrm{RbH}$, incluindo $\mathrm{NaH}$ e KH, já foi reportada no ano 2006 pelo grupo de Tachikawa[56]. $\mathrm{Na}$ aproximação de Born-Oppenheimer, foram obtidas com o método interação de configurações multi-referencial com excitações simples e duplas (MRD-CI). As curvas de energia potencial, para as moléculas de $\mathrm{LiH}$ e $\mathrm{NaH}$, isoladas e para seus correspondentes compostos pósitron-molécula são apresentadas nas figuras 6.1a. e 6.2a

Para o trabalho, foi disposto do nível MP2 de cálculo (ver apêndice A.3.1). De maneira que para comparar a acurácia desse nível no cálculo da correlação elétron-pósitron, em relação ao nível MRD-CI, foram levantadas as curvas de energia potencial (CEP) para a molécula diatômica $\mathrm{LiH}$ e o composto $e^{+} \mathrm{LiH}$ sendo elas apresentadas na figura 6.1b. Da mesma maneira são apresentados os mesmos cálculos da CEP para a molécula NaH e o composto $e^{+} \mathrm{NaH}$ na figura $6.2 \mathrm{~b}$ 


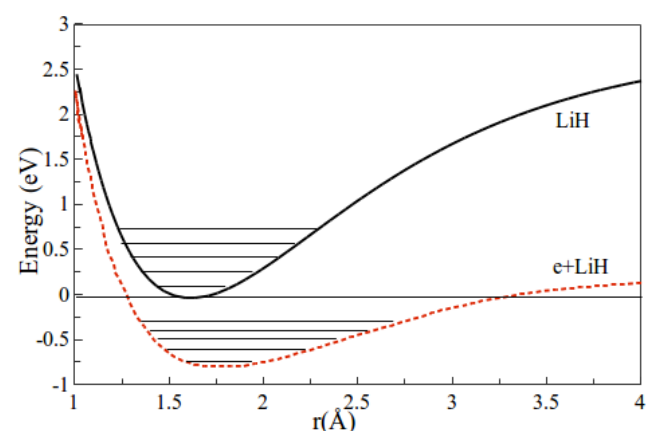

(A)

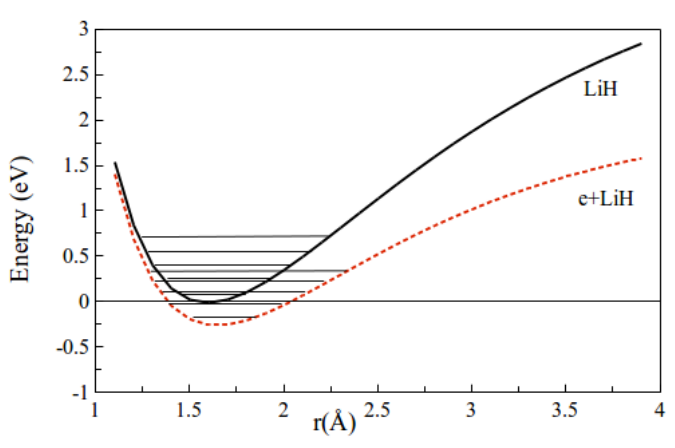

(B)

Figura 6.1: Curvas de energia potencial para a molécula de $\mathrm{LiH}$ e $e^{+} \mathrm{LiH}$ calculadas (A) no nível MRD-CI obtidas da referência[56] (B) no nível UHF-NEO-MP2 para bases eletrônicas 6-311++G(df, pd) e positrônica 10s10d10p. Para cada curva, são mostrados os respectivos níveis vibracionais.

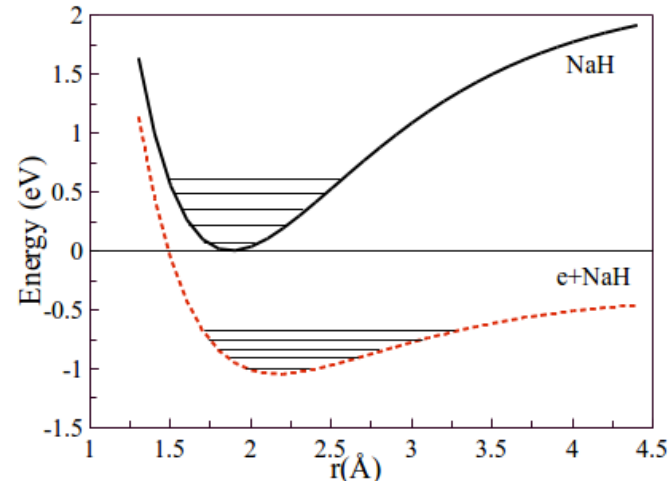

(A)

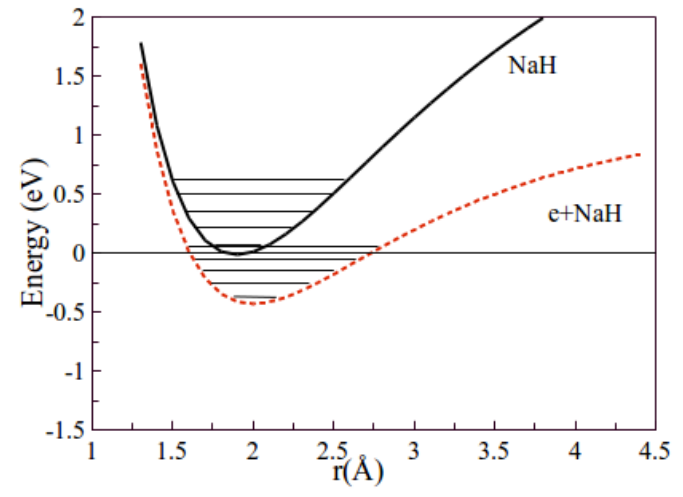

(B)

Figura 6.2: Curvas de energia potencial para a molécula de $\mathrm{NaH}$ e $e^{+} \mathrm{NaH}$ calculadas (A) no nível MRD-CI obtidas da referência[56] (B) no nível UHF-NEO-MP2 para bases eletrônicas 6-311 $++\mathrm{G}(\mathrm{df}$, pd) e positrônica 10s10d10p. Para cada curva, são mostrados os respectivos níveis vibracionais.

Uma vez que o propósito dos cálculos computacionais da estrutura eletrônica da molécula é dar um entendimento quantitativo dos acoplamento vibracionais resultantes da deformação da CEP, os dados das CEP foram ajustados pelo método de mínimos quadrados, à equação de um potencial de Morse que é brevemente introduzido no apêndice B.2.

$$
V(r)=D_{0}\left(1-e^{-a\left(r-r_{0}\right)}\right)^{2}+V_{0}
$$

com $V_{0}$ a energia do mínimo, $r_{0}$ o comprimento de equilíbrio do sistema, ambos mostrados na tabela 6.1. $D_{0}$ e a parâmetros geométricos do potencial os quais, a partir da sua obtenção pelo ajuste dos dados, fazem possível calcular os níveis de energia $(\nu)$ da equação 
de Schrödinger. Eles satisfazem:

$$
E(\nu)=\omega_{0}(\nu+1 / 2)-\frac{\left[\omega_{0}(\nu+1 / 2)\right]^{2}}{4 D_{0}}+V_{0}
$$

com $\omega_{0}=a \sqrt{2 D_{0} / m}$, frequência apresentada na tabela 6.1. Estes níveis, $E(\nu)$ diferem dos níveis do oscilador harmônico simples pelo termo adicional de anarmonicidade $\chi=$ $\omega_{0}^{2} / 4 D_{0}$ que é mostrado na tabela 6.1. Incluído o termo anarmônico, a frequência de transição vibracional desde o estado fundamental até o primeiro estado excitado é dada por

$$
\omega_{0 \rightarrow 1}=\omega_{0}-\omega_{0}^{2} / 4 D_{0}
$$

\begin{tabular}{lcccc} 
Composto & $\mathrm{V}_{0}(\mathrm{eV})$ & $r_{0}(\AA)$ & $\omega_{0}(\mathrm{eV})$ & $\chi(\mathrm{meV})$ \\
\hline LiH (UHF-MP2) & 0.00 & 1.60 & 0.177 & 4.33 \\
LiH (MRD-CI) & 0.00 & 1.60 & 0.180 & 5.72 \\
e+LiH (UHF-MP2) & -0.263 & 1.65 & 0.161 & 5.93 \\
e+LiH (MRD-CI) & -0.796 & 1.76 & 0.133 & 8.20 \\
\hline NaH (UHF-MP2) & 0.00 & 1.92 & 0.148 & 3.63 \\
NaH (MRD-CI) & 0.00 & 1.87 & 0.150 & 5.12 \\
e+NaH (UHF-MP2) & -0.435 & 2.00 & 0.125 & 5.32 \\
e+NaH (MRD-CI) & -1.036 & 2.16 & 0.091 & 6.19 \\
\hline
\end{tabular}

TABEla 6.1: Energia da geometria de equilíbrio $\left(\mathrm{E}_{0}\right)$, separação de equilíbrio $\left(R_{0}\right)$ e frequências fundamentais $\left(\omega_{0}\right)$ e correção anarmônica $(\chi)$ para o $\mathrm{LiH}$ e e+ $\mathrm{LiH}$ e $\mathrm{NaH}$ e e+NaH ao nível de cálculo UHF-MP2 e MRD-CI

Para cada nível de cálculo apresentado na tabela $6.1, V_{0}$ é escolhido de forma que a energia do mínimo da curva da molécula isolada, seja zero. É possível observar que a variação do comprimento $\left(r_{0}\right)$ com respeito à geometria de equilíbrio no nível MRD-CI é cerca de 3 vezes o estiramento previsto no nível NEO-MP2 (3.2\% no nível NEO-MP2 contra $10 \%$ no nível MRD-CI). A variação da energia de equilíbrio $\left(E_{0}\right)$ novamente é cerca de 3 vezes maior no nível MRD-CI do que a obtida no nível NEO-MP2. Pode ser vista uma diminuição da concavidade da curva ao incluir o pósitron, de maneira que os níveis vibracionais têm uma separação menor, a correção de $\omega_{0}$ no nível MRD-CI é 3.4 vezes a correção no nível MP2.

No caso da molécula de NaH, vemos que as correções no nível MRD-CI são maiores do que para a molécula de LiH e comparadas com os cálculos no nível MP2, a correção na diferença de energia entre mínimos é 2.4 vezes maior, a correção no estiramento da molécula depois do acoplamento do pósitron é 3.6 vezes maior e a correção em $\omega_{0}$ é 3.9 vezes maior. 


\subsection{Processo de Acoplamentos vibracionais}

Rigorosamente, o operador $T_{N}+V_{\text {opt }}$ é um operador não hermítico uma vez que tem uma parte imaginaria $i \Gamma$ que como resultado não preserva a densidade de probabilidade ao evoluir no tempo. No entanto observando o sistema como un todo, essa densidade de probabilidade, que descreve o estad deslocalizado, está sendo transmitida a o estado deslocalizado de maneira que a densidade de probabbilidade total permanece constante.

Uma ves tomamos $\Gamma=\sum_{\nu} \Gamma_{\nu}$ uma soma de operadores constantes que agem em cada modo normal $\nu$ vemos que o operador $E-T_{N}-V_{\text {opt }}$ será diagonal na base de auto estados de $E-T_{N}-\operatorname{Re}\left(V_{\text {opt }}\right)$, o propósito de esta seção é apresentar como, conhecendo esses autoestados, é constroido um método para obter um $Z_{\text {eff }}$ que leve em conta os efeitos de correlação pósitron-molécula.

\subsubsection{Correlação pósitron-elétron como um potencial}

Na representação das coordenadas da molécula isolada $\{\mathbf{R}\}$, o operador variação da SEP $\left(\varepsilon_{d}(\mathbf{R})\right)$ definido, pela equação (4.10), como a diferença entre as SEPs da molécula isolada $\left(V_{0}(\mathbf{R})\right)$ e a do composto $V_{\mu}(\mathbf{R})$, definida incluindo a correlação pósitron-elétron $\left(\left\langle\phi_{\mathrm{d}}\left|H_{\text {ele }}\right| \phi_{\mathrm{d}}\right\rangle\right)$ mais o level shift $\Delta(\mathbf{R})$ associado à ressonância segundo a equação:

$$
\bar{\varepsilon}_{d}(\mathbf{R})=\left\langle\phi_{\mathrm{d}}\left|H_{\text {ele }}\right| \phi_{\mathrm{d}}\right\rangle+\Delta(\mathbf{R})-V_{0}(\mathbf{R})=V_{\mu}(\mathbf{R})-V_{0}(\mathbf{R})
$$

Aqui, o operador $\Delta$ na representação de coordenadas, em geral, é um operador não local uma vez que depende explicitamente do operador energia cinética dos núcleos. Usualmente é desprezado o cálculo explícito de $\Delta[23,39]$ no entanto, já têm sido feitas algumas estimativas dele para moléculas pequenas[55], não vai ser calculado neste trabalho mas é preciso lembrar que tanto a correlação elétron-pósitron, quanto $\Delta$, definem a dinâmica do composto.

As ferramentas computacionais de estrutura eletrônica disponíveis permitem fazer estimativas razoáveis da correlação elétron-pósitron permitindo fazer uma boa estimativa de $V_{\mu}(\mathbf{R})$ modelando-o por operadores cujo Hamiltoniano associado é resolúvel. Assim, trabalhar na base de estados vibracionais da molécula isolada $\left\{\eta_{\nu}\right\}$ quanto na base de estados vibracionais do composto pósitron-molécula $\left\{\mu_{\nu}\right\}$ é possível.

Por exemplo, na aproximação de GL, $V_{\mu}(\mathbf{R})=V_{0}(\mathbf{R})+\varepsilon_{b}$ onde $\varepsilon_{b}<0$ é uma constante e portanto os hamiltonianos associados a $V_{\mu}$ e $V_{0}$ compartilham o mesmo conjunto de autoestados mas com níveis de energia deslocados $\varepsilon_{b}$. Como pode ser visto nas figuras $6.2 \mathrm{a}$ 
e 6.1a, no caso geral, a diferença entre $V_{\mu}(\mathbf{R})$ e $V_{0}(\mathbf{R})$ não é tão simples, e portanto, a aproximação de Gribakin-Lee torna-se inadequada.

\subsubsection{Mecanismo de transição}

O operador $U_{\mathbf{k}}$ definido na equação (4.11) induz, simultaneamente a acoplamentos continuodiscreto do pósitron $\left(\left|\phi_{\mathbf{k}}\right\rangle\right.$ e $\left.\left|\phi_{d}\right\rangle\right)$ e transições entre os auto-estados vibracionais da molécula $\left(\left|\eta_{\nu}\right\rangle\right)$. Supondo que os auto-estados vibracionais do composto $\left\{\mu_{\nu}\right\}$ formam um conjunto completo, a partir de 4.17 , o pacote de onda do transiente $(|\xi\rangle)$ pode ser escrito da maneira:

$$
|\xi\rangle=\sum_{\mu}\left(E-T_{N}-V_{\mathrm{opt}}\right)^{-1}\left|\mu_{\nu}\right\rangle\left\langle\mu_{\nu}\left|U_{\mathbf{k}}\right| \eta_{0}\right\rangle
$$

A transição vibracional mediada pelo acoplamento do pósitron, a través do operador $U_{\mathbf{k}}$ depende do modelo que seja proposto.[23, 56] O grupo de pesquisa já utilizou a aproximação de ordem zero (Aproximação de Condon) para o operador $U_{\mathbf{k}}$ focando sua atenção em que o operador $\left(E-T_{N}-V_{\text {opt }}\right)^{-1}$ pode induzir acoplamentos entre o canal de entrada da molécula isolada e vários estados vibracionais do composto. Este texto tomará transições de primeira ordem em $U_{\mathbf{k}}$ definido via $V_{\text {cap }}$, ou seja, do estado fundamental até o primeiro estado excitado.

A amplitude de transição entre estados da molécula isolada $0 \rightarrow 1$ para um valor de energia $\left(k^{2} / 2\right)$ do pósitron, dada pela integral $\left\langle 1\left|U_{k}\right| \eta_{0}\right\rangle$, pode ser obtida desde os cálculos de espectroscopia vibracional como foi apresentado no capítulo anterior, utilizando o operador de polarizabilidade $(\langle 1|\alpha| 0\rangle)$ ou o momento dipolar $(\langle 1|\mu| 0\rangle)$, dependendo do tipo de transição vibracional (Raman ou IR-ativa). Dessa maneira, a partir da equação (6.6), fazemos a decomposição:

$$
\left\langle\mu_{\nu}\left|U_{k}\right| \eta_{0}\right\rangle=\sum_{\nu^{\prime}}\left\langle\mu_{\nu} \mid \eta_{\nu}^{\prime}\right\rangle\left\langle\eta_{\nu}^{\prime}\left|U_{k}\right| \eta_{0}\right\rangle \approx C_{\mu_{\nu}, 1}\left\langle 1\left|U_{k}\right| \eta_{0}\right\rangle
$$

onde o fator $C_{\mu_{\nu}, 1}$ é a amplitude de Franck-Condon[57] entre o primeiro estado excitado da molécula neutra $|1\rangle$ e os auto-estados do composto pósitron-molécula $\left\{\mu_{\nu}\right\}$. Usando a equação (6.7), a expressão (6.6) pode ser escrita da maneira:

$$
|\xi\rangle=\sum_{\mu}\left(E-T_{N}-V_{\mathrm{opt}}\right)^{-1}\left|\mu_{\nu}\right\rangle C_{\mu_{\nu}, 1}\left\langle 1\left|U_{k}\right| \eta_{0}\right\rangle
$$

O fato importante nessa maneira de ver o acoplamento do pósitron é que, uma vez que temos descrito o sistema ressonante na base de $\left\{\mu_{\nu}\right\}$, o operador $\left(E-T_{N}-V_{\text {opt }}\right)^{-1}=$ $\left(E-T_{N}-V^{\prime}+\frac{i}{2} \Gamma\right)$, nas aproximações feitas até agora, é diagonal de maneira que a 
equação (6.6) fica simplesmente:

$$
|\xi\rangle=\sum_{\mu} \frac{C_{\mu_{\nu}, 1}}{\left(\frac{k^{2}}{2}+\varepsilon_{\eta_{0}}-\varepsilon_{\mu_{\nu}^{\prime}}+\frac{i}{2} \Gamma\right)}\left|\mu_{\nu}\right\rangle\left\langle\eta_{1}\left|U_{k}\right| \eta_{0}\right\rangle
$$

onde $\varepsilon_{\mu_{\nu}}$ é a energia do estado vibracional do nivel $\nu$ do composto que satisfaz $\varepsilon_{\mu_{\nu}}=$ $\varepsilon_{\eta_{\nu}}+\varepsilon_{b}$

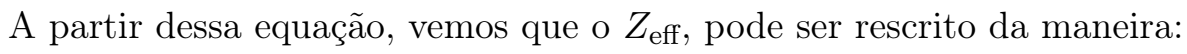

$$
Z_{\text {eff }}=\frac{2 \pi^{2}}{k} \rho_{d} \sum_{\mu} \frac{g_{\nu} \Gamma\left|C_{\mu_{\nu}, 1}\right|^{2}}{\left(\frac{k^{2}}{2}+\varepsilon_{\eta_{0}}-\varepsilon_{\mu_{\nu}}\right)^{2}+\left(\frac{\Gamma}{2}\right)^{2}}
$$

Vemos que, mesmo sendo um sistema com um modo normal, o espectro de $Z_{\text {eff }}$ pode estar composto por vários picos cuja altura é aumentada ou diminuída pelo fator de Franck-Condon $\left|C_{\mu_{\nu}, 1}\right|^{2}$. A soma de picos pode ser maior se incluimos todos os níveis do operador $U_{\mathbf{k}}$, no entanto vamos focar no estudo de primeira ordem que é um aporte original do trabalho.

Conhecendo sua utilidade, foram calculados os fatores de Franck-Condon $\left(\left|C_{\mu_{\nu}, 1}\right|^{2}\right)$ entre o estado $\left|\eta_{1}\right\rangle$ da molécula $\mathrm{LiH}$ e $\left|\mu_{\nu}\right\rangle$ do composto e $+\mathrm{LiH}$ a partir dos auto-estados do Hamiltoniano construído nos dois níveis de cálculo estudados. Os resultados são apresentados na tabela 6.2.

\begin{tabular}{lcr} 
Estado final $\left(\mu_{\nu}\right)$ & $\left|C_{\mu_{\nu}, 1}\right|^{2}($ UHF-MP2) & $\left|C_{\mu_{\nu}, 1}\right|^{2}(\mathrm{MRD}-\mathrm{CI})$ \\
\hline 0 & 0.033 & 0.254 \\
1 & 0.884 & 0.234 \\
2 & 0.081 & 0.306 \\
3 & 0.003 & 0.140 \\
4 & $6 \times 10^{-5}$ & 0.046 \\
5 & $4 \times 10^{-6}$ & 0.013 \\
\hline
\end{tabular}

TABEla 6.2: Coeficientes de Franck-Condon $\left|C_{\mu_{\nu}, 1}\right|^{2}=\left|\left\langle\mu_{\nu} \mid 1\right\rangle\right|^{2}$ para diferentes estados finais $\left(\mu_{\nu}\right)$ obtidos com as auto funções dos Hamiltonianos vibracionais construídos com as SEP do e+LiH obtidas nos níveis (UHF-MP2) e(MRD-CI)

Pode ser observado que, ao considerar o nível MRD-CI de cálculo, os coeficientes de Frack-Condon têm valores maiores a 0.03 para acoplamentos com os primeiros 5 níveis vibracionais do composto $\left\{\left|\mu_{\nu}\right\rangle \mid\right\}$ enquanto que no nível NEO-MP2, tais coeficientes são maiores do que 0.03 só nos primeiros 3 níveis vibracionais. Por tanto, enquanto a correlação elétron-pósitron leve a modific c oes maiores da geometria de equilibrio da molécula, os auto-estados do composto $\left\{\mu_{\nu}\right\}$ poderão ser visto como uma combinação maior de autoestados da molécula isolada $\left\{\nu_{\nu}\right\}$ e viceversa.

Também vale a pena observar que o acoplamento dos níveis vibracionais induzido pela correlação elétron-pósitron é apreciável principalmente com os níveis vibracionais vizinhos 
ou seja, vão ser acoplados os primeiros modos sendo pouco significativos os acoplamentos com overtones altos. Este fenômeno pode ser visto como um acoplamento seletivo, esboçado como um argumento para que não fosse sobre-estimado $Z_{\mathrm{eff}}$ se é suposto um acoplamento completo entre todos os níveis vibracionais quando lida-se com moléculas maiores. Nos modelos estudados até agora, no existe uma teoria satisfatória que faça uma estimativa adequada da maneira na que era feita a seleção no acoplamento[9] e tudo era colocado a mão.

É possível que o modelo apresentado para um nível vibracional possa ser generalizado para sistemas com mais modos normais de maneira que o operador $\left(E-T_{N}-V_{\text {opt }}\right)^{-1}$ pode fazer acoplamentos não somente entre overtones dos estados vibracionais mas entre modos normais da molécula isolada e os do composto. No seguinte capítulo, tentaremos apresentar uma maneira de calcular esse efeito para sistemas com diferentes modos normais de vibração.

\subsubsection{Afinidade positrônica}

Segundo o modelo proposto, a definição de uma afinidade positrônica não fica bem definida uma vez que a captura do pósitron acopla, em princípio, diversos níveis vibracionais. $O$ deslocamento de $Z_{\text {eff }}$ com respeito às linhas espectrais, vai ser dado pela diferencia da diferencia $\varepsilon_{\eta_{\nu}}-\varepsilon_{\mu_{\nu}}$ de maneira que cada pico de $Z_{\text {eff }}$ pode estar deslocado por uma quantidade diferente dependendo da transição vibracional excitada.

Para dar um número que, simultaneamente, indique que são possíveis excitações de diferentes níveis vibracionais $\mu_{\nu}$ do composto no acoplamento continuo-discreto do pósitron uma vez eles estão acoplados com o canal de entrada $\left|\eta_{0}\right\rangle$ pela correlação elétron-pósitron, é útil a definição da afinidade positrônica dada por:

$$
P A=\frac{\sum_{\nu}\left\langle\eta_{0}\left|U_{\nu}^{*}\left(H_{0}-H^{\prime}\right) U_{\nu}\right| \eta_{0}\right\rangle}{\sum_{\nu}\left\langle\eta_{0}\left|U_{\nu}^{*} U_{\nu}\right| \eta_{0}\right\rangle}
$$

onde, se somente são consideradas transições vibracionais de primeira ordem $0 \rightarrow 1$ induzidas por $U_{\nu}$, vemos que pode ser escrita simplesmente:

$$
P A \approx\left\langle 1\left|\left(H_{0}-H^{\prime}\right)\right| 1\right\rangle=\varepsilon_{1}-\sum_{\nu}\left|C_{\mu_{\nu}, 1}\right|^{2} \varepsilon_{\mu_{\nu}}
$$

onde $H_{0}$ é o hamiltoniano da molécula isolada com auto-estados $\left\{\eta_{\nu}\right\}$ e auto-energia $\varepsilon_{\eta_{\nu}}$ e $H^{\prime}$ é o hamiltoniano do sistema pósitron-molécula com auto-estados $\left\{\mu_{\nu}\right\}$ de energias $\varepsilon_{\mu_{\nu}}$. Se $H_{0}-H^{\prime}=\varepsilon_{b}$, então $\left|C_{\mu_{\nu}, 1}\right|^{2}=\delta_{\mu_{\nu}, 1}$ e $P A=-\left|\varepsilon_{b}\right|$ da maneira obtida no modelo de Gribakin-Lee. Os cálculos de este tipo de afinidade positrônica (PA) comparados com 
a diferencia dos mínimos de energia para a molécula e o composto $\left(\varepsilon_{b}\right)$, são apresentados na tabela 6.3

\begin{tabular}{lcr} 
Molécula & PA $(\mathrm{meV})$ & $\varepsilon_{b}(\mathrm{meV})$ \\
\hline LiH (MP2) & 27.9 & 26.3 \\
LiH (MRD-CI) & 80.7 & 79.5 \\
\hline NaH (MP2) & 45.1 & 43.4 \\
NaH (MRD-CI) & 105.9 & 103.5 \\
\hline
\end{tabular}

TABEla 6.3: Afinidade positrônica (PA) e diferencia de energia dos mínimos para as moléculas de $\mathrm{LiH}$ e $\mathrm{NaH}$ no nível de cálculo MRD-CI

é possível observar que, em geral, as afinidades positrônicas, da maneira definida na equação (6.12) são maiores, em valor absoluto, do que a diferencia de energias nos mínimos $\left(\varepsilon_{b}\right)$ uma vez que contemplam, simultâneamente, a diminuída de concavidade na CEP. No entanto, utilizar $\varepsilon_{b}$ é uma boa aproximação e um número mais simples de calcular.

\subsubsection{Transições permitidas}

Os acoplamentos vibracionais do estado $|1\rangle$ com os estados $\left\{\left|\mu_{\nu}\right\rangle\right\}$ permite que seja possível um conjunto de transições vibraiconais sempre que elas satisfazer a conservação da energia:

$$
\frac{k^{2}}{2}+\varepsilon_{0}=\varepsilon_{\mu_{\nu}}
$$

sendo $k^{2} / 2$ a energia cinética do pósitron incidente, $\varepsilon_{0}$ a energia do estado fundamental da molécula isolada e $\varepsilon_{\mu_{\nu}}$ a energia auto-estado vibracional $\left|\mu_{\nu}\right\rangle$ do composto pósitronmolécula.

Portanto, já que é definido $k^{2} / 2>0$, segundo (6.13), toda vez que $\varepsilon_{\mu_{\nu}}-\varepsilon_{0}<0$ a transição não pode ser feita por um pósitron acoplado ao contínuo. Sob essa premissa, somente serão vibracionalmente ativas, pelo acoplamento do pósitron, moléculas com baixa afinidade positrônica e transições vibracionais com energia relativamente alta. Segundo isto, poderiam ser acoplados níveis vibracionais altos do composto no entanto, como foi observado nas tabelas 6.2 e 6.3 para eles, os coeficientes de Franck-Condon com $|1\rangle$ são praticamente nulos e por tanto, os picos em $Z_{\text {eff }}$ dados por essas transições, são imperceptíveis.

Tal efeito já tem sido observado experimentalmente. Por exemplo, não acontecem ressonâncias vibracionais de Feshbach perceptíveis para Alcanos de afinidades positrônicas maiores do que a energia típica da transição vibracional do $\mathrm{C}-\mathrm{H}(\approx 380 \mathrm{meV})$ sendo o limite o Hexadecano $\left(\mathrm{C}_{16} \mathrm{H}_{34}\right)$ com afinidade positrônica $\approx 310 \mathrm{meV}$ [43]. Dessa maneira, uma vez que $\varepsilon_{b} \approx 1 \mathrm{eV}$ para o $\mathrm{LiH}$ e é muito maior do que a energia de suas transições típicas $(\approx 0.17 \mathrm{eV})$, não será possível um acoplamento do pósitron nela. 


\subsection{Conclusões}

Neste capítulo foi desenvolvido o cálculo de curvas de energia potencial (CEP) para os sistemas $\mathrm{LiH}, \mathrm{e}+\mathrm{LiH}$ e $\mathrm{NaH}$ e $+\mathrm{NaH}$ nos níveis MCD-CI e MP2. Usando os dois níveis de cálculo, observamos que existe uma variação importante da CEP ao considerar a correlação elétron-pósitron com este último, ligado á molécula. Tal correlação permite acoplar uma boa quantidade de níveis vibracionais da molécula isolada. No nível MP2, existe um acoplamento apreciável do primeiro modo excitado da molécula de LIH com os primeiros 3 níveis vibracionais do e+LiH e no nível MRD-CI, um acoplamento apreciável com os 5 primeiros. Foi observado, no entanto, que as moléculas de $\mathrm{LiH}$ e $\mathrm{NaH}$, não apresentarão ressonância vibracionais de Feshbach uma vez que a afinidade positrônica de cada uma é maior do que a energia das transições vibracionais típicas e o acoplamento vibracional com overtones muito altos não é estimulado pela correlação elétron-pósitron. 


\section{Capítulo 7}

\section{Acoplamentos vibracionais intermediados pela correlação elétron-pósitron para moléculas com mais de um modo normal.}

\subsection{Introdução}

No capitulo anterior, foi apresentado o procedimento para resolver o estado vibracional total, conhecendo os auto estados associados à SEP da molécula isolada e os associados à SEP do composto construída levando em conta a correlação pósitron-molécula. Esse estado vibracional pode ser composto por uma grande quantidade de autoestados do composto dependendo da maneira na que eles são acoplados com o estado de entrada. Foi discutido o caso para um modo vibracional chegando á conclusão de que quando a diferencia entre as duas SEPs é suficientemente grande, a quantidade desse acoplamentos pode ser importante.

Neste capítulo, a diferencia entre as SEP será modelado de maneira perturbativa e na aproximação harmónica. O caráter perturbativo é justificado uma vez que esperamos que as moléculas que consigam acoplar o pósitron apresentem, também, uma mudança pequena da sua SEP. Uma mudança grande da SEP está associada, quase sempre, a um deslocamento grande da energia da geometria de equilíbrio de maneira que se esta excede a energia das transições vibracionais $(\sim 500 \mathrm{meV})$, é muito difícil acoplar vibracionalmente ao pósitron como foi observado nas moléculas de hidretos. A aproximação 
harmônica, além disso, permitirá descrever as contribuições perturbativas ao hamiltoniano em termos de operadores criação e destruição permitindo o cálculo analítico dos auto-estados do composto a primeira ordem perturbativa.

A aproximação perturbativa, também vem de que computacionalmente, lidaremos com a dificuldade de que atualmente, existem programas otimizados para o cálculo da matriz Hessiana (a aproximação harmônica da SEP) apenas para sistemas de elétrons. De maneira que não são possíveis, para sistemas de dois ou mais modos vibracionais, nem o cálculo e nem muito menos a diagonalização da matriz Hessiana do composto pósitronmolécula.

Nesta seção, a partir dos cálculos de estrutura eletrônica no nível MP2, será estimada a SEP de uma molécula com mais de um modo vibracional e aproximada a operadores harmônicos. Para moléculas de três átomos (3 modos vibracionais ou 4 se é linear), não têm sido reportadas ressonâncias vibracionais de Feshbach uma vez que uma grande parte de elas não consegue ligar ao pósitron[43]. O HCN é uma molécula simples que, segundo os cálculos computacionais, tem uma afinidade positrônica grande dado seu apreciável momento dipolar (3.312 D) mas que possui problemas de manipulação nos experimentos. No nível de cálculo FN-DMC, sua afinidade positrônica é $\varepsilon_{b} \approx 0.038 \mathrm{eV}$ [58]. Espera-se que no nível de cálculo MP2, seja muito menor mas suficiente para fazer algumas estimativas e apresentar o método.

\subsection{Mecanismo de transição para mais de um modo normal}

No capítulo anterior, foi mostrado cómo, conhecendo os auto-estados vibracionais da molécula isolada $\left\{\left|\eta_{\nu}\right\rangle\right\}$ e os auto-estados vibracionais do composto $\left\{\left|\mu_{\nu}\right\rangle\right\}$, é possível calcular o espectro de $Z_{\text {eff }}$ incluído os níveis vibracionais do composto accessíveis a través de um canal de entrada dado. Cada pico de $Z_{\text {eff }}$ associado a uma transição $\eta_{0} \rightarrow \mu_{\nu}$ vai ter um tamanho relativo dado pelo fator de Frack-Condon:

$$
\left|\left\langle\mu_{\nu}\left|U_{k}\right| \eta_{0}\right\rangle\right|^{2}
$$

o qual, considerando unicamente a primeira ordem de $U_{k}$, fica:

$$
\left|\left\langle\mu_{\nu} \mid 1\right\rangle\left\langle 1\left|U_{k}\right| \eta_{0}\right\rangle\right|^{2}=\left|C_{\mu_{\nu}, 1}\right|^{2}|\Gamma|
$$

Para o caso geral, no qual os estados $\left\{\left|\eta_{\nu}\right\rangle\right\}$ vão ser combinações de varios auto estados do composto $\mu_{\nu}$, de maneira que os fatores de Franck-Condon são definidos como a norma ao quadrado dos coeficientes da matriz de transformação que define os estados vibracionais 
do composto em termos dos estados da molécula isolada

$$
\left|\mu_{\nu}\right\rangle=\sum_{\eta_{\nu}} C_{\mu_{\nu}, \eta_{\nu}}\left|\eta_{\nu}\right\rangle
$$

Asim, se o canal vibracional de entrada é o primeiro estado excitado do modo vibracional $\nu$ da molécula isolada $\left(\left|1_{\nu}\right\rangle\right)$ os fatores de Franck-Condon importantes são $C_{\mu_{\nu}, 1_{\nu}}$ de

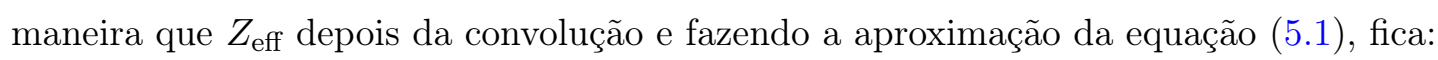

$$
Z_{\mathrm{eff}}(\epsilon)=\frac{2 \pi^{2}}{k_{\nu}} \rho_{d} \sum_{\nu}\left|C_{\mu_{\nu}, 1_{\nu}}\right|^{2} g_{\nu} \Delta\left(\epsilon-\varepsilon_{\mu_{\nu}}\right)
$$

Observamos que, no caso geral de vários modos vibracionais, ao incluir os fatores de Franck-Condon poderiamos aumentar o diminuir alguns picos do espectro de $Z_{\text {eff }}$ em relação aos calculados na aproximação de GL. Esse efeito é conhecido nos resultados experimentais sendo modelado de uma maneira empírica [59]:

$$
Z_{\mathrm{eff}}(\epsilon)=\pi F \sum_{\nu} \beta_{\nu} \sqrt{\frac{\epsilon_{b}}{\epsilon_{\nu}}} g_{\nu} \Delta\left(\epsilon_{\nu}-\epsilon\right)
$$

com $\beta_{\nu}$ corresponde a um parâmetro empírico. $\beta_{\nu}>1$ se o pico é mais alto do que o esperado na teoria GL, $\beta_{\nu}<1$ se são menores e $\beta=1$ se são iguais. O termo adicional $\sqrt{\epsilon_{b} / \epsilon_{\nu}}$ também é introduzido a $Z_{\text {eff }}$ uma vez $\rho_{d} \approx(F / 2 \pi) \sqrt{\epsilon_{b}} \operatorname{com} F=0.66 \mathrm{e}$ $k_{\nu}=\sqrt{\epsilon_{\nu}}[60]$. Assim, podemos propor a equivalência:

$$
\beta_{\nu}=\left|C_{\mu_{\nu}, 1_{\nu}}\right|^{2}
$$

\subsection{Aproximação harmónica}

Considerando a molécula como um sistema de vários núcleos interagentes sob um potencial efetivo dos elétrons no sentido Born-Oppenheimer, a aproximação harmônica da SEP permite considerar á molécula inteira como osciladores harmônicos desacoplados que podem ser descritos com coordenadas normais $\left\{Q_{k}\right\}$ que diagonalizam esse Hamiltoniano. A diferencia entre a SEP da molécula com o pósitron $\left(V_{\mu}(\mathbf{Q})\right)$ e a SEP da molécula isolada $\left(V_{0}(\mathbf{Q})\right)$ pode, mantendo a aproximação harmônica, ser escrita da forma:

$$
\bar{\varepsilon}(\mathbf{Q})=V_{\mu}(\mathbf{Q})-V_{0}(\mathbf{Q})=\bar{\varepsilon}(0)+\nabla \bar{\varepsilon}(0) \cdot \mathbf{Q}+\frac{1}{2} \mathbf{Q} \cdot \nabla^{2} \bar{\varepsilon}(0) \cdot \mathbf{Q}+\cdots
$$


de maneira que o operador $T_{N}+V_{\text {opt }}$ pode ser expressado como o hamiltoniano de um conjunto de osciladores acoplados mais termos de amortecimento constantes:

$$
T_{N}+V_{o p t}=-\varepsilon_{b}+\sum_{k} \frac{P_{k}^{2}}{2 m_{k}}+\frac{1}{2} \omega_{k}^{2} Q_{k}^{2}-F_{k} Q_{k}+\frac{1}{2} \sum_{k, l} K_{k l} Q_{k} Q_{l}-\frac{i}{2} \sum_{k} \Gamma_{k}
$$

Temos definido $F_{k}=-\partial_{Q_{k}} \bar{\varepsilon}$ e $K_{k l}=\partial_{Q_{k} Q_{l}}^{2} \bar{\varepsilon}$.

Para compostos pósitron-molécula o problema consiste no cálculo de $\bar{\varepsilon}$ de uma maneira eficiente e em consequência, o cálculo de $F_{k}$ e $K_{k l}$. No trabalho as derivadas vão ser feitos praticamente a mão calculando ponto por ponto $\bar{\varepsilon}$ para algumas coordenadas de cada modo normal.

A aproximação perturbativa vai ser válida dependendo do valor relativo de $F_{k}$ e $K_{k l}$. A ordem zero de perturbações corresponde ao modelo de Gribakin-Lee(7.8). Esse modelo, só leva em conta o deslocamento na energia $\left(\bar{\varepsilon}(0)=-\varepsilon_{b}\right)$ sem termos harmônicos adicionais. Tal modelo, consegue prever o deslocamento das linhas espectrais de $Z_{\text {eff }}$ mas não leva às observadas acoplamentos entre modos vibracionais adicionais que, podem ser obtidas ao considerar mudanças na concavidade da SEP $\left(K_{k l}\right)$ e na geometria de equilíbrio do sistema com respeito a os da molécula isolada $\left(F_{k}\right)$.

Uma construção mais simples para lidar com o problema perturbativo consiste em utilizar o formalismo de operadores criação e destruição. Definindo os operadores (em unidades atômicas)

$$
\begin{aligned}
c_{k} & =\sqrt{\frac{m_{k} \omega_{k}}{2}}\left(Q_{k}+\frac{i}{m_{k} \omega_{k}} P_{k}\right) \\
c_{k}^{\dagger} & =\sqrt{\frac{m_{k} \omega_{k}}{2}}\left(Q_{k}-\frac{i}{m_{k} \omega_{k}} P_{k}\right)
\end{aligned}
$$

é possível reescrever a equação (7.8) da maneira:

$T_{N}+V_{\mathrm{opt}}=-\varepsilon_{b}+\sum_{k}\left\{\omega_{k}^{\prime}\left(\eta_{k}+1 / 2\right)+\Omega_{k}\left(c_{k}+c_{k}^{\dagger}\right)\right\}+\sum_{k, l} \omega_{k, l}\left(c_{k}^{\dagger} c_{l}^{\dagger}+c_{k}^{\dagger} c_{l}+c_{k} c_{l}^{\dagger}+c_{k} c_{l}\right)-\frac{i}{2} \sum_{k} \Gamma_{k}$

onde $\omega_{k}^{\prime}=\omega_{k} \sqrt{1+\epsilon_{k}}, \epsilon_{k}=\frac{K_{k k}}{\omega_{k}^{2} m_{k}}, \Omega_{k}=F_{k} \sqrt{\frac{1}{2 m_{k} \omega_{k}}}$ e $\omega_{k, l}=\frac{K_{k l}}{2\left(\omega_{k} \omega_{l} m_{k} m_{l}\right)^{1 / 2}}$. Dessa maneira é conluído que o regime perturbativo é satisfeito toda vez que $K_{k k}<<\omega_{k}^{2} m_{k}$, $\Omega_{k}<<\omega_{k}$ e $\omega_{k l}<<\left(\left|\omega_{k}-\omega_{l}\right|\right)$.

\subsubsection{Construção da base de auto-estados do composto}

Uma vez descritos os operadores perturbativos na base de operadores de criação e destruição da molécula isolada $\left\{\left|\eta_{\nu}\right\rangle\right\}$, é simples aplicar a teoria de perturbações de primeira 
ordem para obter a base de auto-estados vibracionais do composto $\left\{\left|\mu_{\nu}\right\rangle\right\}$. A maneira de exemplo, é apresentado o caso no qual o sistema pode ser descrito por dois modos vibracionais não degenerados:

$$
\begin{aligned}
\left|\mu_{k}, \mu_{l}\right\rangle=\quad & \left|\eta_{k}, \eta_{l}\right\rangle \\
& -\frac{\epsilon_{k}}{4 \sqrt{2}}\left(\left|\eta_{k+2}, \eta_{l}\right\rangle+\left|\eta_{k-2}, \eta_{l}\right\rangle\right)-\frac{\epsilon_{l}}{4 \sqrt{2}}\left(\left|\eta_{k}, \eta_{l+2}\right\rangle+\left|\eta_{k}, \eta l+2\right\rangle\right)+ \\
& \frac{\Omega_{k}}{\omega_{k}}\left(\left|\eta_{k+1}, \eta_{l}\right\rangle+\left|\eta_{k-1}, \eta_{l}\right\rangle\right)+\frac{\Omega_{l}}{\omega_{l}}\left(\left|\eta_{k}, \eta_{l+1}\right\rangle+\left|\eta_{k}, \eta_{l-1}\right\rangle\right)+ \\
& \omega_{k l \mu}\left(\frac{\left|\eta_{k+1}, \eta_{l+1}\right\rangle}{\omega_{\eta_{k+1}}-\omega_{\eta_{l+1}}}+\frac{\left|\eta_{k+1}, \eta_{l-1}\right\rangle}{\omega_{\eta_{k+1}}-\omega_{\eta_{l-1}}}+\frac{\left|\eta_{k-1}, \eta_{l+1}\right\rangle}{\omega_{\eta_{k-1}}-\omega_{\eta_{l+1}}}+\frac{\left|\eta_{k-1}, \eta_{l-1}\right\rangle}{\omega_{\eta_{k-1}}-\omega_{\eta_{l-1}}}\right)
\end{aligned}
$$

onde $\omega_{\eta_{k}}=\omega_{0_{k}}(k+1 / 2)$ é a frequência do modo vibracional $k$ no estado dado pelo número quântico $\eta_{k}=0,1,2 \cdots$, obtida a partir da sua frequência fundamental $\left(\omega_{0_{k}}\right)$. Aqui, embora a molécula de HCN apresenta dois modos degenerados, a inclusão do pósitron não quebra a simetria do sistema e os estados do composto continuam sendo degenerados de maneira que $\omega_{k l}=0$ para $k$ e $l$ modos degenerados do mesmo autoestado de energia.

A expansão dos estados vibracionais do composto na base de estados vibracionais da molécula isolada, permite a o cálculo das amplitudes de Franck-Condon explicadas pela definição (7.3). Dessa maneira, por exemplo, a amplitude entre o estado $\left|\eta_{k}, \eta_{l}\right\rangle$ da molécula isolada e o estado $\left|\mu_{i}, \mu_{l}\right\rangle$ do composto, pode ser escrita da maneira:

$$
\begin{aligned}
\left\langle\eta_{k}, \eta_{l} \mid \mu_{k}, \mu_{l}\right\rangle=\quad & \delta_{\eta_{k}, \mu_{k}} \delta_{\eta_{l}, \mu_{l}} \\
& -\frac{\epsilon_{k}}{4 \sqrt{2}}\left(\delta_{\eta_{k}, \mu_{k}+2}+\delta_{\eta_{k}, \mu_{k}-2}\right) \delta_{\mu_{l}, \eta_{l}}-\frac{\epsilon_{l}}{4 \sqrt{2}}\left(\delta_{\eta_{l}, \mu_{l}+2}+\delta_{\eta_{l}, \mu_{l}-2}\right) \delta_{\mu_{k}, \eta_{k}}+ \\
& \frac{\Omega_{k}}{\omega_{k}}\left(\delta_{\eta_{k}, \mu_{k}+1}+\delta_{\eta_{k}, \mu_{k}-1}\right) \delta_{\mu_{l}, \eta_{l}}+\frac{\Omega_{l}}{\omega_{l}}\left(\delta_{\eta_{l}, \mu_{l}+1}+\delta_{\eta_{l}, \mu_{l}-1}\right) \delta_{\mu_{k}, \eta_{k}}+ \\
& \omega_{k l}\left(\frac{\delta_{\eta_{k}, \mu_{k}+1} \delta_{\eta_{l}, \mu_{l}+1}}{\omega_{\eta_{k}+1}-\omega_{\eta_{l}+1}}+\frac{\delta_{\eta_{k}, \mu_{k}+1} \delta_{\eta_{l}, \mu_{l}-1}}{\omega_{\eta_{k}+1}-\omega_{\eta_{l}-1}}+\frac{\delta_{\eta_{k}, \mu_{k}-1} \delta_{\eta_{l}, \mu_{l}+1}}{\omega_{\eta_{k}-1}-\omega_{\eta_{l}+1}}+\frac{\delta_{\eta_{k}, \mu_{k}-1} \delta_{\eta_{l}, \mu_{l}-1}}{\omega_{\eta_{k}-1}-\eta_{\eta_{l}-1}}\right)
\end{aligned}
$$

onde $\delta_{\mu_{k}, l}$ é o símbolo delta de Kronecker. A partir do resultado da equação (7.12) podemos observar que as mudanças na concavidade na SEP $\left(\epsilon_{\eta}\right)$ preservam a paridade da função de onda e permitem acoplar estados excitados $\eta_{k}$ e $\eta_{k}^{\prime}$ de um modo $k$ seguindo a regra de seleção $\left|\eta_{k}-\eta_{k}^{\prime}\right|=2$. No entanto, tal acoplamento é inversamente proporcional a $\omega_{\eta}^{2}$ de maneira que para modos com frequência fundamental maior, este tipo de efeitos vão ser menores.

As mudanças na geometria de equilíbrio, representadas principalmente pelos termos gradientes $\left(\Omega_{k}\right)$, conseguem fazer transições entre estados excitados $\eta_{k}$ e $\eta_{k}^{\prime}$ do modo $k$ que satisfazem a regra: $\left|\eta_{k}-\eta_{k}^{\prime}\right|=1$. Tal acoplamento é proporcional a $\omega_{k}^{-3 / 2}$ de maneira que 
poderá ser ainda apreciável para transições com frequência fundamental $\omega_{k}$ relativamente grande.

Os elementos intermodais da SEP $\left(\omega_{k l}\right)$,poderão acoplar, apreciavelmente, estados com energias similares mesmo eles sejam de modos normais diferentes. Eventualmente, se as energias dos modos acoplados são suficientemente parecidas, o acoplamento vai ser muito forte.

Conhecendo, portanto, a maneira na qual cada operador, definido na aproximação harmônica da deformação da SEP induzida pela correlação pósitron-elétron, atua sobre os autoestados vibracionais, é possível uma estimativa das correções ao espectro de aniquilação. Um calculo de esses operadores, será apresentada na seguinte seção.

\subsection{Aplicação da teoria à molécula de $\mathrm{HCN}$}

O primeiro passo realizado para o cálculo das correções da SEP da HCN, foi definir as coordenadas do sistema. Na literatura, o maior nível de cálculo empregado para obter a geometria de equilíbrio da molécula de HCN é o CISD[61]: nele, para a molécula isolada, o comprimento da ligação H-C é de $1.066 \AA$, o comprimento do C-N é de $1.167 \AA$ e sua afinidade positrônica definida como a diferença entre a energia do e $+\mathrm{HCN}$ e a energia do HCN sem alterar a geometria é $18.3 \mathrm{meV}$. Os cálculos melhor convergidos mas sem geometria optimizada correspondem ao nível FN-DMC. A energia, para ambos compostos, é calculada na geometria optimizada no nível CISD usando a base de Gaussianas $6-311++\mathrm{G}(3 \mathrm{~d}, 2 \mathrm{p})$ e a base positrônica [15s15p6d2f]. A partir dos valores de energia é prevista uma afinidade de 37.8(4.8) $\mathrm{meV}$.

Neste trabalho, foi obtida a geometria de equilíbrio da molécula isolada no nível MP2 com bases eletrônicas $6-311 \mathrm{G}^{++}(2 p, 2 d): \mathrm{H}-\mathrm{C}=1.069 \AA$ e $\mathrm{C}-\mathrm{N}=1.171 \AA$. Com métodos computacionais usuais, apresentado brevemente no apêndice B.1, foram calculados os modos normais $\left(\mathbf{Q}_{i}\right)$ e a massas reduzidas de cada modo $\left(m_{i}\right)$.

Foram encontrados três modos de vibração: dois longitudinais: o associado, predominantemente, a o deslocamento $\mathrm{C}-\mathrm{H}$ com $\omega_{1}=250 \mathrm{meV}$ designado como modo 1 e o associado ao deslocamento $\mathrm{C} \equiv \mathrm{N}$ com $\omega_{2}=430 \mathrm{meV}$ designado como modo 2 e um transversal com degenerescência 2 e com $\omega_{3}=86 \mathrm{meV}$ designado como modo 3. As energias eletrônicas no sentido Born-Oppenheimer foram calculadas no nível de cálculo UHF-MP2 com bases eletrônicas $6-311 \mathrm{G}^{++}(2 p, 2 d)$. 
No nível de cálculo MP2, utilizando a base positrônica $10 S 10 p 10 d$, foi calculada a energia do sistema para diferentes deslocamentos dos modos normais. Na figura 7.1b é apresentada a densidade de probabilidade do pósitron na geometria de equilibrio,

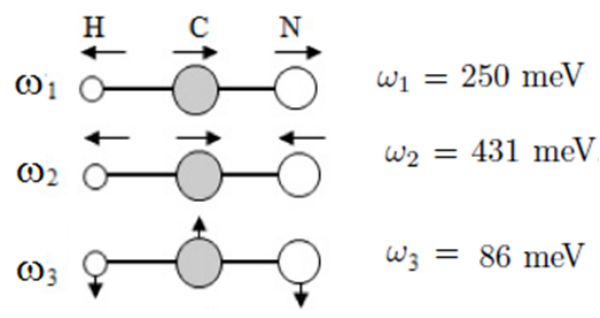

(A)

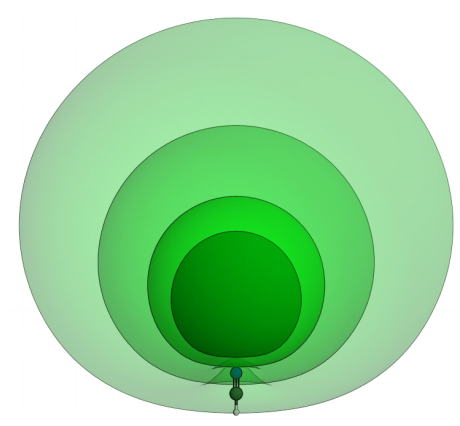

(B)

FigurA 7.1: (a) Representação gráfica dos modos normais da molécula de HCN (b) Densidade de probabilidade positrõnica para a molécula de HCN (b)Densidade de probabilidade positrõnica para a molécula de $\mathrm{HCN}$ para o $25 \%, 50 \%, 75 \%$ e $90 \%$ com base eletrônica 6-311++G(de,dp) é positrônica 10s10d10p no nível UHF-MP2.

Observamos que o pósitron vai ficar principalmente ao lado do átomo de Nitrogênio mas no nível de calculo empregado, encontra-se muito deslocalizado com respeito a o tamanho da molécula de maneira que espera-se que as contribuições da correlação elétron-pósitron para este estado praticamente deslocalizado, sejam muito menores do que as obtidas, possivelmente em níveis de cálculo maiores.

Os termos $K_{l k}$ e $F_{k}$ são obtidos a partir do cálculo de $\varepsilon(\mathbf{Q})=V_{m} u(\mathbf{Q})-V_{0}(\mathbf{Q})$ como derivada numérica para vários deslocamentos dos modos vibracionais $Q_{k}$ seguindo a relação:

$$
F_{k}=\frac{\partial \varepsilon}{\partial Q_{k}} \quad K_{l k}=\frac{\partial^{2} \varepsilon}{\partial Q_{k}^{2}}
$$

Para o cálculo numérico das derivadas (diferenças finitas), cada núcleo foi deslocado até uma geometria $\mathbf{R}_{i}$ obtida desde a geometria de equilíbrio $\mathbf{R}_{0}$ com um parâmetro $h$, vezes o vetor do modo normal $\mathbf{Q}_{i}$ satisfazendo:

$$
\mathbf{R}_{i}(t)=\mathbf{R}_{0}+h \mathbf{Q}_{i}
$$

A transformação (7.14) é um caso particular da transformação unitária que define um deslocamento e abordada no apêndice B.1.

Foi calculada a energia para vários deslocamentos desses modos normais permitindo o levantamento de alguns pontos da SEP do composto pósitron-molécula na base de coordenadas normais da molécula isolada. Para deslocamentos de modos normais puros, ou 
seja, um deslocamento de um modo mantendo os outros deslocados, foi feita a figura 7.2

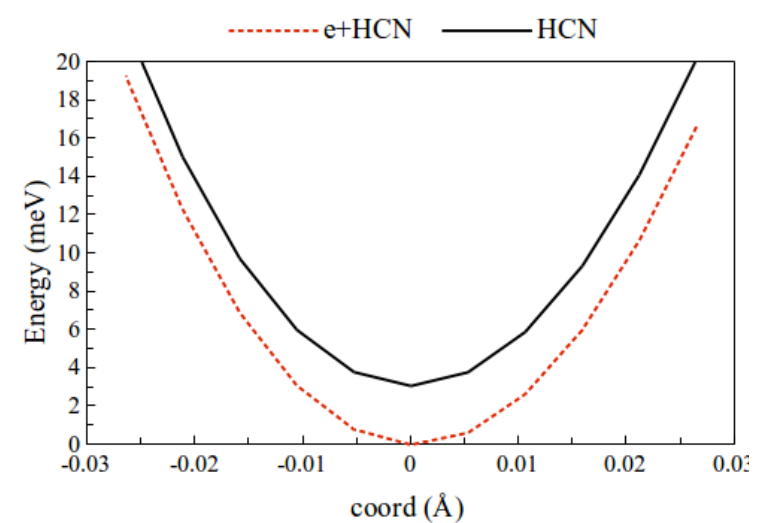

(A)

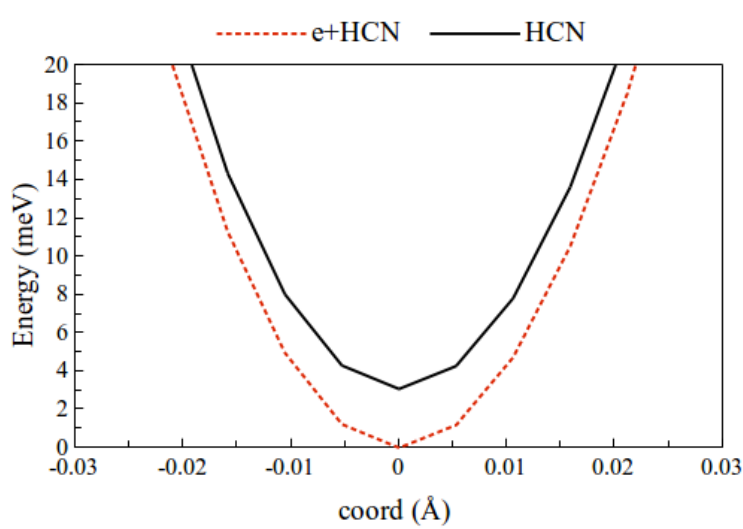

(B)

FigurA 7.2: Curvas de energia potencial nas coordenadas dos modos vibracionais longitudinais da molécula de $\mathrm{HCN}$ e o composto e+HCN calculadas no nível UHFNEO-MP2 (a) primeiro modo vibracional com frequência fundamental $\omega_{1}=250 \mathrm{meV}$ (b) Segundo modo vibracional com frequência fundamental $\omega_{2}=431 \mathrm{meV}$. Os níveis vibracionais não são apresentados uma vez que o modo mais baixo tem energia maior de $100 \mathrm{meV}$

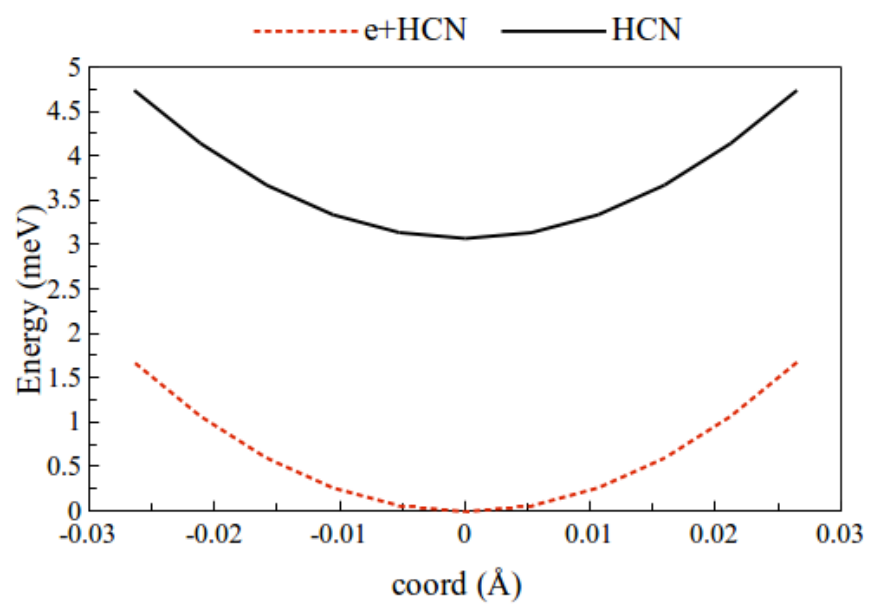

Figura 7.3: Curva de energia potencial nas coordenadas do modo vibracional transversal da molécula de $\mathrm{HCN}$ e o composto e $+\mathrm{HCN}$ cuja frequência fundamental é $\omega_{1}=$ $86 \mathrm{meV}$ calculada no nívelHF-NEO-MP2. Os níveis vibracionais não são apresentados uma vez que o modo mais baixo tem energia maior de $40 \mathrm{meV}$

Utilizando um deslocamento de $h=0.01 a_{0}$, foi utilizado o método de diferencias finitas para fazer uma estimativa da primeira $\left(F_{k}\right)$ e segunda $\left(K_{l k}\right)$ derivadas da superfície em torno a à geometria de equilíbrio pela fórmula: 


$$
\begin{aligned}
F_{k} & =\frac{\partial \varepsilon}{\partial Q_{k}} \approx \frac{\varepsilon\left(h Q_{k}, 0\right)-\varepsilon\left(-h Q_{k}, 0\right)}{2 h Q_{k}} \\
K_{l l} & =\frac{\partial^{2} \varepsilon}{\partial Q_{k}^{2}} \approx \frac{\varepsilon\left(h Q_{l}, 0\right)-2 \varepsilon(0,0)+\varepsilon\left(-h Q_{l}, 0\right)}{h^{2}}
\end{aligned}
$$

Onde $\varepsilon(a, b)$ é a energia na geometria com deslocamento $a$ no primeiro modo e deslocamento $b$ no segundo modo. Para calcular os termos cruzados $K_{l k}$ do potencial foram feitos cálculos da energia para geometrias correspondentes a deslocamentos simultâneos de dois modos normais $l$ e $k$. Assim, a derivada cruzada $\left(K_{l k}\right)$ é calculada da maneira[28]:

$$
\begin{aligned}
K_{l k} & =\frac{\partial^{2} \varepsilon}{\partial Q_{k} \partial Q_{l}} \\
& \approx \frac{\varepsilon\left(h Q_{l}, h Q_{k}\right)-\varepsilon\left(h Q_{l}, 0\right)-\varepsilon\left(0, h Q_{k}\right)+2 \varepsilon(0,0)-\varepsilon\left(-h Q_{l}, 0\right)-\varepsilon\left(0,-h Q_{k}\right)+\varepsilon\left(-h Q_{1},-h Q_{k}\right)}{2 h^{2}}
\end{aligned}
$$

Uma vez obtidos os valores $F_{k}$ e $K_{l k}$, foram calculados os valores das frequências $\Omega_{k}$ e $\omega_{l k}$, definidas na equação (7.10) para cada modo vibracional. Esses parâmetros são apresentados na tabela 7.1

\begin{tabular}{lccr} 
Termo & Frequência $(\mathrm{meV})$ & Termo & Frequência $(\mathrm{meV})$ \\
\hline$\omega_{1}$ & 250.00 & $\omega_{1}^{\prime}$ & 250.02 \\
$\omega_{2}$ & 431.41 & $\omega_{2}^{\prime}$ & 431.28 \\
$\omega_{3}$ & 86.20 & $\omega_{3}^{\prime}$ & 86.33 \\
$\omega_{1,2}$ & $1.79 \times 10^{-3}$ & $\omega_{1,2}^{\prime}$ & $2.41 \times 10^{-2}$ \\
$\omega_{1,3}$ & $1.32 \times 10^{-3}$ & $\omega_{1,3}^{\prime}$ & $2.35 \times 10^{-3}$ \\
$\omega_{2,3}$ & $8.23 \times 10^{-4}$ & $\omega_{2,3}^{\prime}$ & $8.29 \times 10^{-4}$ \\
$\varepsilon_{b}$ & 3.08 & & \\
$\Omega_{1}$ & $5.51 \times 10^{-2}$ & & \\
$\Omega_{2}$ & 1.033 & & \\
$\Omega_{3}$ & 0.00 & & \\
$\Gamma$ & $\sim 10^{-1}$ & &
\end{tabular}

TABela 7.1: Parâmetros da dinâmica vibracional da molécula e do composto, onde

$$
\omega_{k}^{\prime}=\omega_{k} \sqrt{1+\epsilon_{k}}, \epsilon_{k}=\frac{K_{k k}}{\omega_{k}^{2} m_{k}}, \Omega_{k}=F_{k} \sqrt{\frac{1}{2 m_{k} \omega_{k}}} \mathrm{e} \omega_{k, l}=\frac{K_{k l}}{2\left(\omega_{k} \omega_{l} m_{k} m_{l}\right)^{1 / 2}} .
$$

Para nosso caso particular, observamos que as correções na maioria de parâmetros são muito pequenas. A frequências fundamentais das vibrações apenas vão mudar alguns décimos de meV. As mais evidentes correções são as dos gradientes $\Omega_{k}$ sendo, a maior, de $1 \mathrm{meV}$. Os termos $\omega_{k, l}$ mostram que não existe acoplamento significativo entre os modos longitudinais e o modo transversal, uma vez que eles são da mesma ordem de grandeza dos acoplamentos calculados sem o pósitron correspondente à precisão numérica. $\mathrm{O}$ acoplamento entre modos longitudinais, $\omega_{1,2}$, cresce uma ordem de grandeza mas continua sendo desprezível com respeito às frequências típicas de vibração $\omega_{1}$ e $\omega_{2}(\sim 0.01 \%)$. Dessa maneira, nosso nível de cálculo, a contribuição das correções no Hamiltoniano é 
praticamente desprezível e, ao considerar somente o deslocamento na energia $\varepsilon_{\mu}=3.1$ meV, a aproximação de Gribakin-Lee é razoável.

No entanto, mantendo as proporções, é possível notar que os efeitos que vem com a ligação do pósitron não afetam da mesma maneira a todos os modos. Observamos qualitativamente dois efeitos importantes: primeiro, o pósitron vai permanecer perto do átomo de Nitrogênio de maneira que enlace $\mathrm{C} \equiv \mathrm{N}$ (modo 2) vai ser esticado uma quantidade maior do que nos outros enlaces de maneira que o modo vibracional associado à vibração de esse enlace é o mais afetado. As características dos modos transversal e associado a $\mathrm{C}-\mathrm{H}$, vão permanecer praticamente iguais antes e depois de ligar o pósitron.

Segundo, o modo transversal não está relativamente acoplado, com os outros modos, nem antes nem depois da inclusão do pósitron sendo seus acoplamentos da mesma ordem da precisão numérica. Existe, no entanto, um acoplamento relativamente importante entre os modos longitudinais depois da inclusão do pósitron. Numericamente, esse acoplamento é uma ordem de grandeza maior do que acoplamento sem pósitron mas continua sendo desprezível.

Com os parâmetros encontrados, foi feita um gráfico de $Z_{\text {eff }}$ apresentado na figura 7.4 Vemos que as correções não são muito importantes. Elas são muito pequenas, segura-

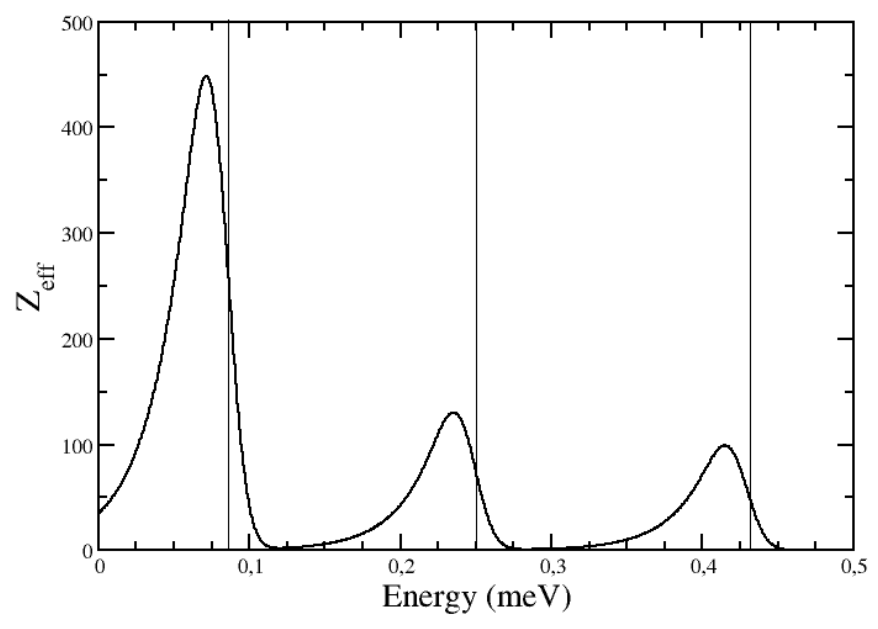

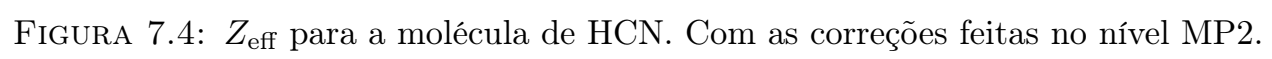

mente as correções certas sejam umas 10 vezes maiores pois a energia de ligação melhor convergida, por exemplo, tem essa proporção com a correção no nível MP2. 


\subsection{Conclusões}

Neste capítulo foi apresentada a aproximação harmônica para para modelar a superficie de energia potencial das moléculas e estimar, a primeira ordem perturbativa, dos fatores de Frack-Condon para uma molécula onde o efeito de correlação pósitron-elétron é vista, no sentido Born-Oppenheimer, como um potencial perturbativo na base do Hamiltoniano da molécula isolada. Foi encontrado que as correções de concavidade são as que, relativamente menores enquanto as mudanças na geometria de equilíbrio fazem os acoplamentos maiores com overtones. Existem termos no hamiltoniano do composto pósitron-molécula que fazem acoplamentos entre modos de vibração que podem ser grandes se as frequências dos modos acoplados são similares.

Utilizando derivadas numérica em base a os modos normais da molécula isolada, foram calculados termos de acoplamento utilizando diferencias finitas, para a molécula de HCN. Utilizando a teoria desenvolvida, foi encontrado que, uma vez o pósitron fica perto do átomo de Nitrogênio, o modo associado ao estiramento $\mathrm{C} \equiv \mathrm{N}$ é o que mais apresenta modificações. Por outro lado, qualitativamente, não existe acoplamento apreciável entre os modos longitudinais e transversais da molécula. Os dois modos longitudinais estão acoplados fracamente. O nível de cálculo empregado leva até correções pelo menos uma ordem de grandeza menor do que, possivelmente, seja as mais exatas e muito pequenas como para chegar até conclusões quantitativamente corretas. 


\section{Capítulo 8}

\section{Conclusões gerais e perspectivas}

\section{futuras.}

O trabalho desenvolvido permitiu discutir, a partir de cálculos teórico-computacionais efeitos não estudados previamente na literatura que podem ser tidos em conta ao modelar a aniquilação de pósitrons em moléculas.

Na primeira parte do trabalho, foi apresentado um método analítico para o cálculo de larguras de acoplamento eletrônico do pósitron $\left(\Gamma_{\nu}^{e}\right)$ em transições Raman-ativas mediado pelo potencial regularizado do tipo $-\alpha / 2 r^{4}$. Uma vez que a amplitude de captura do pósitron $\left(A_{\nu}\right)$ é aproximadamente constante no intervalo de polarizabilidade e energia do pósitron incidente, a maior parte de variação de $\Gamma_{\nu}^{e}$ é dada pela amplitude de transição vibracional que foi aproximada à da molécula isolada $\alpha_{\nu}$. As propriedades do modelo são resumidas, finalmente, pela relação:

$$
\Gamma_{\nu}^{e}(\mathrm{meV})=4.88 \times k_{\nu}\left|\alpha_{\nu}\right|^{2}(\text { u.a. })
$$

onde observamos que, segundo a estimativa, a ordem de grandeza das larguras para modos Raman-ativos e para modos IR-ativos é semelhante variando desde centésimos de meV até alguns poucos meV mantendo a relação (5.1).

Também foi desenvolvido o cálculo de curvas de energia potencial (CEP), no sentido Born-Oppenheimer para os sistemas $\mathrm{LiH}$, e+LiH e $\mathrm{NaH}$ e $+\mathrm{NaH}$ nos níveis de cálculo MCD-CI e MP2. As CEP da molécula e do composto diferem de maneira importante ao considerar efeitos da correlação Elétron-pósitron dependentes da geometria. Tal correlação permite acoplar mais modos vibracionais com o canal de entrada. No nível MP2, existe um acoplamento apreciável do primeiro modo excitado da molécula de LIH com os 
primeiros 3 níveis vibracionais do e+LiH e no nível MRD-CI, um acoplamento apreciável com os 5 primeiros.

Finalmente, foram calculadas as superficies de energia potencial para o HCN apresentando um modelo simples para estender o formalismo teórico para sistemas de mais de

um modo normal. É possível fazer uma aproximação harmônica da SEP e aplicar a teoria de perturbações para calcular os acoplamento entre o canal de entrada e os níveis vibracioanis do composto. Utilizando o metodo de diferenças finitas para o acálculo dos elementos da matriz hessiana do composto foram calculados termos de acoplamento para a molécula de HCN. Com a teoria desenvolvida, foi encontrado que no nível MP2, o pósitron forma um estado muito desligado da molécula ficando perto do átomo de $\mathrm{Ni}$ trogênio. Dessa maneira, o modo associado ao estiramento $\mathrm{C} \equiv \mathrm{N}$ é o que mais apresenta modificações. Por outro lado, qualitativamente, não existe acoplamento apreciável entre os modos longitudinais e transversais da molécula. Os dois modos longitudinais estão acoplados fracamente. O nível de cálculo empregado leva até correções pelo menos uma ordem de grandeza menor do que, possivelmente, seja as mais exatas e muito pequenas como para chegar até conclusões quantitativamente corretas.

\subsection{Perspectivas}

O resultado mais importante a curto prazo corresponde a o cálculo das larguras de acoplamento do pósitron em transições Raman-Ativas. Espera-se poder publicar, os resultados obtidos uma vez que são importantes para o entendimento do processo. É preciso, no entanto, calcular as larguras para moleculas mais complexas e com valores de polarizabilidade diferentes uma vez que as moléculas apresentadas em este trabalho corresponden ao intervalo de energia de polarizabilidade menor a $10 \AA^{3}$. Esperamos que a equação (8.1) continue sendo útil para otras moléculas diferentes a os alcanos escolhidos.

Os cálculos dos efeitos da correlação elétron-pósitron no sentido Born-Oppenheimer no movimento dos núcleos, foi importante uma vez que corresponde ao primeiro passo para estimativas mais sofisticadas. Qualitativamente os resultados obtidos parecem validar a hipótese de que os efeitos de segunda ordem na correlação elétron-pósitron são importantes. No entanto, os níveis de cálculo empregados foram insuficientes para que esses efeitos de segunda ordem sejam comparaveis com os experimentos. É possível implementar algoritmos mais fortes como o método CI implementado no pacote GAMESS ou melhorar as bases da maneira que o grupo de professor Andrés Reyes propôs. 


\section{Apêndice A}

\section{Cálculo de estrutura eletrônica}

O cálculo das autofunções da equação de Schrödiger para $H_{\text {ele, definida pela equação }}$ (3.2), corresponde a uns dos problemas principais da química quântica. Neste apêndice, será apresentada, superficialmente, a base teórica dos métodos utilizados para lidar com o problema do cálculo da estrutura eletrônica e que foram referenciados no trabalho todo.

\section{A.1 Método Hartree-Fock}

A principal complicação que tem o problema eletrônico é a presencia, no hamiltoniano, do potencial de interação elétron-elétron. A ideia central, é supor que a dinâmica de cada elétron pode ser resolvida independentemente dos outros, por exemplo, tomando a interação de um elétron com os outros mediante um campo efetivo de maneira que o hamiltoniano seja separável em hamiltonianos independentes $h_{i}$

$$
H_{\text {ele }} \approx \sum_{i}^{n} h_{i}
$$

de maneira que a função de onda $\varphi(\mathbf{r} ; \mathbf{R})$ que mais se aproxima à solução verdadeira $\left(P h i_{0}\right)$ é o produto anti-simetrizado de funções de onda individuais.

$$
\varphi(\mathbf{r} ; \mathbf{R})=(n !)^{-1 / 2} \operatorname{det}\left|\phi_{a}(1) \phi_{b}(2) \cdots \phi_{z}(n)\right|
$$

onde, simplificando a notação, denotamos por $\phi_{a}(i)$ ao orbital (função de onda) ocupado pelo elétron $i$ com coordenada $\mathbf{r}_{i}$ e dependente, paramêtricamente da geometriada molécula $\mathbf{R}$, vezes a função de espin. Assim, $\phi_{a}$ que satisfaz

$$
h_{i} \phi_{a}(i)=E_{a} \phi_{a}(i)
$$


de maneira que $\phi_{u}$ com $u=1,2 \cdots, z$ forma um conjunto ortonormal e $u$ incorpora o estado de espin e o espacial.

Na aproximação de Hartree-Fock, especificamente, cada elétron é considerado movimentandose num campo eletrostático dos núcleos e no campo médio dos outros $n-1$ elétrons. Para obter a função de onda mais ajustada, usa-se o método variacional que envolve a minimização do funcional:

$$
\varepsilon(\mathbf{R})=\frac{\int \varphi(\mathbf{x} ; \mathbf{R}) H \varphi(\mathbf{x} ; \mathbf{R}) d r}{\int \varphi^{*}(\mathbf{x} ; \mathbf{R}) \varphi(\mathbf{x} ; \mathbf{R}) d \mathbf{x}}
$$

com a restrição de que os espin-orbitais sejam ortonormais. Acima, $\mathbf{x}$ faz referência às coordenadas espaciais $\mathbf{r}_{i}$ e de espin $\sigma_{i}$.

Fazendo os procedimentos de minimização, obtemos, para cada espin-orbital $\varphi_{\nu}$, as chamadas equações de Hartee-Fock. Para, especificamente o elétron 1, a sua equação de Hartree-Fock correspondente é:

$$
f_{1} \phi_{\nu}(1)=\epsilon_{\nu} \phi_{\nu}(1)
$$

onde $\varepsilon_{a}$ e o autovalor do operador $f_{1}$ é chamado operador de Fock:

$$
f_{1}=h_{1}+\sum_{u} J_{u}(1)-K_{u}(1)
$$

aqui, $h_{1}$ o hamiltoniano de interação dos núcleos com o elétron 1 e os operadores $J_{u}$ (de Coulomb) e $K_{u}$ (de troca) são definidos:

$$
\begin{aligned}
J_{u}(1) \phi_{\nu}(1) & =e^{2}\left[\int \phi_{u}^{*}(2) \frac{1}{r_{12}} \phi_{u}(2) d \mathbf{x}_{2}\right] \phi_{\nu}(1) \\
K_{u}(1) \phi_{\nu}(1) & =e^{2}\left[\int \phi_{u}^{*}(2) \frac{1}{r_{12}} \phi_{\nu}(2) d \mathbf{x}_{2}\right] \phi_{u}(1)
\end{aligned}
$$

de maneira que partindo dos autoestado $\left(\varepsilon_{\nu}\right)$ do operador $f_{1}$, o funcional (A.4) pode ser escrito da maneira $\varepsilon=\sum_{\nu} \epsilon_{\nu}$. Uma vez que o operador $f_{1}$ depende espin-orbitais de todos os elétrons da molécula, a solução é obtida de uma maneira auto-consistente. Chutando uma base para construir o operador de Fock, são resolvidas as equações de HF obtendo um novo conjunto de espin-orbitais e assim sucessivamente até alcançar um critério de convergência.

Uma vez determinados os orbitais que satisfazem as equações de HF num intervalo de convergência, o operador $f_{1}$ o operador fica bem definido de maneira que pode ser encontrado seu espectro dando a posibilidade de calcular mais espin-orbitais do que elétrons. Calculando $m$ espin-orbitais e arranjando eles em ordem crescente de seu autovalor $\varepsilon_{\nu}$ 
(entendido como a sua energia), os $n$ orbitais com mais baixa energia são chamados orbitais ocupados e aqueles $m-n$ não ocupados são chamados, usualmente orbitais virtuais. Assim, o funcional de energia $\varepsilon$ do estado base satisfaz $\varepsilon=\sum_{\nu} \varepsilon_{\nu}$.

No nivel Hartree-Fock restrito (RHF), no determinante (A.2), cada parte espacial de um espin-orbital é forçada a ser compartida por dois elétrons com espins opostos. Assim, a componente espacial do orbital é idêntica para esse par de elétrons sendo somente é necessário o calculo de $n / 2$ ou $(n+1) / 2$ funções espaciais dependendo se $n$ é par ou impar. Infelizmente, orbitais ocupados com a mesma parte espacial e diferente espin não tem interação de troca $J_{\nu}-K_{\nu}$ de maneira que o método perde acurácia. No método de Hartree-Fock não restrito (UHF) a função espacial de um par elétrons com espins opostos não é forçada a ser a mesma de maneira que existe interação de troca entre todos os orbitais obtendo energias de estado base menores. No entanto, a função resultante não é autoestado do operador $S^{2}$ de maneira que um espin total não é definido.

\section{A.1.1 Funções base}

A quantidade de rodadas necessárias para obter a convergência no método HF pode ser diminuída se os orbitais são expressos como uma combinação linear de funções de onda $\theta_{i}(k)$ cujas integrais do tipo (A.7) são fáceis de calcular.

$$
\phi_{i}=\sum_{j} c_{i j} \theta_{j}
$$

com $c_{i j}$ coeficientes desconhecidos. Dessa maneira, a equação (A.5) vezes $\theta_{i}^{*}(1)$ e integrada em $\left(r_{1}\right)$ fica:

$$
\sum_{j} F_{i j} c_{j a}=\varepsilon_{a} \sum_{j} S_{i j} c_{j a}
$$

Onde $F_{i j}=\int \theta_{i}^{*}(1) f_{1} \theta_{j}(1) d r_{1}$ e $S_{i j}=\int \theta_{i}^{*}(1) \theta_{j}(1) d r_{1}$ matrizes não necessariamente diagonais. O sistema (A.9) é conhecido como equações de Roothaan e o método as vezes é chamado de Hartree-Fock-Roothman (HFR). Uma vez que $f_{1}$ depende das funções $\phi_{a}$, os elementos da matriz $F_{i j}$ podem ser escritos da maneira:

$$
\begin{aligned}
F_{i j}= & \int \theta_{i}^{*}(1) h_{1} \theta_{j}(1) d \mathbf{r}_{1}+2 e^{2} \sum_{u, l, m} c_{l u}^{*} c_{m u} \int \theta_{i}^{*}(1) \theta_{l}^{*}(2) \frac{1}{r_{12}} \theta_{m}(2) \theta_{j}(1) d r_{1} d r_{2} \\
& -e^{2} \sum_{u, l, m} c_{l u}^{*} c_{m u} \int \theta_{i}^{*}(1) \theta_{l}^{*}(2) \frac{1}{r_{12}} \theta_{j}(2) \theta_{m}(1) d r_{1} d r_{2}
\end{aligned}
$$

Que pode ser grandemente simplificada se é escolhida uma base $\theta_{i}$ adequada. 
Em principio, deve ser usado um conjunto completo de funções para representar exatamente os orbitais. No entanto, um conjunto infinito de funções não é computacionalmente viável e portanto sempre é usada uma base finita obtendo um erro devido a este truncamento. Dessa maneira, é uma consideração importante manter o numero de funções base pequeno enquanto o erro de truncamento é mantido baixo também.

Os orbitais tipo Slater (STOs) são as funções da maneira:

$$
R(r)=N r^{n-1} e^{-\zeta r}
$$

onde $n$ é um numero natural, $N$ é a constante de normalização, $r$ é a coordenada medida desde o núcleo atômico, $\zeta$ um exponente orbital que usualmente é obtido com um ajuste dos orbitais atômicos.

Os Orbitais tipo Gaussiana (GTOs) introduzidos por S.F. Boys em 1950 [? ] permitiram que a complexidade das equações de Roothaan, para moléculas, seja baixada até um nível tratável. Os GTO são funções da forma:

$$
\theta_{i j k}\left(\mathbf{r}_{1}-\mathbf{r}_{0}\right)=\left(x-x_{0}\right)^{i}\left(y-y_{0}\right)^{j}\left(z-z_{0}\right)^{k} e^{-\alpha\left|r-r_{0}\right|^{2}}
$$

onde $\left(x_{0}, y_{0}, z_{0}\right)$ são as coordenadas do centro da função gaussiana. $i, j$ e $k$ inteiros não negativos e $\alpha$ um expoente positivo. para $i=j=k=0$ a Gaussiana é tipo s; quando $i+j+k=1$ é uma gaussiana tipo p; quando $i+j+k=2$, então é tipo d.

A principal vantagem dos GTOs é que o produto de duas gaussianas centradas em diferentes posições é equivalente a uma gaussiana centrada num ponto entre elas. Portanto, uma integral de dois elétrons de três ou mais gaussianas pode ser reduzida a uma integral sobre dois centros. mesmo a dificuldade das integrais gaussianas é diminuído, são necessários mais GTOs para atingir uma precisão similar às funções de onda atómicas. Para aliviar esse problema, alguns GTOs são agrupados para formar as chamadas de funções gaussianas contraídas que são combinações lineares das originais funciones gaussianas, $g_{i}$, centradas no mesmo núcleo atómico:

$$
\chi_{j}=\sum_{i} d_{j i} g_{i}
$$

com os coeficientes conhecidos $d_{i j}$ fixos durante o cálculo. Dessa maneira, a função de onda é escrita da maneira:

$$
\psi_{i}=\sum_{j} c_{i j} \chi_{j}
$$

A definição dos $d_{j i}$ permite diminuir grandemente a quantidade de coeficientes $c_{i j}$ que devem ser calculados. A base minimal é aquela onde é escolhida uma gaussiana para 
cada camada de elétrons e portanto resulta muito inexata. a base double-zeta (DZ) são escolhidas dois funções bases. Numa base triple-zeta (TZ) são escolhidas três funções base para representar cada orbital.

Têm muitas maneiras de construir bases gaussianas contraídas. Uma aproximação é escolher N gaussianas primitivas ajustadas a STOs que tenham sido optimizados por médio de cálculos HF atómicos. Essa expansão é designada STO-NG. Uma escolha comum é $\mathrm{N}=3$, e portanto a base escolhida é designada por STO-3G.

Usualmente, desde uma base grande de gaussianas, são escolhidos os coeficientes de maneira que são representadas por una quantidade menor de gaussianas. Por exemplo, no conjunto de bases $3-21 \mathrm{G}$, uma gaussiana contraída composta de três primitivas é usada para representar cada orbital do caroço interno. Além disso, cada camada do caroço de valência é representado por dois funções, a primeira uma gaussiana contraída de dos primitivas e a segunda, uma primitiva simples (usualmente difusa). As primitivas são optimizadas num cálculo auto-consistente atómico e o conjunto contraído é usado em cálculos moleculares. A base 6-31G* começa com a base 6-31G e agrega funções de polarização na forma de seis funções tipo d para cada átomo diferente de H. Mais uma estrela, agrega uma função polarização de mais.[62]

Esse tipo de contrações é muito importante no momento de calcular pois o tempo de computo, no método de HF em geral, cresce como $M^{4}$ onde $M$ é o numero de funções base total. Se essa quantidade é diminuída à metade, o tempo de computo vai ser diminuído 16 vezes.

\section{A.2 Correlação eletrônica}

Uma vez que os operadores de Hartre-Fock modelam as interações de um elétron com os outros como uma média, ignora as interações instantâneas dos elétrons e os efeitos quânticos na distribuição dos elétrons. Tais deficiências e as outras estão incluídas na correlação.

\section{A.2.1 Funções de configuração de estado}

Se definimos o estado base do operador de Fock $\Phi_{0}$ da maneira:

$$
\Phi_{0}=(n !)^{-1 / 2} \operatorname{det}\left|\phi_{1} \cdots \phi_{i} \cdots \phi_{j} \cdots \phi_{n}\right|=\left\|\phi_{1} \cdots \phi_{i} \cdots \phi_{j} \cdots \phi_{n}\right\|
$$

Onde temos usado o mesmo numero para o elétron quanto para o orbital e mudado a notação $\|\cdots\|$ para designar implicitamente o determinante de Slater. Daqui, se 
conhecemos os orbitais virtuais ou seja aqueles normalmente vazios no estado base do operador de Fock, o método MP supõe que eles podem ser enchidos de maneira que podemos definir a primeira função excitada:

$$
\Phi_{i}^{l}=\left\|\phi_{1} \cdots \phi_{l} \cdots \phi_{j} \cdots \phi_{n}\right\|
$$

quanto a função duplamente excitada:

$$
\Phi_{i j}^{l m}=\left\|\phi_{1} \cdots \phi_{i} \cdots \phi_{m} \cdots \phi_{n}\right\|
$$

Da mesma forma, é possível construir outros determinantes multiplamente excitados. Cada um dos determinantes ou uma combinação linear de algum deles, incluindo simetrias eletrônicas, é chamado função de estado de configuração (CSF). Cada um deles pode ser usado para construir uma melhor representação do estado-base ou dos estados excitados.

\section{A.3 Teoria de Configuração de Interação (CI)}

A função de onda exata $\Psi$ para algum estado do sistema, pode ser escrita na forma:

$$
\Psi=C_{o} \Phi_{0}+\sum_{a, p} C_{a}^{p} \Phi_{a}^{p}+\sum_{\substack{a<b \\ p<q}} C_{a b}^{p q} \Phi_{a b}^{p q}+\sum_{\substack{a<b<c \\ p<q<r}} C_{a b c}^{p q r} \Phi_{a b c}^{p q r}+\cdots
$$

onde $C$ s são os coeficientes de expansão. A energia de correlação é basicamente a diferencia entre o limite $\mathrm{HF}$ e a energia exata. No entanto, dada a impossibilidade de lidar com uma base infinita de determinantes de Slater para $n$ elétrons, a quantidade de eles, é reduzida y dependendo da maneira na que é feita a redução, o método toma seu nome. Um nível full CI é aquele onde todas as CSFs da apropriada simetria, são usadas para um dado conjunto finito de bases. No entanto, a quantidade de determinantes possíveis para n elétrons usando $M$ bases, é dado pela sua combinatória. Embora, uma pequena quantidade de CSF pode dar conta de grandes correções.

A limitação mais simples é restringir a expansão a os determinantes simples e duplamente excitados denotada como SDCI. Se só são usados os determinantes duplamente excitados, o nível é chamado DCI. Dessa mesma maneira, se é incluída, a simples, dupla, tripla e quadrupla excitação além do estado base, é chamada SDTQCI. Como os determinantes quadruplamente excitados podem ser muito importantes no cálculo da energia de correlação, mas são muito pesados computacionalmente, a correção de Davidson foi 
proposta para estimar a contribuição dos determinantes quadruplamente excitados $\Delta E_{Q}$

$$
\Delta E_{Q}=\left(1-C_{0}^{2}\right)\left(E_{D C I}-E_{S C F}\right)
$$

onde a energia $E_{D C I}$ é a energia do estado base e $C_{0}$ é o coeficiente de $\Phi_{0}$, ambos obtidos no nível DCI. Enquanto $E_{S C F}$ é a energia de $\Phi_{0}$ no nível HF.

\section{A.3.1 Teoria de perturbações de Molle-Plesset}

Um dos métodos para lidar com o problema é o de perturbações de Molle-Plesset (MP). A ideia básica do método é partir do método Hartree-Fock definindo o operador Hamiltoniano de ordem zero $H^{(0)}$ como a suma dos operadores de Fock $F=\sum_{i} f_{i}$ definidos na equação (A.6) mais o valor esperado:

$$
H^{(0)}=F+\left\langle\Phi_{0}|(H-F)| \Phi_{0}\right\rangle
$$

onde H é o hamiltoniano exato e $\left|\Phi_{0}\right\rangle$ a função que satisfaz $F\left|\Phi_{0}\right\rangle=\varepsilon\left|\Phi_{0}\right\rangle$ é o estado base do operador de Hartree-Fock com $\varepsilon$ definida na equação (A.4). Enquanto o potencial perturbativo $(V)$ é chamado de potencial de correlação:

$$
V=H-\left(F+\left\langle\Phi_{0}|(H-F)| \Phi_{0}\right\rangle\right)
$$

De maneira que o Hamiltoniano total $(H)$ satisfaz $H=H^{(0)}+V$. Daqui, pode-se observar que:

$$
F\left|\Phi_{0}\right\rangle-\left\langle\Phi_{0}|F| \Phi_{0}\right\rangle\left|\Phi_{0}\right\rangle=0 \Longrightarrow H^{(0)}\left|\Phi_{0}\right\rangle=\left\langle\Phi_{0}|H| \Phi_{0}\right\rangle\left|\Phi_{0}\right\rangle
$$

portanto, seguindo a ideia do método de perturbações usual o de Rayleigh-Schrodinger para distingui-lo, a energia da ordem zero é simplesmente a energia de hartree-Fock:

$$
E_{M P}^{(0)}=E^{(0)}=\varepsilon=\left\langle\Phi_{0}|H| \Phi_{0}\right\rangle
$$

Enquanto que a contribuição de primeira ordem é

$$
E_{M P}^{(1)}=\left\langle\Phi_{0}|V| \Phi_{0}\right\rangle=0
$$

Dessa maneira, sendo $\left|\Phi^{j}\right\rangle$ qualquer determinante de Slater multi-excitado com autoenergia $\varepsilon_{j}^{(0)}$, a contribuição de segunda ordem à energia de Hatree-Fock, é:

$$
E_{M P}^{(2)}=\sum_{j \neq 0} \frac{\left\langle\Phi_{j}|V| \Phi_{0}\right\rangle\left\langle\Phi_{0}|V| \Phi_{j}\right\rangle}{\varepsilon-\varepsilon_{j}^{(0)}}
$$


O teorema de Brillouin[63], porém, mostra que o potencial de correlação não pode produzir excitações simples no estado fundamental $\Phi_{0}$ de maneira que as contribuições na equação (A.25) vão ser dadas pelas contribuições entre spin-orbitais com excitações duplas ou maiores. No caso de excitações duplas, obtemos:

$$
E_{M P}^{(2)}=\frac{1}{4} \sum_{a, b}^{\text {ocup }} \sum_{p, q}^{v i r} \frac{(a b \| p q)(p q \| a p)}{\varepsilon_{a}+\varepsilon_{b}-\varepsilon_{p}-\varepsilon_{q}}
$$

onde:

$$
\begin{aligned}
(a b \| p q)=\quad & e^{2} \int \phi_{a}^{*}(1) \phi_{b}^{*}(2) \frac{1}{r_{12}} \phi_{p}(1) \phi_{q}(2) d x_{1} d x_{2} \\
& -e^{2} \int \phi_{a}^{*}(1) \phi_{b}^{*}(2) \frac{1}{r_{12}} \phi_{q}(1) \phi_{p}(2) d x_{1} d x_{2}
\end{aligned}
$$

Com $\phi_{a}$ e $\phi_{b}$ sendo espin-orbitais ocupados e $\phi_{p}$ e $\phi_{q}$ espin-orbitais virtuais. É possível incluir ainda mais correções denotadas MP3 e MP4 pelo ordem de cálculo.

\section{A.4 Teoria do funcional densidade (DFT)}

Mesmo o métodos baseados no método de Hartree-Fock são amplamente usados atualmente, tem algumas limitações principalmente ao momento de lidar com computações de uma grande quantidade de elétrons. Dai surge o método da Teoria de funcional densidade (DFT) que não está baseado no conceito de orbitais moleculares mas no conceito de densidade de probabilidade tendo em conta a correlação enquanto a demanda computacional é relativamente menor do que os métodos baseados em HF.

A ideia básica da DFT é que a energia de um sistema pode ser escrita em termos da sua densidade eletrônica. Formalmente, a energia é tratada como um funcional no sentido de que para uma densidade de probabilidade $\rho(r)$, tem associada, a ela, somente uma energia.[64] Essa ideia não trivial só conseguiu ser provada até 1964 no trabalho de P. Hohenberg d W. Kohn [65]. Sem dar, no entanto, a forma dos funcionais. A forma explicita do funcional foi publica um ano depois por Kohn e Shan [66]. A energia do estado base eletrônico para um sistema de elétrons pode ser escrito da forma:

$$
\begin{aligned}
E[\rho]=\quad & -\frac{1}{2} \sum_{i=1}^{n} \int \psi_{i}^{*}\left(\mathbf{r}_{1}\right) \nabla_{i}^{2} \psi_{i}\left(\mathbf{r}_{1}\right) d \mathbf{r}_{1}-e^{2} \sum_{j=1}^{N} \frac{Z_{1}}{r_{j}} \rho\left(\mathbf{r}_{1}\right) d \mathbf{r}_{1} \\
& +\frac{e^{2}}{2} \int \frac{\rho\left(\mathbf{r}_{1}\right) \rho\left(\mathbf{r}_{2}\right)}{r_{12}} d \mathbf{r}_{1} d \mathbf{r}_{2}+E_{x c}[\rho]
\end{aligned}
$$


onde os orbitais $\psi_{i}(i=1,2, \cdots, n)$ são os chamados orbitais de Kohn-Sham que são as soluções da equação dada acima. Alí, a densidade de probabilidade do estado base é dada por:

$$
\rho(\mathbf{r})=\sum_{i=1}^{n}\left|\psi_{i}(\mathbf{r})\right|^{2}
$$

O primeiro termo da equação (A.30) representa a energia cinética dos elétrons, o segundo, a atração elétron-núcleo onde a soma é feita sobre os $N$ núcleos com sub-índice $j$ e número atómico $Z_{j}$ enquanto que o terceiro termo representa a interação entre d densidade de probabilidade (soma de todos os orbitais KS) nas coordenadas $\mathbf{r}_{1}$ e $\mathbf{r}_{2}$ e o último é o a energia de correlação-troca que também é um funcional da densidade e leva em conta todas as interações eletrônicas não clássicas e que usualmente, é escolhido de maneira aproximada.

Os orbitais são obtidos ao resolver consistentemente a energia eletrônica $E[\rho]$ com a densidade dada pela equação (A.31) obtendo:

$$
\left\{-\frac{1}{2} \nabla_{1}^{2}-e^{2} \sum_{j=1}^{N} \frac{Z_{j}}{r_{j 1}}+e^{2} \int \frac{\rho\left(\mathbf{r}_{2}\right)}{r_{12}} d \mathbf{r}_{2}+V_{X C}\right\} \psi_{i}\left(\mathbf{r}_{1}\right)=\varepsilon_{i} \psi_{i}\left(\mathbf{r}_{1}\right)
$$

onde $\varepsilon_{i}$ são as energia dos orbitais KS e o potencial de troca-correlação, $V_{x c}$ é a derivada funcional da energia de troca-correlação:

$$
V_{x c}[\rho]=\frac{\delta E_{x c}[\rho]}{\delta \rho}
$$

As equações KS são resolvidas de maneira autoconsistente usualmente numérica mente ou desde uma densidade de probabilidade obtida, tipicamente, usando a superposição das densidades atômicas que podem ser resolvidas, igualmente ao método HF, usando funções base adequadas.

\section{A.4.1 Funcionais troca-correlação}

Têm numerosos métodos que têm sido desenvolvidos para obter formas aproximadas para a energia de troca-correlação; a busca de métodos mais apropriados é uma área ativa nos esforços atuais. Num deles, na aproximação de densidade local (LDA), o funcional de troca-correlação é

$$
E_{x c}=\int \rho(\mathbf{r}) \varepsilon_{x c}[\rho(\mathbf{r})] d \mathbf{r}
$$

onde $\varepsilon_{x c}[\rho(\mathbf{r})]$ é a energia de troca-correlação por elétron num gás de homogêneo com densidade constante que supreendentemente é muito precisa. Para dar conta da inhomogeneidade da densidade eletrônica, tem sido proposta uma grande quantidade de funcionais corregidos com nomes como: nPWPW91, B3LYP, MPW1K, PBE1PBE, BLYP, 
BP91 e PBE. Por exemplo, o funcional BLYP faz uma mistura entre o funcional de troca de Becke[67] e o funcional de correlação de Lee-Yang-Parr[68]. O funcional B3LYP representa cálculos "hibridos"de DFT que usam correções baseadas em Hartree-Fock. 


\section{Apêndice B}

\section{Aspectos teóricos da espectrometria vibracional}

Neste capítulo serão apresentados os cálculos feitos para obter alguns resultados incumbentes à dinâmica vibracional da molécula.

\section{B.1 Análise vibracional}

Uma vez definida a matriz Hessiana da maneira feita na equação (3.6), usualmente é redefinida em coordenadas ponderadas pela massa:

$$
f_{q_{i} q_{j}}=\left(\frac{\partial^{2} V}{\partial q_{i} \partial q_{j}}\right)_{0}
$$

com $q_{i}$ definida pela equação (3.7). A diagonalização da matriz Hessiana define os modos normais e as frequências de ressonância da maneira exposta na equação (3.9) no entanto, existem autovalores nulos que correspondem a translações e rotações. Computacionalmente, nõ são exatamente zero mas muito pequenos. A maneira mais rigorosa de encontrar e remover essas coordenadas é apresentada a continuação.

\section{B.1.1 Separação de vibrações e rotações.}

Esse tipo de condições também conhecidas como condições de Eckart, procuram separar os movimentos externos como translações e rotações, dos movimentos internos como as vibrações. Rigorosamente, os movimentos não são separáveis exatamente mas é uma aproximação razoável para números quânticos pequenos. As condições fazem referência a o conjunto de translações atômicas que definem o sistema de coordenadas rotativo de 
maneira que é possível tirar, na equação os autovalores correspondentes a translações e rotações.

As primeiras três condições para definir nosso sistema é que o origem do sistema de coordenadas corresponda às coordenadas do centro de massa $(\mathbf{R})$, ou seja que se as posições dos átomos com massa $m \alpha$ é dada pelos vetores $\mathbf{r}_{\alpha}$

$$
\mathbf{R}=\frac{\sum_{\alpha} m_{\alpha} \mathbf{r}_{\alpha}}{\sum_{\alpha} m_{\alpha}}=0
$$

válidas para cada uma das três componentes do vetor. Se esse não fosse o caso, simplesmente é feita a transformação $\mathbf{r}_{\alpha}=\mathbf{r}_{\alpha}-\mathbf{R}$.

Dessa maneira, é possível obter os três vetores que produzem as translações do sistema que são:

$$
\mathbf{D}_{\alpha}=\sum_{\alpha} \sqrt{m_{\alpha}} \mathbf{q}_{\alpha}=\sum_{\alpha} m_{\alpha} \hat{\mathbf{e}}_{\alpha}
$$

onde $\mathbf{q}_{\alpha}$ são as coordenadas ponderadas pela massa enquanto $\hat{\mathbf{e}}_{\alpha}$ é o vetor normal da coordenada cartesiana $\alpha=x, y, z$

As outras três condições são obtidas de maneira que a molécula não faça rotações em relação a o sistema de referência. A condição mais natural é que o momento angular total da molécula, no sistema de referencia seja zero. Ou seja:

$$
\sum_{\alpha} m_{\alpha} \mathbf{r}_{\alpha} \times \mathbf{v}_{\alpha}=0
$$

onde $\mathbf{v}_{\alpha}$ é a velocidade do átomo $\alpha$. De maneira mais geral, se e feita um deslocamento $\delta \mathbf{r}_{\alpha}$ sem importar o tempo, a expressão (B.4) é equivalente a:

$$
\sum_{\alpha} m_{\alpha} \mathbf{r}_{\alpha} \times \delta \mathbf{r}_{\alpha}=0
$$

Para satisfazer essas condições é preciso encontrar a transformação que diagonalize o tensor de momento de inercia de maneira que os movimentos que são rotações ou translações, são retirados.

$$
\mathbf{I}=\left(\begin{array}{ccc}
I_{x x} & I_{x y} & I_{x z} \\
I_{y x} & I_{y y} & I_{y z} \\
I_{z x} & I_{z y} & I_{z z}
\end{array}\right)=\sum_{\alpha}\left(\begin{array}{ccc}
m_{\alpha}\left(y_{\alpha}^{2}+z_{\alpha}^{2}\right) & -m_{\alpha} x_{\alpha} y_{\alpha} & -m_{\alpha} x_{\alpha} z_{\alpha} \\
-m_{\alpha} x_{\alpha} y_{\alpha} & m_{\alpha}\left(x_{\alpha}^{2}+z_{\alpha}^{2}\right) & -m_{\alpha} y_{\alpha} y_{\alpha} \\
-m_{\alpha} x_{\alpha} z_{\alpha} & -m_{\alpha} z_{\alpha} y_{\alpha} & m_{\alpha}\left(x_{\alpha}^{2}+x_{\alpha}^{2}\right)
\end{array}\right)
$$

Com a matriz $\mathbf{X}$ que diagonaliza $\mathbf{I}$, feita com os autovalores normalizados dela. Esses auto valores vão ser usados para definir as rotações infinitesimais da molécula, de maneira 
que os vetores que geram rotações no sistema de coordenadas são:

$$
\begin{aligned}
& \mathbf{D}_{4, \alpha, i}=\left(\left(P_{y}\right)_{i} X_{\alpha, 3}-\left(P_{z}\right)_{i} X_{\alpha, 2}\right) / \sqrt{m_{i}} \\
& \mathbf{D}_{5, \alpha, i}=\left(\left(P_{z}\right)_{i} X_{\alpha, 1}-\left(P_{x}\right)_{i} X_{\alpha, 3}\right) / \sqrt{m_{i}} \\
& \mathbf{D}_{4, \alpha, i}=\left(\left(P_{x}\right)_{i} X_{\alpha, 2}-\left(P_{y}\right)_{i} X_{\alpha, 1}\right) / \sqrt{m_{i}}
\end{aligned}
$$

onde $\alpha=x, y, z ; i$ é sob todos os átomos e $P_{\alpha}=\left(\mathbf{r}_{i} \cdot X_{\alpha}\right)$ onde $\mathbf{r}_{i}$ é o vetor de posição da partícula $i$ medida desde o centro de massa.

Os vetores D deveram ser normalizados. Posteriormente, o processo de ortogonalização de Schmidt é usado para gerar os $N_{v i b}=3 N-6$ vetores que faltam os quais são ortogonais aos cinco ou seis vetores translacionais e vibracionais. Com esses vetores, é definida a matriz que transforma as coordenadas ponderadas pela massa $q$ até as coordenadas internas $S=D q$ ode foi feita a separação das vibrações e as translações.

\section{B.1.2 Cálculo de auto-valores vibracionais}

Se é calculada a matriz Hessiana em coordenadas ponderadas pela massa $H_{c p m}$, é possível transformar ela usando a matriz $D$ obtendo a matriz de força em coordenadas internas.

$$
f_{\text {int }}=D^{\dagger} f_{c p m} D
$$

De maneira que os auto-valores $\lambda=\omega^{2}$ da sub-matriz de $N_{v i b} \times N_{v i b}$ com autovetores $L$ que diagonalizam ela:

$$
L^{\dagger} f_{\text {int }} L=\Lambda
$$

onde $\Lambda$ é a matriz diagonal com auto valores $\lambda_{i}$

\section{B.1.3 Deslocamentos cartesianos e massa reduzida}

Usualmente, a matriz $\mathbf{l}=\mathbf{D L}$ que diagonaliza a hessiana $\left(f_{\text {cpm }}\right)$ em coordenadas ponderadas pela massa:

$$
L^{\dagger} D^{\dagger} f_{c p m} D L=l^{\dagger} f_{c p m} l=\Lambda
$$

é muito utilizada no análise vibracional. Especificamente os vetores coluna da matriz re-transformada $I_{\text {cart }}=M D L$ onde os elementos da matriz diagonal $M$ são definido por $M_{\alpha \alpha}=1 / \sqrt{m_{\alpha}}$ onde $\alpha$ inclui as coordenadas cartesianas de todos os átomos. portanto, especificamente:

$$
l_{c a r t k, i}=\sum_{j}\left(\frac{D_{k, j} L_{j, i}}{\sqrt{m_{k}}}\right)
$$


cujos vetores colunas são os modos normais em coordenadas cartesianas. O inverso de sua normalização ao quadrado corresponde á massa reduzida $\mu_{i}$ do modo vibracional $i$ :

$$
\mu_{i}=\left(\sum_{k} l_{c a r t k, i}^{2}\right)^{-1}
$$

\section{B.2 Potencial de Morse}

O potencial de Morse, é um modelo muito conveniente para a molécula diatômica. É muito melhor aproximação do que a aproximação harmônica porque explicitamente inclui efeitos de quebra do enlace e também a anarmonicidade dos enlaces reais. O potencial é descrito pela função:

$$
V(r)=D_{0}\left(1-e^{-a\left(r-r_{0}\right)}\right)^{2}+V(0)
$$

Onde $r$ é o comprimento entre os átomos, $r_{0}$ é a distancia de equilíbrio, $D_{0}$ é a profundidade do potencial (relativa a energia de dissociação dos átomos) e a controla a largura do potencial, $v(0)$ é a energia do mínimo.

O potencial além de dois das auto-funções são mostrada na figura B.1

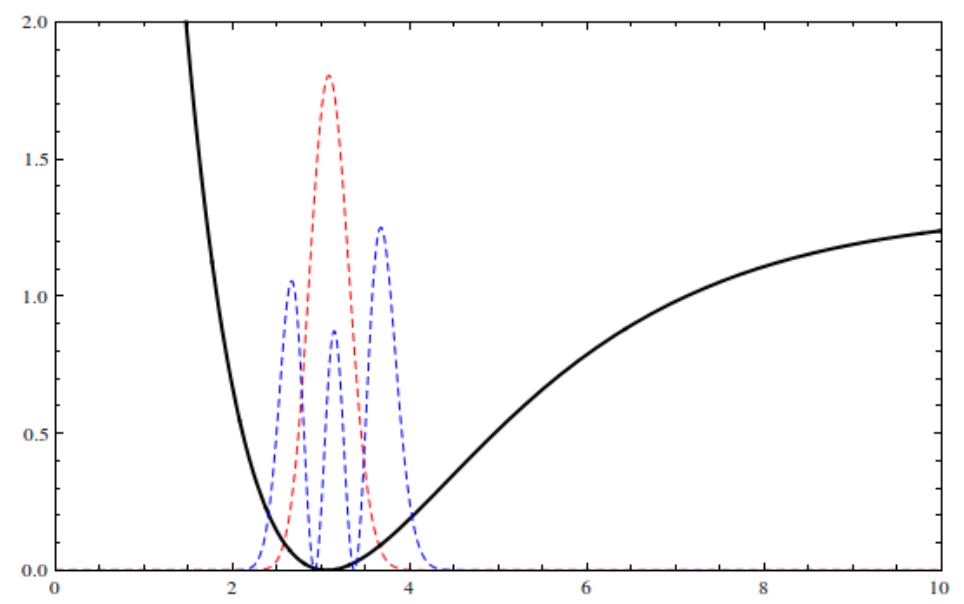

Figura B.1: Potencial de Morse (unidades arbitrarias) e as densidades de probabilidade de suas auto-funções $\Phi_{0}$ (vermelho) e $\Phi_{3}$ (azul)

Introduzindo a mudança de variáveis:

$$
\lambda=\frac{\sqrt{2 m D_{0}}}{a} \quad x=a r \quad x_{0}=a r_{0},
$$


os auto-estados e auto-energias podem ser escritas como:

$$
\begin{aligned}
\varepsilon_{n} & =\left(\lambda-n-\frac{1}{2}\right) \\
\Psi_{n}(z) & =N_{n} z^{\lambda-n-\frac{1}{2}} e^{-\frac{1}{2} z} L_{n}^{2 \lambda-2 n-1}(z)
\end{aligned}
$$

onde, $z=2 \lambda e^{-\left(x-x_{0}\right)} ; N_{n}=n !\left[\frac{(2 \lambda-2 n-1)}{\Gamma(n+1) \Gamma(2 \lambda-n)}\right]^{\frac{1}{2}}$ e $L_{n}^{\alpha}(z)$ é o polinômio de Laguerre:

$$
L_{n}^{\alpha}(z)=\frac{z^{-\alpha} e^{z}}{n !} \frac{d^{n}}{d z^{n}}\left(z^{n+\alpha} e^{-z}\right)
$$

As auto-energias tem a forma:

$$
E(\nu)=\omega_{0}(\nu+1 / 2)-\frac{\left[\omega_{0}(\nu+1 / 2)\right]^{2}}{4 D_{0}}
$$

onde $\nu$ é o numero quântico vibracional, e $\omega_{0}$ têm unidades de frequência. Ela, está relacionada com a massa reduzida $(\mu)$ da molécula e as constantes do potencial pela equação:

$$
\omega_{0}=a \sqrt{\frac{2 D_{0}}{m}}
$$

As auto-energias (B.17) em relação às energias do oscilador harmônico têm um termo adicional usualmente chamado termo de anarmónicidade

$$
\chi=\frac{\left[\omega_{0}(\nu+1 / 2)\right]^{2}}{4 D_{0}}
$$

sendo especialmente importante para níveis maiores, de maneira que existe um número quântico máximo

$$
\nu_{\max }=\frac{2 D_{0}-\omega_{0}}{\omega_{0}}
$$

a partir do qual, já não são permitidos mais níveis de energia e a molécula se dissocia.

\section{B.3 Coeficiente de Einstein}

Nesta seção vai ser intorduzida o coeficiente de Einstein empregado na seguinte seção. Considerando uma molécula exposta a luz com o vetor de campo elétrico $(\boldsymbol{\xi})$ na direção $z$ e oscilando com frequência $\omega$. O sistema pode ser modelado se o campo é descrito pela perturbação:

$$
V(t)=-\boldsymbol{\mu} \cdot \boldsymbol{\xi} \quad \boldsymbol{\xi}(t)=R e\left(\xi e^{-i \omega t}\right) \hat{\mathbf{e}}_{z}
$$


Utilizando a regra de ouro de Fermi, a taxa de transição desde o estado vibracional inicial $|i\rangle$ até o estado final $|f\rangle$ devido à perturbação é dado por:

$$
W_{i \rightarrow f}=\frac{2 \pi}{\hbar^{2}}\left|\mu_{z, f i}\right|^{2} \xi^{2} \rho\left(\omega_{i f}\right) \cos ^{2}(\theta)
$$

onde $\rho(\xi)$ é a densidade de radiação igual ao numero de fótons com energia $\hbar \omega_{f i}$ por unidade de volume e $\theta$ é o angulo entre os vetores $\boldsymbol{\xi}$ e $\boldsymbol{\mu}$. Depois de fazer a média angular:

$$
\left\langle\cos ^{2} \theta\right\rangle=\frac{1}{4 \pi} \int_{0}^{\pi} \int_{0}^{2 \pi} \cos ^{2} \theta \sin \theta d \theta d \phi=\frac{1}{3}
$$

Obtemos, dividindo pela energia total do campo $E=2 \xi^{2}$ obtemos o chamado de coeficiente de absorção de Einstein

$$
B=\frac{W_{i \rightarrow f}}{\rho\left(\omega_{i f}\right)}=\frac{\pi\left|\mu_{i f}\right|^{2}}{3 \hbar^{2}}
$$

por unidade de energia.

\section{B.4 Intensidade de Absorção infravermelha}

Nessa seção, estabeleceremos, a relação entre o coeficiente de absorção integrada $(A)$ e o dipolo de momento $\boldsymbol{\mu}_{f i}$ apresentada na figura (3.11). Para isso, considerando um plano de área $S$ numa distancia $x$ com radiação incidente desde a esquerda. Todos os fótons dentro de um cumprimento $c \Delta t$, e portanto um volume $S c \Delta t$ vai passar a través do plano num intervalo de tempo $\Delta t$. SE a densidade de energia do campo é $U$, a energia eletromagnética total passando pelo plano nesse intervalo é $U S c \Delta t$. O fluxo de energia, $J$, é a energia por unidade de tempo por unidade de área, ou seja: $J=U S c \Delta t /(S \Delta t)=c U$. A densidade de energia no intervalo de energia $(\omega, \omega+d \omega)$ é $d U=\rho_{\text {rad }}(E) d \omega$ e portanto o fluxo de energia no mesmo intervalo é $d J=c \rho_{\text {rad }}(E) d \omega$ definindo a intensidade de radiação de maneira que satisfaz: $d J=I(\omega) d \omega$. portanto, $I=c \rho_{\text {rad }}$.

Considerando que a absorção ocorre dentro de uma laje de espessura $d l$. Seja $n(\omega) d \omega$ a quantidade de moléculas capazes de absorber um fóton no intervalo de frequência $(\omega, \omega+d \omega)$, de maneira que o numero total de moléculas é $N=\int n(\omega d \omega)$. A taxa à qual alguma molécula absorve um fóton é, segundo (B.24), $W=B \rho_{\text {rad }}(E),{ }^{1}$ e uma vez que cada fóton tem uma energia $\hbar \omega$, a taxa de mudança de energia é dada por

$$
\frac{d U}{d t}=-\hbar \omega W n(\omega) d \omega=-n(\omega) \hbar \omega B \rho_{\text {rad }}(E) d \omega
$$

\footnotetext{
${ }^{1}$ Aqui fazemos a aproximação que a transição inversa ou seja de modo excitado até estado base é menos provável pois a população do estado excitado é muito menor.
} 
A energia que entra na laje num ponto $x$ medido desde um extremo, durante o intervalo $d t$ é $J(x) S d t$ enquanto que a energia que sai da laje em $x+d l$ é $J(x+d l) S d t$. Por conservação da energia, a diferencia é a mudança de energia na laje:

$$
\frac{d(U S d l)}{d t}=J(x+d l) A-J(x) A
$$

Expressão que leva até:

$$
\frac{d U}{d t}=\frac{J(x+d l)-J(x)}{d l}=\frac{d J}{d l}=\frac{d I}{d l} d \omega
$$

A qual usando a equação (B.25) fica:

$$
d I=-n(\omega) \hbar \omega B \rho_{\text {rad }} d l=-\frac{n(\omega) \hbar \omega}{c} B I d l
$$

A redução na intensidade quando o feixe passa a traves da amostra de cumprimento $d l$ com uma concentração molar $[C]$, é:

$$
d I=-\varepsilon(\omega)[C] I d l
$$

onde $\varepsilon$ é o coeficiente de absorção molar. Uma comparação entre as duas expressões leva até:

$$
\frac{\varepsilon(\omega)}{(\omega)}=\frac{\hbar n(\omega) B}{c[C]}
$$

fazendo o produto $d \omega$ em os dois lados e integrando sobre todas as frequências leva até $N \hbar B / c[C]$ na direita mas $N=[C] N_{A}$ onde $N_{A}$ é a constante de Avogadro. Dessa maneira:

$$
\int \frac{\varepsilon(\omega)}{\omega}=\frac{B \hbar N_{A}}{c}
$$

Para bandas de absorção, tipicamente a largura da frequência é muito pequena e portanto pode considerar-se constante nesse intervalo e reconhecendo: $A=\int \varepsilon(\omega) d \omega$ segue que:

$$
A=\left(\frac{\hbar \omega_{f} i}{c}\right) N_{A} B
$$

A partir da equação (B.24), substituindo B, temos:

$$
A=\frac{\pi N_{A} \omega_{f i}}{3 \hbar c} \mu_{f i}^{2}
$$




\section{Apêndice C}

\section{Aspectos formais do modelo de Gribakin-lee}

\section{C.1 Aniquilação do pósitron}

No formalismo de Feshbach, temos suposto que no existe decaimento directo devido à aniquilação pósitron-elétron. Essa suposição é válida toda vez que o tempo do estado meta-estavel seja definido tempo médio de vida da ressonância $\left(1 / \Gamma_{\nu}^{e}\right)$ e não pelo tempo de vida média asociado à aniquilação $\left(1 / \Gamma^{a}\right)$. Isto é

$$
\Gamma^{a} \ll \Gamma_{\nu}^{e}
$$

No caso geral no qual a taxa de aniquilação direta é importante, é adicionado, no Hamiltoniano do sistema, o termo imaginario $i \Gamma^{a}$ de maneira que fica:

$$
H^{\prime}=H+i \Gamma^{a}
$$

de maneira que, o potencial $V_{\text {opt }}$ é modificado acrescentando o potencial $i \Gamma^{a}$ e assim, definindo a largura total $\Gamma_{\nu}=\Gamma^{a}+\Gamma_{\nu}^{e}$ equação 4.30 é modificada da maneira:

$$
Z_{\mathrm{eff}}=\frac{2 \pi}{k_{\nu}} \rho_{d} \sum_{\nu} \frac{g_{\nu} \Gamma_{\nu}^{e}}{\left(k^{2} / 2-\omega_{\nu}-\varepsilon_{b}\right)+\left(\Gamma_{\nu} / 4\right)}=\frac{2 \pi^{2}}{k_{\nu}} \rho_{d} \sum_{\nu} \frac{\Gamma_{\nu}^{e}}{\Gamma_{\nu} \pi} \frac{g_{\nu} \Gamma_{\nu}}{\left(k^{2} / 2-\omega_{\nu}-\varepsilon_{b}\right)+\left(\Gamma_{\nu} / 4\right)}
$$




\section{C.2 modelo de Gribakin-Lee}

O modelo de Gribakin-Lee considera que a variação na estrutura eletrônica e nos estados vibracionais do alvo é quase-desprezível e assim, as funções de onda dos núcleos são praticamente as mesmas da molécula isolada e simplesmente é feito um deslocamento da energia do estado base sem variação na forma da SEP.

Nesse regime de afinidade positrônica, foi proposto que a função de onda do pósitron ligado fosse muito difusa e tivesse comportamento tipo $\left\langle r \mid \phi_{c a p}^{p}\right\rangle=\phi_{0}(r)=A e^{-\gamma r} / r$ (com $A \simeq(\gamma / 2 \pi)^{1 / 2}, \gamma=1 / a$ o comprimento de espalhamento[14] e tivesse simetria angular para fazer os cálculos mais simples.

O modelo GL considera o acoplamento contínuo-discreto do pósitron, como é definido em (4.11), por médio de um potencial efetivo de momento dipolar $V_{c a p}(r)=V(r)=$ $\boldsymbol{\mu} \cdot \mathbf{r} / r^{3}$. Se o pósitron com momento $\mathbf{k}$ incide numa molécula no estado vibracional $\left\langle\mathbf{R} \mid \eta_{0}\right\rangle=\Phi_{0}(\mathbf{R})$ onde $\{\mathbf{R}\}$ representa as coordenadas nucleares. Se $k^{2} / 2 \approx \omega_{\nu}+\varepsilon_{0}$, o pósitron vai ser aprisionado na VFR e a molécula vai ser excitada até o estado vibracional $\left\langle\mathbf{R} \mid \eta_{\nu}\right\rangle=\Phi_{\nu}(\mathbf{R})$. Partindo da equação (4.14), a largura correspondente $\Gamma_{\nu}^{e}$ pode ser calculada desde:

$$
\Gamma_{\nu}^{e}=2 \pi \int\left|U_{\nu, \mathbf{k}}\right|^{2} \delta\left(k^{2} / 2-\omega_{\nu}-\epsilon_{0}\right) \frac{d^{3} \mathbf{k}}{(2 \pi)^{3}}
$$

onde o termo $U_{\nu, \mathbf{k}}$ tem a forma:

$$
\begin{aligned}
U_{\nu, \mathbf{k}} & =\int \phi_{0}(\mathbf{r}) \Phi_{\nu}^{*}(\mathbf{R}) \frac{\boldsymbol{\mu} \cdot \mathbf{r}}{r^{3}} e^{i \mathbf{k} \cdot \mathbf{r}} \Phi_{0}(\mathbf{R}) d \mathbf{r} d \mathbf{R} \\
& =\frac{4 \pi i}{3} \frac{\boldsymbol{\mu}_{\nu} \cdot \mathbf{k}}{\sqrt{2 \pi \gamma}} 2 F_{1}\left(\frac{1}{2}, 1 ; \frac{5}{2} ;-\frac{k^{2}}{\gamma^{2}}\right)
\end{aligned}
$$

onde $\mu_{\nu}$ é o operador dipolo para a molécula já estudado na equação (3.11), $\boldsymbol{\mu}_{\nu}=$ $\left\langle\eta_{\nu}|\boldsymbol{\mu}| \eta_{0}\right\rangle$ e ${ }_{2} F_{1}$ é a função hipergeométrica resultando:

$$
\Gamma_{\nu}^{e}=\frac{16 \omega_{\nu} \mu_{\nu}^{2}}{27} h(\xi)
$$

Onde $h(\xi)=\xi^{3 / 2}(1-\xi)^{-1 / 2}\left[{ }_{2} F_{1}\left(\frac{1}{2}, 1 ; \frac{5}{2} ;-\xi /(1-\xi)\right)\right]^{2}$ é uma função adimensional de $\xi=1+\varepsilon_{0} / \omega_{\nu}$ tal que $\xi(0)=\xi(1)=0$ e $h_{\max } \approx 0.75 \mathrm{em} \xi \approx 0.89$.

A partir da equação (C.7), a largura elástica de uma VRF de um pósitron acoplado a um modo vibracional ativo no infravermelho pode ser facilmente estimada conhecendo a sua frequência $\omega_{\nu}$ e a grandeza da transição dipolar $\mu_{\nu}$ conhecida de experimentos de absorção no infravermelho.[69] 
Usando a função de onda $\phi_{0}(r)=A e^{-\gamma r} / r$, pode-se calcular a densidade $\rho_{d}$ resultando uma função linear de $\gamma[39]$. Podendo ser estimada como:

$$
\rho_{d}=(F / 2 \pi) \gamma
$$

com $F=0.66$. Uma vez que $\gamma \propto\left|\varepsilon_{0}\right|^{1 / 2}$ [39], pode-se argumentar que existe uma fraca dependência, no modelo, de $Z_{\text {eff }}$ e $\sqrt{\varepsilon_{0}}$, dependência muito importante no momento de fazer cálculo para vários tipos de moléculas.

Uma estimativa das larguras características $\Gamma_{\nu}^{e}$ associadas cada transição vibracional $\omega_{\nu}$ para a molécula $\mathrm{CH}_{3} \mathrm{Cl}$ utilizando o modelo GL, pode-se ver a Tabela 4.1.

\begin{tabular}{cccc} 
Modo & simetria & $\omega_{\nu}(\mathrm{meV})$ & $\omega_{\nu} \mu_{\nu}^{2}$ (u.a) \\
\hline$\nu_{1}$ & $a_{1}$ & 363 & $4.87 \times 10^{-6}$ \\
$\nu_{2}$ & $a_{1}$ & 168 & $1.91 \times 10^{-6}$ \\
$\nu_{3}$ & $a_{1}$ & 91 & $6.52 \times 10^{-6}$ \\
$\nu_{4}$ & $\mathrm{e}$ & 373 & $1.34 \times 10^{-6}$ \\
$\nu_{5}$ & $\mathrm{e}$ & 180 & $1.74 \times 10^{-6}$ \\
$\nu_{6}$ & $\mathrm{e}$ & 126 & $5.66 \times 10^{-7}$ \\
\hline
\end{tabular}

TABela C.1: Modos vibracionais para a molécula $\mathrm{CH}_{3} \mathrm{Cl}$ e o fator de largura $\omega_{\nu} \mu_{\nu}^{2}$ que esta associado à largura eletrônica pela equação (C.7)

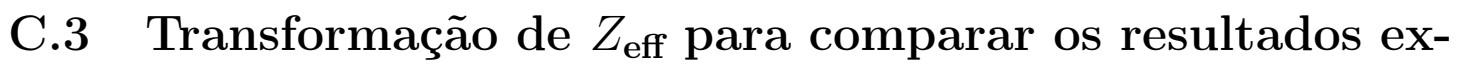 perimentais.}

A teoria de Gribakin-Lee, lida com feixes monocromáticos No entanto, os experimentos não lidaram com um feixe mono-energetico mas com um com certa distribuição térmica na energia dos pósitrons $(T=300 K)$. portanto, para fazer uma comparação de (C.3) e o experimento, (C.3) deve ser convoluído sobre a distribuição de energia do feixe. Isto último pode ser modelado por uma combinação da distribuição gaussiana na direção (z) e uma distribuição de Maxwell na direção transversal $\perp$. A densidade de probabilidade correspondente para a energia total do pósitron $\varepsilon=\varepsilon_{\perp}+\varepsilon_{z}$, é:

$$
f_{\epsilon}\left(\varepsilon_{\perp}, \varepsilon_{z}\right)=\frac{1}{k_{B} T_{\perp} \sqrt{2 \pi \sigma^{2}}} \exp \left[-\frac{\varepsilon_{\perp}}{k_{B} T_{\perp}}-\frac{\left(\varepsilon_{z}-\epsilon\right)^{2}}{2 \sigma^{2}}\right]
$$

Onde $k_{B}$ é a constante de Boltzmann, $T_{\perp}$ é uma temperatura efetiva transversal do feixe, $\epsilon$ é a energia média longitudinal dos pósitrons, como é medido no analisador e $\sigma=\delta_{z} / \sqrt{8 \ln 2}$ sendo $\delta_{z}$ a largura à meia altura. Os valores de $k_{B} T_{\perp}$ e de $\delta_{z}$ são tomados do experimento sendo $25 \mathrm{meV}$. Dado que $\delta_{z} \gg \Gamma_{\nu}$ para todos os $\nu$, a média $\bar{Z}_{\text {eff }}(\epsilon)=\int Z_{\text {eff }} f_{\epsilon}\left(\varepsilon_{\perp}, \varepsilon_{z}\right) d \varepsilon_{\perp} d \varepsilon_{z}$ pode ser feita analiticamente substituindo os perfiles de 
Breit-Wigner na equação (4.30) por funções $\delta$ de Dirac utilizando a seqüência de delta de Dirac:

$$
\delta(x)=\lim _{\varepsilon \rightarrow 0} \frac{1}{\pi} \frac{\varepsilon}{x^{2}+\varepsilon^{2}} .
$$

Dessa maneira, é obtido:

$$
\bar{Z}_{\mathrm{eff}}(\epsilon)=2 \pi^{2} \rho_{\mathrm{ep}} \sum_{\nu} \frac{g_{\nu} \Gamma_{\nu}^{e}}{k_{\nu} \Gamma_{\nu}} \Delta\left(\epsilon-\varepsilon_{\nu}\right)
$$

$\operatorname{com} \varepsilon_{\nu}=k^{2} / 2=\omega_{\nu}+\varepsilon_{0}$ a energia de ressonância e

$$
\Delta(E)=\frac{1}{2 k_{B} T_{\perp}} \exp \left[\frac{\sigma^{2}}{2\left(k_{B} T_{\perp}\right)^{2}} \exp \left(\frac{E}{k_{B} T_{\perp}}\right) \times\left(1+\Phi\left[\frac{E}{\sigma}+\frac{\sigma}{k_{B} T_{\perp}}\right]\right)\right]
$$

sendo $\Phi(x)$ a função erro. No limite $\Gamma^{a} \ll \Gamma_{\nu}^{e}$, é obtido $\Gamma_{\nu} \approx \Gamma_{\nu}^{e}$ de maneira que:

$$
\bar{Z}_{\mathrm{eff}}(\epsilon) \approx 2 \pi^{2} \rho_{\mathrm{ep}} \sum_{\nu} \frac{g_{\nu}}{k_{\nu}} \Delta\left(\epsilon-\varepsilon_{\nu}\right)
$$




\section{Bibliografia}

[1] Mohr, Peter J. Taylor, Barry N. Newell, David B. (2008). Rev. Mod. Phys. 80 (2): 633-730.

[2] Schultz, P. J., and K. G. Lynn, 1988, Rev. Mod. Phys. 60, 701.

[3] Puska, M. J., and R. M. Nieminen, 1994, Rev. Mod. Phys. 66,841.

[4] Dupasquier, A., and A. P. Mills, 1995, Eds., Positron Spectroscopy of Solids IOS, Amsterdam.

[5] Major, Z., S. B. Dugdale, R. J. Watts, G. Santi, M. A. Alam, S. M. Hayden, J. A. Duffy, J. W. Taylor, T. Jarlborg, E. Bruno, D. Benea, and H. Ebert, 2004, Phys. Rev. Lett. 92, 107003.

[6] Gidley, D. W., T. L. Dull, W. E. Frieze, J. Sun, A. F. Yee, C. V. Nguyen, and D. Y. Yoon, 2000, Appl. Phys. Lett. 76, 1282.

[7] David, A., G. Kogel, P. Sperr, and W. Triftshauser, 2001, Phys. Rev. Lett. 87, 067402 .

[8] Wahl, R. L., 2002 Principles an Practice of positron Emission Tomography (Lippincott, Williams and Wilkins, Philadelphia).

[9] G. F. Gribakin, J. A. Young, and C. M. Surko, Rev. Mod. Phys. 82, 2557 (2010)

[10] Dirac, P. A. M., 1930, Proc. Cambridge Philos. Soc. 26, 361.

[11] Pomeranchuk, I., 1949, Zh. Eksp. Teor. Fiz. 19, 183.

[12] C. M. Surko, G. F. Gribakin, and S. J. Buckman, J. Phys. B 38, R57 (2005).

[13] Surko, C. M., A. Passner, M. Leventhal, and F. J. Wysocki, 1988, Phys. Rev. Lett. $61,1831$.

[14] V. I. Goldanskii and Yu. S. Sayasov, Phys. Lett. 13, 300 (1964).

[15] Smith, P. M., and D. A. L. Paul, 1970, Can. J. Phys. 48, 2984. 
[16] Fraser, P. A., B. H. Bransden, P. G. Coleman, and W. Raith, 1982, Can. J. Phys. 60,565 .

[17] Gilbert, S. J., C. Kurz, R. G. Greaves, and C. M. Surko, 1997, Appl. Phys. Lett. $70,1944$.

[18] Gilbert, S. J., L. D. Barnes, J. P. Sullivan, and C. M. Surko, 2002, Phys. Rev. Lett. 88, 043201.

[19] M. T. do N. Varella, M. A. P. Lima, Phys. Rev. A 76, 052701 (2007).

[20] M. T. do N. Varella, E. M. de Oliveira e M. A. P. Lima, Nucl. Instrum. Meth. Phys. Res. B 266, 435 (2008).

[21] E. M. de Oliveira, M. A. P. Lima, S. d'A. Sanchez and M T. do N. Varella, Phys. Rev. 81, 012712 (2010).

[22] S. d'A. Sanchez, M. A. P. Lima, and M. T. do N. Varella, Phys. Rev. A 80, 052710 (2009).

[23] Sanchez, S. d'A., M. A. P. Lima and M. T do N. Varella, Phys. Rev. Lett.107, 103201 (2011).

[24] Heyland G R, Charlton M, Davies S A and Griffith T C 1986 Phys. Lett. A 119289

[25] Surko C M, Passner A, Leventhal M and Wysocki F J 1988 Phys. Rev. Lett. 611831

[26] U. Fano, Nueovo Cimento, 12, 156 (1935)

[27] Cohen-Tannoudji, Dupont-Roc, Grynberg. Atom-Photn Interactions: Basic processes and applications Wiley-VHC Verlag

[28] George B. Arfken. Mathematical methods for physicists

[29] L. D. Landau and E. M. Lifshitz, Quantum Mechanics (Pergamon, Oxford, 1977), 3rd ed

[30] E. Bright , J.C. Decius, P.C Cross Molecular vibrations Dover publications, New York (1955)

[31] Max Born; J. Robert Oppenheimer (1927). "Zur Quantentheorie der Molekeln". Annalen der Physik (in German) 389 (20): 457-484.

[32] A.C. Albrecht, J. Chem. Phys., 34, 1476 (1961).

[33] H.W. Schr0tter and H.W. K10ckner, in Raman Spectroscopy of Gases and Liquids, (A. Weber, ed.), Springer, Berlin, 1979, p. 123 
[34] J. Martin and S. Montero, J. Chem. Phys., 80, 4610 (1984).

[35] W. Domcke, J. Phys. B 14, 4889 (1981); Phys. Rep. 208, 97 (1991).

[36] H. Feshbach, Ann. Phys. N.Y. 5, 357 (1958); 19, 287 (1962).

[37] A. U. Hazi, T. N. Rescigno, and M. Kurilla, Phys. Rev. A 23, 1089 (1981).

[38] T. F. O’Malley, Phys. Rev. 150, 14 (1966).

[39] G. F. Gribakin and C. M. R. Lee, Phys. Rev. Lett. 97, 193201 (2006)

[40] Barnes, L. D., S. J. Gilbert, and C. M. Surko, 2003, Phys. Rev. A 67, 032706.

[41] Barnes, L. D., J. A. Young, and C. M. Surko, 2006, Phys. Rev. A 74, 012706.

[42] Young, J. A., and C. M. Surko, 2008c, Phys. Rev. A 78, 032702.

[43] J. R. Danielson, J. A. Young, and C. M. Surko, J. Phys. B 42, 235203 (2009).

[44] D. Assafrão and J. R. Mohallem, J. Phys. B 43, 155204 (2010).

[45] P. H. Amaral, and J. R. Mohallem, Phys. Rev. A 86, 042708 (2012).

[46] C. F. Gogtas, G. G. Balint-Kurti, and C. C. Marston, FGHEVEN, QCPE Program No. 647 (1993).

[47] C.C. Marston and G.G.Balint-Kurti, J.Chem. Phys.,91,3571 (1989).

[48] G. Reisfeld and U. Asaf, Phys. Rev. A 49, 348 (1994).

[49] Press, W. H.; Flannery, B. P.; Teukolsky, S. A.; and Vetterling, W. T. "Exponential Integrals." 6.3 in Numerical Recipes in FORTRAN: The Art of Scientific Computing, 2nd ed. Cambridge, England: Cambridge University Press, pp. 215-219, 1992.

[50] Principles and Applications of Positron and Positronium Chemistry; Jean, Y. C., Mallon, P. E., Schrader, D. M., Eds.; World Scientific: Singapore, 2003.

[51] S. Webb, T. Iordanov, S. Hammes-Schiffer, J. Chem. Phys. 2002, 117, 4106.

[52] S. P. Webb, T. Iordanov, and S. Hammes-Schiffer, J. Chem. Phys. 117, 4106-4118 (2002).

[53] Mitroy, J. Phys. ReV. A 2006, 73, 054502.

[54] Adamson P. E. et al. J. Phys. Chem. A, 112 (6), 1346

[55] de oliveira, Lima, D'a Sanchez Varella. Phys Rev A 81, 012712 (2010) 
[56] F.A. Gianturco, J. Franz, R.J.Buenker, H. Liebermann, L. Pichl, J. Rost, M. Tachikawa, M. Kimura.Phys Rev A 73, 022705 (2006)

[57] Franck, J. (1926). Elementary processes of photochemical reactions. Transactions of the Faraday Society 21: pp. 536-542.

[58] Y. Kita, R. Maezono, M. Tachikawa, M. Towler, R. Needs. J chem phys 131, 134310 (2009)

[59] A. C. L. Jones, J. R. Danielson, M. R. Natisin, and C. M. Surko Phys, Review Letters 110, 223201 (2013)

[60] Young, J. A., and C. M. Surko, 2007, Phys. Rev. Lett. 99, 133201.

[61] H. Chojnacki and K. Strasburger, Mol. Phys. 104, 2273 (2006).

[62] W. J. Hehre, L. Radom, P. v. R Schleyer, and J. A. Pople, Ab Initio molecular orbital theory Wiley, New York (1986)

[63] Szabo, Attila; Neil S. Ostlund (1996). Modern Quantum Chemistry. Mineola, New York: Dover Publications, Inc. pp. 350-353. ISBN 0-486-69186-1.

[64] S. Borman, Chem. Eng. News, 22, 68 (1990)

[65] P. Hohenberg, W. Khon, Phy. Rev 864 B136 (1964)

[66] W. Kohn and L. J. Sham, Phys. Rev., 1133, A140 (1965)

[67] A.D. Becke, J. Chem. Phys., 4524 (1986); Phys. REv. A, 3098,38 (1988)

[68] Lee, Yang, Parr, Phys. Rev. B, 785,37 (1988).

[69] L. M. Bishop and L. M. Cheung, J. Phys. Chem. Ref. Data 11, 119 (1982). 